2018-07-04

\title{
Modelling Critical Success Factors for Sustainability Initiatives in Supply Chains in Indian Context using Grey-DEMATEL
}

\section{KUMAR MANGLA, SACHIN}

http://hdl.handle.net/10026.1/11007

10.1080/09537287.2018.1448126

Production Planning \& Control (TPPC)

Taylor \& Francis

All content in PEARL is protected by copyright law. Author manuscripts are made available in accordance with publisher policies. Please cite only the published version using the details provided on the item record or document. In the absence of an open licence (e.g. Creative Commons), permissions for further reuse of content should be sought from the publisher or author. 


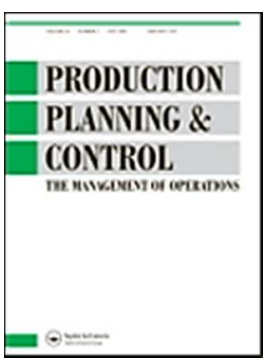

\section{Modelling Critical Success Factors for Sustainability Initiatives in Supply Chains in Indian Context using Grey- DEMATEL}

\begin{tabular}{|r|l|}
\hline Journal: & Production Planning \& Control \\
\hline Manuscript ID & TPPC-2017-0352.R3 \\
\hline Manuscript Type: & Research paper for Regular Issue \\
\hline Date Submitted by the Author: & 26-Feb-2018 \\
\hline Complete List of Authors: & $\begin{array}{l}\text { Luthra, Sunil; State Institute of Engineering and Technology (Formerly } \\
\text { known as Government Engineering College), Department of Mechanical } \\
\text { Engineering } \\
\text { Mangla, Sachin; University of Plymouth, Plymouth-PL4 8AA, United } \\
\text { Kingdom, Plymouth Business School } \\
\text { Shankar, Ravi; Indian Institute of Technology, Delhi , Department of } \\
\text { Management Studies } \\
\text { Prakash, Chandra; College of Management \& Economics Studies, UPES, } \\
\text { Dehradun } \\
\text { Jakhar, Suresh; Indian Institute of Management Lucknow }\end{array}$ \\
\hline Keywords: & $\begin{array}{l}\text { Critical Success Factors (CSFs), Grey-Decision Making Trial and Evaluation } \\
\text { Laboratory (DEMATEL), Sustainability Initiatives, Developing Country- } \\
\text { India, Supply Chain Management (SCM) }\end{array}$ \\
\hline \multicolumn{2}{|c}{} \\
\hline
\end{tabular}




\title{
Modelling Critical Success Factors for Sustainability Initiatives in Supply Chains in Indian Context using Grey-DEMATEL
}

\author{
Sunil Luthra* \\ Department of Mechanical Engineering, State Institute of Engineering and Technology (Formerly \\ known as Government Engineering College), Nilokheri-132117, Haryana, India \\ E-mail: sunilluthra1977@gmail.com \\ Phone: +91-9466594853

\section{Sachin Kumar Mangla} \\ Plymouth Business School, University of Plymouth, Plymouth-PL4 8AA, United Kingdom \\ E-mail: sachin.kumar@plymouth.ac.uk

\section{Ravi Shankar} \\ Department of Management Studies, Indian Institute of Technology, Delhi-110016, India \\ Email: ravi1@dms.iitd.ernet.in
}

\section{Chandra Prakash Garg}

Department of Transportation, School of Business

University of Petroleum and Energy Studies (UPES), Dehradun, India, 248007

E-mail - cpgarg86@gmail.com

\section{Suresh Jakhar}

Indian Institute of Management, Lucknow-226013, Uttar Pradesh, India Email: suresh87jakhar@gmail.com

*Corresponding Author 
Abstract: Sustainability assists organisations to attain competitive edges through enhanced ecological, financial and societal performances of their entire supply chains. The adoption of sustainability is usually difficult for the organisations, especially in a developing nation, such as India due to the existence of various significant factors related to finance, management, government regulations etc. The present paper uncovers the Critical Success Factors (CSFs) for effective adoption of sustainability initiatives in the supply chain in Indian context. Fifteen CSFs for the successful adoption of sustainability initiatives were identified and finalised firstly from the literature and followed by expert inputs. A methodology based on Grey-Decision Making Trial and Evaluation Laboratory (DEMATEL) was used to envisage the organisation of complex causal relationships between the recognised CSFs. "Government Legalisation" has been found to be the most influential factor and "Community Welfare and Development" is most easily influenced factor. A multiple case example of three automotive companies operating in India is conducted. This work proposes a decision framework to assist managers in revealing the interactive relations among sustainability oriented CSFs in the supply chain. To the end, some important policy measures and recommendations are proposed to help practicing managers and government bodies to adopt and effectively manage the concepts of sustainability oriented supply chains in India.

Keywords: Critical Success Factors; Grey-DEMATEL; Sustainability Initiatives; India; Automotive Sector; Supply Chain Management; Competitive Advantage.

\section{Introduction}

Currently, various stakeholders, such as investors, customers, regulatory bodies, non-government organisations and the community at large are enthusiastically examining industrial supply chains for their Critical Success Factors (CSFs) like cost, quality, delivery, emissions etc. (Klassen and Vereecke, 2012) and also their interdependency with an aim to develop approaches towards green and sustainable supply chain (Chen and Chai, 2010). Adding sustainability initiatives to supply chains are an effective tool for material management, information sharing and distribution, capital flow, and cooperation among supply chain members to enhance their triple bottom line performances (Seuring and Müller, 2008; Chaabane et al., 2011; Ageron et al., 2012; Gopalakrishnan et al., 2012; Seuring, 2013; Brandenburg et al., 2014). Sustainability initiatives are an excellent way to expand the accountability of supply chain members in reducing pollution 
and waste (Zailani et al., 2012; Marshall et al., 2015). The supply chain sustainability can be analysed from different perspectives, however, it is significant to evaluate the sustainability of supply chain from the system perspective (Ogunbiyi et al., 2014), which is being pursued in this work.

Over past few years, the developing and developed nations are focusing on sustainability targeted initiatives in supply chains to manage their economic, social and ecological issues. However, the concepts of sustainability initiatives in the supply chain in developing nations are immature in comparison to developed nations but growing up at fast pace (Turker and Altuntas, 2014; Silvestre, 2015). In case of developing nations like India, the industries have limited understanding on reducing their carbon emissions (Irani et al., 2017). In line with this, the implementation of sustainability initiatives in supply chains is also challenging in the developing nations, such as India (Al Zaabi et al., 2013).

The Indian automobile industry is growing very rapidly and involved in various functions of value chain, such as material procurement, production, marketing and distribution and has started to comprehend the significance of sustainability focused concepts in their supply chains (Luthra et al., 2015; India in Business, 2016). In addition to this, Indian automotive industry is committed to develop a sustainability culture into their business ecosystem. However, a very limited number of studies are available that evaluate causal relationships between the sustainability initiatives implementation CSFs. To deal with this, managers are required to recognise the critical factors, which may guide them towards the successful implementation of sustainability practices (Grimm et al., 2014).

In fixing the objectives of this research, we seek to keep the content of this study as generic as possible for a wider applicability.

The present research work has the following two objectives:

- To identify the CSFs to effective adoption of sustainability initiatives in supply chains;

- To uncover the causal relationships among the identified CSFs and to classify them into cause and effect for effective adoption of sustainability initiatives in supply chains.

As this paper aims to uncover the CSFs relevant to implementation of sustainability initiatives in industrial supply chains from the system perspective, a Grey based DEMATEL approach has been proposed to understand the structure of complicated causal relationships among the identified 
CSFs and to classify them into cause and effect groups. DEMATEL can extract the interrelationships as well as the intensity of interrelationship between various elements of a system (Hsu et al., 2013; Seleem et al., 2016). Grey set theory is an approach that can incorporate ambiguity and uncertainty into the analysis process. The Grey-DEMATEL method can effectively manage not only uncertain judgments and but also may flexibly deal with vagueness in evaluating cause and effect relations among factors (Bai and Sarkis, 2013). To reveal the applicability of the suggested grey based DEMATEL approach, a multiple case study of three Indian automotive companies is discussed.

The rest of the paper is structured as follows: A review of the literature related to this study is provided in Section 2. CSFs to supply chain sustainability are identified in Section 3. The framework proposed in this research is presented in Section 4. The research methodology is elucidated in Section 5. An application example and related results are presented in Section 6. Sensitivity analysis is conducted in Section 7. Discussion of the research findings and implications for managers are provided in Section 8. Lastly, the conclusions, limitations of the study and possibilities of future work are presented (see Section 9).

\section{Literature Review}

This section contains the relevant literature on supply chain management and sustainability, modelling approaches used in sustainability initiatives in supply chains, and describes the research gaps for the present study.

\subsection{Supply chain management and sustainability}

Environment degradation, global warming and ozone layer depletion have encouraged widespread concerns over sustainability issues in supply chain activities in recent years (Büyüközkan and Çifçi, 2011). Carter and Rogers (2008) stated sustainability in supply chain management as the systematic accomplishment of an organisation's economic, environmental and social goals through coordination and collaboration of key inter-organisational operations for humanising long-term economic, environmental and social performance of a firm along with all members of its supply chain. Based on extant literature, a brief review on sustainability in the supply chain is presented. Faisal (2010) put forward an approach towards an effective adoption of sustainable practices in a supply chain by considering the dynamics between various CSFs/enablers to 
develop a sustainable supply chain. Further, a hierarchy based structural model of the enablers of sustainability in the supply chain was also presented in this study. Al Zaabi et al. (2013) analysed the interaction between thirteen barriers to implement Sustainable Supply Chain Management (SSCM) practices by taking a case study in an Indian fastener industry. The findings suggest that three barriers (i.e. complex design, lack of clarity and the cost for environmentally friendly packaging) are critical barriers and require more focus than other barriers towards their removal. Diabat et al. (2014) analysed enablers for the adoption of sustainability in supply chains for Indian textile industries. The result discovers that five enablers (i.e. adoption of green practices, safety standards, community welfare, health and safety concerns, and employment stability) dominated the textile industry's sustainable supply chain practices.

Ageron et al. (2012) proposed and validated a framework by using the empirical study of selected French organisations. The findings provided a variety of future research directions in the emerging field of sustainable supply chain. Tseng and Hung (2014) formulated a decision model to evaluate carbon dioxide emissions and operational costs in the apparel manufacturing industry. The results suggested that the regulatory bodies force organisations to support for the social costs of carbon dioxide emissions and provided a helpful method to reduce carbon dioxide emissions.

Ahi and Searcy (2015) identified and analysed the metrics from previous published literature on green supply chain management (GSCM) and SSCM. Various unique metrics were identified in this study and the top five metrics were quality, air emissions, greenhouse gas emissions, and energy use and energy consumption. Taticchi et al. (2015) reviewed the existing literature published from 2000 to 2013 related to SSCM decision-support tools and the measurement of performance. The literature analysis suggested that it is significant to mix sustainability concepts for higher performance in business.

\subsection{Modelling approaches used in sustainability initiatives in supply chains}

Various researchers utilised different modelling techniques/methodologies by incorporating sustainability from a supply chain context. A brief review of various modelling techniques used in sustainability initiatives in the supply chain is given in Table 1.

Table 1: Modelling techniques incorporating sustainability in supply chain

\begin{tabular}{|c|c|c|c|}
\hline $\begin{array}{l}\text { S. } \\
\text { No. }\end{array}$ & Researcher (Year) & Modelling techniques used & Issues addressed \\
\hline 1 & Sarkis & Grey theory and Rough set & Sustainability focused supplier \\
\hline
\end{tabular}




\begin{tabular}{|c|c|c|c|}
\hline & $(2010)$ & & selection \\
\hline 2 & Faisal (2010) & Interpretive Structural Modelling (ISM) & Enablers of SSCM \\
\hline 3 & $\begin{array}{l}\text { Büyüközkan and } \\
\text { Çifçi (2011) }\end{array}$ & Fuzzy Analytical Hierarchical Process (FAHP) & $\begin{array}{l}\text { Sustainability focused supplier } \\
\text { selection }\end{array}$ \\
\hline 4 & $\begin{array}{l}\text { Amindoust et al. } \\
(2012)\end{array}$ & Fuzzy inference system & $\begin{array}{l}\text { Sustainability focused supplier } \\
\text { selection }\end{array}$ \\
\hline 5 & $\begin{array}{l}\text { Al Zaabi et al. } \\
(2013)\end{array}$ & ISM & Barriers to implement SSCM \\
\hline 6 & $\begin{array}{l}\text { Govindan et } \\
\text { al.(2013) }\end{array}$ & $\begin{array}{l}\text { Fuzzy TOPSIS (The Technique for Order of } \\
\text { Preference by Similarity to Ideal Solution) }\end{array}$ & $\begin{array}{l}\text { Sustainable supplier } \\
\text { performance measurement }\end{array}$ \\
\hline 7 & Mangla et al. (2013) & ISM & $\begin{array}{l}\text { Sustainability focused product } \\
\text { recovery systems }\end{array}$ \\
\hline 8 & $\begin{array}{l}\text { Bai and Sarkis } \\
(2014)\end{array}$ & Rough set theory and Data envelopment analysis & $\begin{array}{l}\text { Sustainable supplier } \\
\text { performance measurement }\end{array}$ \\
\hline 9 & Diabat et al. (2014) & ISM & Enablers of SSCM \\
\hline 10 & $\begin{array}{l}\text { Tseng and Hung } \\
(2014)\end{array}$ & Mixed integer programming & $\begin{array}{ll}\text { SSCM } & \text { performance } \\
\text { management } & \end{array}$ \\
\hline 11 & Azadi et al. (2015) & Fuzzy DEA (Data Envelopment Analysis) & $\begin{array}{l}\text { Sustainable supplier } \\
\text { performance evaluation }\end{array}$ \\
\hline 12 & Lin et al. (2015) & Analytical Network Process (ANP) & $\begin{array}{l}\text { Sustainability focused Supplier } \\
\text { selection }\end{array}$ \\
\hline 13 & Tseng et al. (2015) & Fuzzy Delphi Method (FDM) and ANP & $\begin{array}{l}\text { Sustainable supplier } \\
\text { performance measurement }\end{array}$ \\
\hline 14 & $\begin{array}{l}\text { Gopal and Thakkar } \\
\text { (2016a) }\end{array}$ & ISM & SSCM practices \\
\hline 15 & $\begin{array}{l}\text { Gopal and Thakkar } \\
(2016 \mathrm{~b})\end{array}$ & Structural Equation Modelling (SEM) & SSCM practices \\
\hline 16 & Su et al. (2016) & Grey based DEMATEL & Sustainability focused Supplier \\
\hline
\end{tabular}

According to Table 1, researchers have widely used modelling techniques, such as ISM, DEA, ANP, AHP, to analyse the sustainability related issues in a supply chain context. However, a limited application of grey based DEMATEL can be seen in the context of sustainable supply chains (Table 1). The reason behind this could be grey based DEMATEL technique is quite immature due to its limited applicability, but can provide superior outcomes as compared to ISM/AHP/ANP/DEA etc. (Bai and Sarkis, 2013). In this work, grey based DEMATEL is used for analysing the CSFs for sustainability in supply chains in a developing country context, specifically in India. The use of Grey-DEMATEL can be applied with limited data set along with focusing on the CSFs of a particular/multiple organisation. On the contrary, other modelling approached are not capable to establish the strength of causal relations among CSFs. However, 
Grey-DEMATEL a causal modelling technique can precisely determine their strength of causal relations and measure the uncleanness in data too.

\subsection{Research gaps and highlights}

Based on literature review, this work lists the following research gaps:

$>$ The business organisations are reluctant to adopt sustainable initiatives in their supply chain planning (Al Zaabi et al., 2013; Sajjad et al., 2015). The reasons for the same can be listed as: a lack of knowledge of sustainability adoption; lack of economic benefits achieved through sustainability adoption; an incomplete understanding of the various probable factors critical to adopt sustainability in supply chains (Ageron et al., 2012; Wittstruck and Teuteberg, 2012; Grimm et al., 2014). The understanding of interactive relations among the factors to adopt sustainability initiatives in a supply chains is also highly important (Wittstruck and Teuteberg, 2012; Gopal and Thakkar, 2016b).

The literature on the sustainability has grown over the past two decades or so, as mentioned in the study conducted by Fahimnia et al. (2015). However, there is a paucity of research on sustainability initiatives in developing nations, like India (Gopal and Thakkar, 2016a). Few authors also have focused on sustainable supply chains in Indian scenario and suggested that the subject of sustainability in Indian supply chains is at a very initial phase (Gupta and Palsule-Desai, 2011; Mitra and Datta, 2014; Mangla et al., 2015; Kumar and Rahman, 2016). Industries in developing countries, such as India face pressure from various perspectives to adopt sustainability initiatives in traditional supply chains (Diabat et al., 2014; Mani et al., 2016). The analysis of extant literature indicates that sustainability initiatives in supply chains in a developing country like India are not only at an early phase but also highly unorganised. Hence, it is needed to develop a framework for effective adoption of sustainability initiatives in supply chains.

Literature also suggested the need of analysing the concept of supply chain sustainability form system perspective instead of individual stakeholder viewpoint (Ogunbiyi et al., 2014). In this work, from an organisational supply chain context, the system (supply chain) is considered as combination of the people, processes and environment that work together to accomplish a desired outcome of sustainability. In today's complex environment, most supply chain activities, such as purchasing, marketing, production, are 
quite complex. The ability to visualise the functions of a supply chain, the interaction between the functions, and knowledge of the external influences on the supply chain system directly affects ability to understand the level of complexity, and cognitive ability and cause and effect relations among system elements.

In this work, sustainability oriented CSFs have been considered to assess the complexity in the process of adding sustainability in a supply chain by knowing its various related functions, interaction between these functions, and external influences (Mangla et al., 2016). This complexity is addressed by developing an initial understating on the term 'sustainability' and analysing its implications for improving ecological, economic, social gains (Sarkis, 2012) of Indian industries, so as industrial system may have variety of sustainability implications. To help managers in sustainability of supply chains, this work further uncovers the causal relations among CSFs using grey based DEMATEL approach. In line with this, present research also conducts sensitivity analysis and test the developed framework for assessing the complex causal relations among CSFs under different conditions.

\section{Critical Success Factors for Sustainability initiatives in Supply Chains}

The critical success factor theory is useful in understanding the importance of process improvement for a business organisation (Haleem et al., 2012). The concepts of key success factor theory are generally backed by the strategy research and determine the process, activities, and means to enhance the organisation's competitiveness (De Vasconcellos et al., 1989; Dinter, 2013). In this sense, theoretical developments in the subjects of sustainability initiatives implementation CSFs related to its identification, need and importance is important in incorporating sustainability initiative in supply chains. In this sense, we investigate the previous studies by searching various key words e.g. Critical Success Factors/Key Success Factors for Sustainability Initiatives in Supply Chains etc. Various search databases like Science Direct; ISI WoS; Emerald; Scopus; Taylor \& Francis; DOAJ; EBSCO, and Wiley and Inderscience were used. As a resultant, sixteen important CSFs for effective adoption of sustainability initiatives in supply chains were identified through literature and are explained in the subsequent subsections. The identified CSFs were also validated through experts' inputs (for details see Section 6.1). 


\subsection{Government legalisation}

Legislation refers to a set of laws or acts passed by regulatory bodies or government to make sure that business organisations take the responsibilities of product after the end of their useful life (Rahman and Subramanian, 2012). In recent years, a variety of laws and regulations have been passed to encourage ecological and societal sustainability e.g. the European Directives on Waste and Electronic Equipment (Bose and Pal, 2012). Government of India recently directed automotive industries to follow environment friendly Bharat Stage (BS) IV emission system (Arora, 2016).

\subsection{Top management support}

Sustainability is an imperative aspect of an organisation's mission, which results from the CEO's and top management support (Klassen, 2001). Implementation of SSCM practices is a verdict that needs to be supported by the top management of a business organisation (Ageron, et al., 2012). Top management approach and support usually determines the success possibility of adoption of SSCM initiatives at industrial standpoint (Muduli et al., 2013).

\subsection{Ecological considerations in organisations' policies and missions}

Ecological considerations include the environmental budget, environmental certification and environmental compliance; these dimensions measure the impacts of these practices on environmental protection (Baumann and Genoulaz, 2014). Organisations may facilitate green practices by establishing an environmental policy for its suppliers as a manifestation of its position regarding green purchasing, green design, green manufacturing and supplier auditing (Garetti and Taisch, 2012; Chuang et al. 2014; Gandhi et al., 2016), so as ecological practices have a positive effect on the sustainable supply chain (Ashby et al., 2012). Research and development (R\&D) plays a decisive role in the successful implementation of new ideas, technologies, and/or methods in implementing green/sustainable aspects in supply chains (Bose and Pal, 2012).

\subsection{Societal considerations}

With growing concern about ecological issues and community awareness, the public now distinguishes organisations that reveal supplementary information about their operations concerns 
in society (Zhang et al., 2017). Walker et al. (2008) recognised that pressure from a variety of sources, such as Non-Government Organisations (NGOs) and green promotion groups pressurise business organisations to critically consider their ecological and societal sustainability plans. The comprehensive social deliberations may include occupational health and safety practices, local society issues and employability practices etc. (Bai and Sarkis, 2010).

\subsection{Supply chain members' collaborations}

Environmental collaboration of an industry with its suppliers and customers is the prime requirement in implement SSCM practices (Boer et al., 2005; Vachon and Klassen, 2008; Ramanathan et al., 2014). Collaboration among supply chain members is one of the key elements in developing new technologies, processes, and products (Soosay et al., 2008; Beske et al., 2014). Business organisation may push suppliers to implement environmental and social friendly technologies and practices, which may help to reduce GHG emissions in addition to a favoured impact on the environment in the supply chain (Hassini et al., 2012).

\subsection{Technology development and process innovation}

Technology development may be utilised to solve environment and social issues, and related problems (Andiç et al., 2012). Sustainability in supply chains largely depends upon the support of partnering organisations and the use of the technology and related aspects in business (Gunasekaran and Spalanzani, 2012). Business organisations desire reducing pollution in their organisation, which essentially involves adjusting their manufacturing technology (Muduli et al., 2013). In addition to technology development, process innovation is very significant for supply chain sustainability (Ogunbiyi et al., 2014). Process innovation facilitates the adoption of innovative based practices, such as lean and green techniques, which leads to synergy to organisational efforts to accomplish economic, environmental and social goals (Miller et al., 2010).

\subsection{Communication and information technology}

Accurate information reduces uncertainty associated with the supply chain network, and collaboration through electronic media enables timely communication and information sharing among supply chain partners for sustainable business development (Prakash and Barua, 2015). 


\subsection{Green design and purchasing}

Integrating environmental concerns in the design phase of a product can reduce its negative environmental impacts, such as waste management, pollution control, life-cycle analysis and resource conservation (Zhu et al., 2007; Jabbour and Jabbour, 2009; Eltayeb et al., 2011). Green purchasing is an expensive task, but creates economic value, in terms of higher resource conservation and sustainable business development (Min and Galle, 2001; Govindan et al., 2015).

\subsection{Reverse logistics and waste minimisation}

Majority of the organisations realise that reverse logistics adoption is not only helpful in achieving sustainable business practices, but also useful in increasing revenue and corporate image (Prakash and Barua, 2015). The benefits of implementing reverse logistics operations are efficient resource utilisation, environmental protection and waste minimisation (Gunasekaran and Spalanzani, 2012).

\subsection{Ethical and safe practices}

The creation and adoption of ethical and safe practices in firms include industry image and reputation, government legislation and other stakeholders' expectations (Mzembe et al., 2016). Due to the global issues of climate change, exhaustion of resources and widespread poverty, it is important for the business corporation to develop a sense of accountability and responsibility towards their stakeholders and society at large. Businesses need to show a high level of ethics in their decision-making, thus moving beyond the core objective of profit maximisation. This is 
significant to guarantee the continued support and confidence of the stakeholders and, consequently, the sustainability of the organisation (Büyüközkan and Cifci, 2012).

\subsection{Customer involvement and encouragement}

Many organisations have been facing pressure greater than before from their major customers to perform business in a sustainable way (Dües et al., 2013). Customers may put pressure on a business organisation, which produces higher ecological and societal impacts (Deephouse and Heugens, 2009; Kumar et al., 2014; Kumar et al., 2016). Customer pressure is a key driver to encourage business organisations to develop sustainability focused practices in supply chains (Gualandris and Kalchschmidt, 2014).

\subsection{Community welfare and development}

Business growth has direct relationships with community and societal development. Transparency and equity dimensions have critical roles in the creation of a brand image of the organisation. A better quality of life for the community can aid in maintaining the cultural diversity as well as social stability (Seghezzo, 2009). This factor also differentiates one organisation from other organisations. Sustainable development encourages community development that leads to high business growth (Eltayeb et al., 2011; Khavul and Bruton, 2013).

\subsection{Economic considerations}

The implementation of sustainability focused initiatives may help in achieving financial benefits, expansion of the market by adding new customers, enhancement of sustainable capabilities and bringing competitive advantages. Therefore, many business organisations are adopting sustainable supply chain initiatives to improve their overall performance (Ageron et al., 2012; Gopalakrishnan et al., 2012).

\subsection{Competitiveness and brand image considerations}

Due to growing awareness of environmental impacts, business organisations are seeking to adopt sustainability in supply chains (Zailani et al, 2012). Sustainability issues must be considered throughout the supply chain design to confirm sustainable development in order to accomplish viable and competitive performance objectives (Gunasekaran and Spalanzani, 2012). 


\subsection{Investment recovery}

Investment recovery means recovering the organisation's investment in terms of higher inventories, scrap and excess capital equipment (Zhu et al., 2013). Investment recovery will enhance an organisation's economic performance that helps in assessing the optimal level of investment for sustainability initiatives in the supply chain (Chaabane et al., 2012).

\section{Proposed Research Framework}

Based on the literature and expert inputs, CSFs of successful implementation of sustainable initiatives in supply chains were identified. The finalised CSFs were analysed using the grey based DEMATEL approach through expert's inputs. The projected research framework is shown in Figure 1.

[Figure 1 about here]

\section{Solution Methodology}

For accomplishing the purpose of the present research, the Grey based DEMATEL approach has been suggested as a solution methodology. Majority of the Multiple-Attribute Decision Making (MADM) approaches assume that the criteria are independent of each other, which is not a realistic assumption in real world problems (Gölcük and Baykasoğlu, 2016). DEMATEL is a technique that determines the interdependence among the factors with the help of a casual diagram (Seleem et al., 2016). In practical situations, unpredictable surroundings may result in imprecise human judgments and vague information. Thus, the usual DEMATEL (Gandhi et al., 2015; Xia et al., 2015) technique is not capable of handling these uncertainties. 


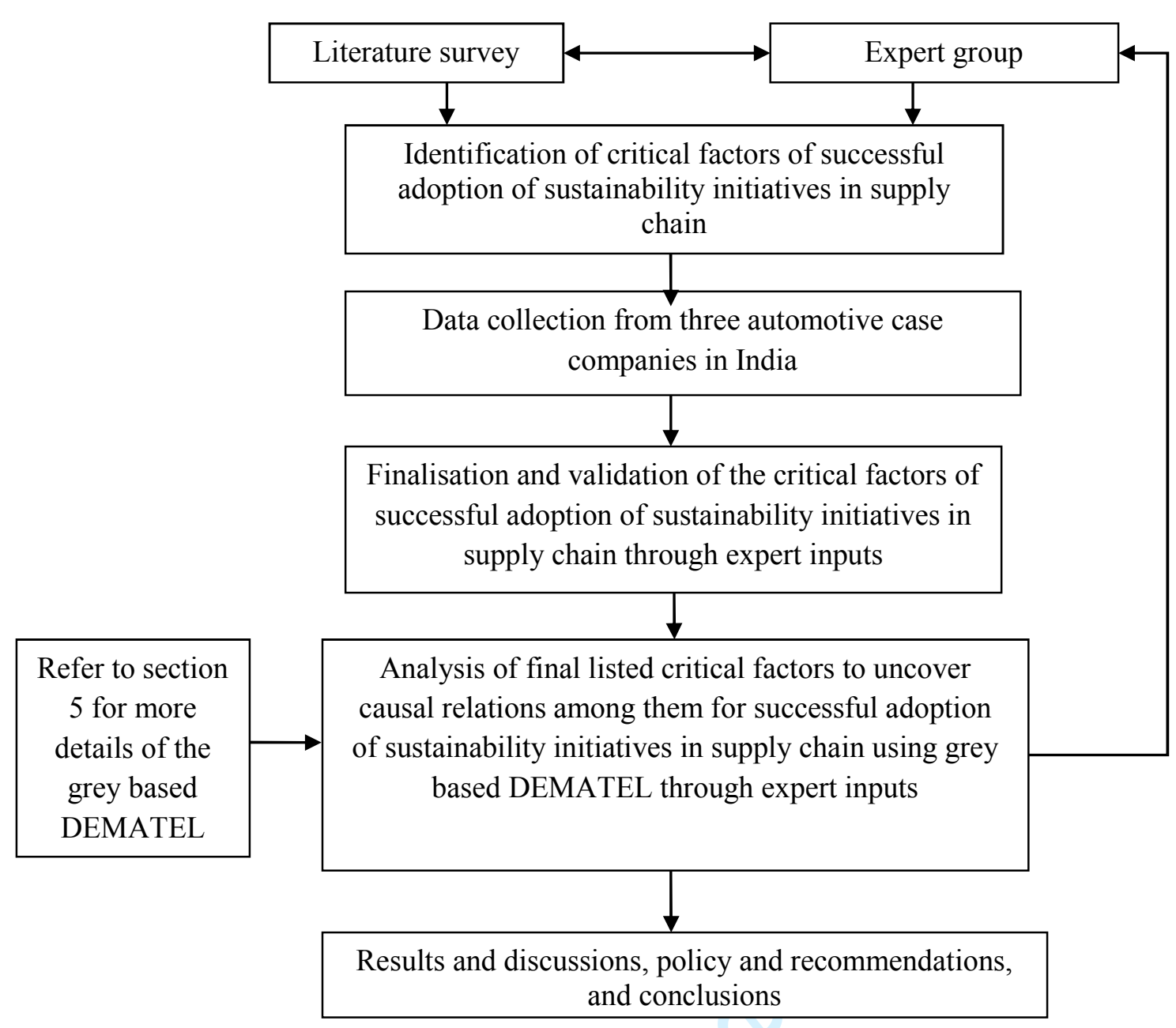

Figure 1: Proposed research framework

We may use fuzzy theory in this situation; however, fuzzy theory has some limitations in mapping a membership function (Khompatraporn and Somboonwiwat, 2017), like triangular, trapezoidal etc. Hence, this work opted to mix the grey set theory with DEMATEL. The grey system also considers the condition of fuzziness. The concept of grey set theory has been introduced by Prof. Deng (Deng, 1982). Grey theory can be readily combined with different decision making processes to advance the accuracy of the judgments (Liu et al., 2010). Grey based DEMATEL approach can uncover the causal relationships among the CSFs effective adoption of sustainability initiatives in supply chains (Bai and Sarkis. 2013). Based on the literature, researchers have used the Grey-DEMATEL methodology in different contexts (Xia et al., 2015; Su et al. 2016; Seker et al., 2017). The procedure for Grey-DEMATEL method is described as below. 
Step 1: Construct the initial relationship matrix (R). Let the number of identified CSFs for effective adoption of sustainability initiatives in supply chains be 'c' and the respondents chosen to be ' $n$ '. Each respondent is given the task of evaluating the direct influence of factor ' $x$ ' over factor ' $y$ ' on an integer scale as given in Table 2 among the 'c' factors.

Table 2: Linguistics assessment and associated Grey scales

\begin{tabular}{l|c|c}
\hline Linguistics assessment & Assigned Grey numbers & Crisp values \\
\hline No influence (N) & $(0,0.1)$ & 0 \\
\hline Very low influence (VL) & $(0.1,0.3)$ & 1 \\
\hline Low influence (L) & $(0.2,0.5)$ & 2 \\
\hline Medium influence (M) & $(0.4,0.7)$ & 4 \\
\hline High influence (H) & $(0.6,0.9)$ & 5 \\
\hline Very high influence (VH) & $(0.9,1.0)$ & \\
\hline
\end{tabular}

Step 2: Calculate the corresponding Grey matrices $\left(\otimes A_{x y}^{l}\right)$. The initial relationship matrices are transformed into corresponding grey matrices. For this, the integer scale ratings are converted into associated Grey numbers based on an upper and lower range of values, as given in Table 2 (Deng, 1982; Rajesh et al., 2015), i.e.

$\otimes A_{x y}^{l}=\left(\underline{\otimes} A_{x y}^{l}, \bar{\otimes} A_{x y}^{l}\right)$

Where $1 \leq 1 \leq \mathrm{n} ; 1 \leq \mathrm{x} \leq \mathrm{c} ; 1 \leq \mathrm{y} \leq \mathrm{c}$.

Step 3: Determine the average Grey matrix $\left(\otimes \check{A}_{x y}\right)$, that is prepared by taking the average of initial Grey matrices using equation (5.2).

$\otimes \check{A}_{x y}=\left(\sum_{l} \frac{\otimes}{n} A_{x y}^{l}, \sum_{l} \frac{\bar{\otimes} A_{x y}^{l}}{n}\right)$

Step 4: Transform the average Grey matrix into crisp relationship matrix (B). The Grey numbers are converted to crisp numbers by the modified-CFCS (Converting Fuzzy data into Crisp Scores) (Xia et al., 2015). For other details about formation of crisp relationship matrix (B) refer to Appendix-A.

Step 5: Set up the normalised direct-relation matrix (N). Based on equations (5.3) and (5.4), the normalised direct relation matrix is constructed.

$$
\begin{aligned}
& L=\frac{1}{\max _{1 \leq x \leq c}^{c} \sum_{y}^{c} a_{x y}} \\
& N=L^{*} R
\end{aligned}
$$

Where, $\mathrm{L}$ is the normalisation factor and $\mathrm{R}$ is initial relationship matrix. 
Step 6: Determine total relation matrix (T) by using equation (5.5).

$$
T=N(I-N)^{-1}
$$

Where, $\mathrm{I}$ is the identity matrix.

Step 7: Obtain causal parameters. ' $\mathrm{D}$ ' denotes the summation of rows and ' $R$ ' denotes the summation of columns. This is calculated through equations (5.6) and (5.7):

$$
\begin{aligned}
& R=\left[\sum_{y=1}^{c} a_{x y}\right]_{c \times 1} \\
& D=\left[\sum_{y=1}^{c} a_{x y}\right]_{1 \times c}
\end{aligned}
$$

Step 8: Set up the causal diagram. A causal and effect diagram is constructed through dataset consisting of $(\mathrm{R}+\mathrm{D}, \mathrm{R}-\mathrm{D})$. The score $(\mathrm{R}+\mathrm{D})$ denotes 'Prominence' and implies the total effects given and received by factor ' $\mathrm{x}$ ', whereas the score (R-D) denotes 'Relation' of one factor with other.

\section{An Application of Proposed Framework}

A multiple case study approach of three automotive component manufacturing companies from India is conducted in this work. Case study approach is useful in demonstrating real world phenomena (Subramanian et al., 2014). In this work, we select three case companies to generalise our study outcomes in achieving sustainability of supply chains (Eisenhardt, 1989; Yin, 2013). In general, our findings are effective to the limited context of the preferred companies but provide basis for future studies that may be generalised to larger populations.

In this work, three automotive companies produce a wide variety of products, including highly precise and fully machined aluminum and ferrous components for automotive Original Equipment Manufacturers (OEMs). The case companies uphold high standards of business ethics and social responsibility, continually innovating the processes and products in partnership with suppliers to attain improved performance. Top management of the case companies is committed to sustainable business development and they are involved in a project "Sustainable Supply Chain Management Implementation". Management also intends to identify and analyse CSFs to uncover the causal relations among the CSFs for successful sustainability initiatives in the supply chain.

Brief explanation of case companies considered in this research is provided in Table 3. 
Table 3: Brief description of case companies

\begin{tabular}{l|l|l|l}
\hline $\begin{array}{l}\text { Business } \\
\text { Characteristics }\end{array}$ & Company 1 & Company 2 & Company 3 \\
\hline Turnover (in INR) & $150-160$ Million & $140-150$ Million & $120-130$ Million \\
\hline Employees & More than 2000 & More than 2000 & $1500-2000$ \\
\hline $\begin{array}{l}\text { Year of } \\
\text { establishment }\end{array}$ & 1983 & 1984 & 1987 \\
\hline Certifications & OHSAS 18001 and ISO 14001 & $\begin{array}{l}\text { ISO 14001, ISO TS } \\
16949, \text { OHSAS 18001 }\end{array}$ & $\begin{array}{l}\text { ISO 9001, ISO 14001, TS } \\
16949 \text { and OHSAS 18001 }\end{array}$ \\
\hline $\begin{array}{l}\text { Products } \\
\text { manufactured type/ } \\
\text { Specialization }\end{array}$ & $\begin{array}{l}\text { Various automotive (2,3,4 and } \\
\text { commercial wheelers) }\end{array}$ & 2 wheeler components & $\begin{array}{l}2 \text { and 4 wheelers automotive } \\
\text { components }\end{array}$ \\
\hline $\begin{array}{l}\text { Type of business } \\
\text { componts }\end{array}$ & \begin{tabular}{l} 
Manufacturer, supplier \\
\hline
\end{tabular} & Manufacturer, supplier & Manufacturer, supplier \\
\hline
\end{tabular}

A group comprising 17 experts (details are provided in next section) was formed. In this work, the system (supply chain of automotive companies under study) is considered as combination of the people, processes and environment that work together to accomplish a desired outcome of sustainability. The application of the proposed framework is elaborated in the subsequent subsections.

\subsection{Finalisation and validation of the CSFS}

Initially, 16 sustainability focused CSFs in supply chains were listed from the literature. To validate the identified factors, a feedback form was prepared as shown in Appendix-B. The feedback allows ranking of the expert's rating in terms of importance of each factor on a scale of $1-7$ (where, 1-least relevant and 7-most relevant). The expert panel comprised 17 people: 9 business professionals dealing with implementation of sustainability issues in automotive supply chain, 4 sustainability management consultants, 4 representatives from national and regional public institutions dealing with environmental issues, and 3 faculty members actively conducting research on sustainability management issues. The experts selected were knowledgeable and skilled based professionals, with more than ten years of working experience in the domain of management of sustainability issues. The experts' responses were gathered to finalise the sustainability initiatives related factors in the automotive industry in the Indian context. Based on expert's agreement, we deleted the factors with a rating of 1 or 2; thus, one factor was eliminated, i.e. 'Investment Recovery'. This means, currently, 'Investment Recovery' is relatively less 
significant as compared to other factors. The experts were agreed on the point that investment recovery certainly enriches the business sustainability initiatives by holding the sustainability concept of the 3 R's: (reduce, reuse, and recycle). However, investment recovery is less important in developing nations, such as India so as to very initial level of sustainable initiatives in supply chain context (Zhu et al., 2013). The case companies also have limited resources capabilities in infrastructure and waste management policies. Therefore, presently, investment recovery concepts are very weak in improving the sustainability of Indian case automotive company value chains. In view of this, we left out this factor in the current research with an aim to evaluate the effect of investment recovery in business sustainability of Indian automotive companies and related industry in future studies.

We also asked experts to add/include any other CSF, which they thought is significant in sustainability of supply chains, however, some CSFs were reworded to suite with Indian supply chain context. All in all, they seem to be satisfied with the list and were not agreed for including any other. Hence, a total of $15 \mathrm{CSF}$ relevant to the implementation of sustainability initiatives in the automotive industry supply chain were selected.

\subsection{Uncovering causal relations of CSFs}

The grey based DEMATEL approach was utilised to uncover causal relations among the CSFs. Thus, the expert group was asked to rate the CSFs using the linguistic scale shown in Table 2. Based on this, initial direct matrices were formed. Next, the initial relationship matrices were transformed into corresponding grey matrices by assigning Grey values of the linguistic scales using equation 5.1. The Grey relationship matrix for critical factors towards effective adoption of sustainability initiatives in the automotive industry supply chain by Expert 1 is given in Table 4.

[Table 4 about here]

Next, the average Grey relation matrix $\left[\otimes \check{A}_{x y}\right]$ was computed. The average Grey relation matrix is shown in Table 5.

[Table 5 about here]

The normalised crisp relation matrix (B) was constructed from the average Grey relation matrix using the modified-CFCS method and is shown in Table 6. 


\section{[Table 6 about here]}

The final crisp relation matrix (B) was constructed from the average Grey relation matrix and is shown in Table 7.

\section{[Table 7 about here]}

Next, the normalised direct relation matrix $(\mathrm{N})$ was constructed and is given in Table 8 .

$$
\text { [Table } 8 \text { about here] }
$$

The total relation matrix $\mathrm{T}$ is obtained from normalised direct relation matrix and is shown in Table 9.

[Table 9 about here]

Let $\mathrm{R}$ and $\mathrm{D}$ be defined as $\left[\mathrm{r}_{\mathrm{x}}\right]_{\mathrm{n} \times 1}$ and $\left[\mathrm{c}_{\mathrm{y}}\right]_{1 \times \mathrm{n}}$ vectors representing the sum of the row elements and the sum of the column elements for the total relation matrix T, respectively. The 'Prominence' $(\mathrm{R}+\mathrm{D})$ and 'Relation' (R-D) were computed by adding and subtracting the values of $\mathrm{R}$ and $\mathrm{D}$; other details are shown in Table 10.

\section{[Table 10 about here]}

Finally, a causal effect diagram of factors for successful adoption of sustainability initiatives in the supply chain is plotted by taking the dataset consisting of $(\mathrm{R}+\mathrm{D}, \mathrm{R}-\mathrm{D}$. To show the net effect and correlation among all the CSFs and in the sets, a causal and effect diagram is developed (Please see Figure 2).

\section{[Figure 2 about here]}

From Figure 2, eleven CSFs have been categorised into the cause group and four CSFs into the effect group. The relationships among CSFs are shown through arrows in digraphs (Figure 2).

Threshold value $(\alpha)$ has been fixed to sort out number of relationships which have higher value than $\alpha$. The threshold value is calculated by adding one standard deviation to the mean. In this case, $\alpha$ is 0.1201 i.e. $(0.0805+0.0396)$. All the relationships among CSFs meeting or exceeding the threshold value are plotted in Figure 2. 
Table 4: Grey relationship matrix for CSFs for effective adoption of sustainability initiatives in the automotive industry supply chain by Expert 1

\begin{tabular}{|c|c|c|c|c|c|c|c|c|c|c|c|c|c|c|c|}
\hline CSFs & CSF1 & CSF2 & CSF3 & CSF4 & CSF5 & SF6 & CSF7 & CSF8 & SSF9 & CSF10 & CSF11 & CSF12 & CSF13 & CSF14 & CSF15 \\
\hline CSF1 & $(0,0.1)$ & $0.1,0.3)$ & $(0.2,0.5)$ & $(0.1,0.3)$ & $(0.2,0.5)$ & $(0.2,0.5)$ & $(0.4,0.7)$ & $(0.2,0.5)$ & $(0.2,0.5)$ & $(0.2,0.5)$ & $(0.2,0.5)$ & $(0.4,0.7)$ & $(0.4,0.7)$ & $(0.4,0.7)$ & $(0.2,0.5)$ \\
\hline CSF2 & $.1,0.3)$ & $(0,0.1)$ & $.2,0.5)$ & $.2,0.5)$ & $(0.4,0.7)$ & $(0.2,0.5)$ & $(0.2,0.5)$ & $(0.2,0.5)$ & $(0.1,0.3)$ & $(0.2,0.5)$ & $(0.4,0.7)$ & $(0.4,0.7)$ & $(0.4,0.7)$ & $(0.2,0.5)$ & $0.4,0.7)$ \\
\hline CSF3 & $.1,0.3)$ & $1,0.3)$ & $(0,0.1)$ & $0.2,0.5)$ & $(0.2,0.5)$ & $(0.2,0.5)$ & $(0.2,0.5)$ & $(0.2,0.5)$ & $(0.1,0.3)$ & $(0.1,0.3)$ & $(0.4,0.7)$ & $(0.1,0.3)$ & $(0.4,0.7)$ & $(0.2,0.5)$ & $(0.4,0.7)$ \\
\hline CSF4 & $(0.1,0.3)$ & $(0.1,0.3)$ & $(0.2,0.5)$ & $(0,0.1)$ & $(0.2,0.5)$ & $(0.2,0.5)$ & $(0.4,0.7)$ & $(0.1,0.3)$ & $(0.4,0.7)$ & $(0.1,0.3)$ & $(0.4,0.7)$ & $(0.1,0.3)$ & $(0.2,0.5)$ & $(0.4,0.7)$ & $(0.2,0.5)$ \\
\hline CSF5 & $(0.1,0.3)$ & $(0.2,0.5)$ & $(0.2,0.5)$ & $(0.1,0.3)$ & $(0,0.1)$ & $(0.2,0.5)$ & $(0.2,0.5)$ & $(0.1,0.3)$ & $(0.2,0.5)$ & $(0.1,0.3)$ & $(0.4,0.7)$ & $(0.1,0.3)$ & $(0.4,0.7)$ & $(0.2,0.5)$ & $(0.4,0.7)$ \\
\hline CSF6 & $(0.2,0.5)$ & $(0.1,0.3)$ & $(0.1,0.3)$ & $(0.2,0.5)$ & $(0.2,0.5)$ & $(0,0.1)$ & $(0.2,0.5)$ & $(0.2,0.5)$ & $(0.2,0.5)$ & $(0.2,0.5)$ & $(0.4,0.7)$ & $(0.2,0.5)$ & $(0.4,0.7)$ & $(0.4,0.7)$ & $(0.4,0.7)$ \\
\hline CSF7 & $(0.2,0.5)$ & $(0.1,0.3)$ & $(0.2,0.5)$ & $(0.2,0.5)$ & $(0.2,0.5)$ & $(0.1,0.3)$ & $(0,0.1)$ & $(0.1,0.3)$ & $(0.2,0.5)$ & $(0.1,0.3)$ & $(0.6,0.9)$ & $(0.1,0.3)$ & $(0.4,0.7)$ & $(0.4,0.7)$ & $(0.4,0.7)$ \\
\hline CSF8 & $(0.1,0.3)$ & $(0.1,0.3)$ & $(0.1,0.3)$ & $(0.1,0.3)$ & $(0.2,0.5)$ & $(0.2,0.5)$ & $(0.4,0.7)$ & $(0,0.1)$ & $(0.1,0.3)$ & $(0.4,0.7)$ & $(0.4,0.7)$ & $(0.2,0.5)$ & $(0.4,0.7)$ & $(0.4,0.7)$ & $(0.4,0.7)$ \\
\hline CSF9 & $(0.1,0.3)$ & $(0.1,0.3)$ & $(0.2,0.5)$ & $(0.1,0.3)$ & $(0.1,0.3)$ & $(0.2,0.5)$ & $(0.1,0.3)$ & $(0.1,0.3)$ & $(0,0.1)$ & $(0.4,0.7)$ & $(0.4,0.7)$ & $(0.1,0.3)$ & $(0.4,0.7)$ & $(0.4,0.7)$ & $(0.4,0.7)$ \\
\hline CSF10 & $(0.1,0.3)$ & $(0.1,0.3)$ & $(0.2,0.5)$ & $(0.2,0.5)$ & $(0.2,0.5)$ & $(0.1,0.3)$ & $(0.2,0.5)$ & $(0.2,0.5)$ & $(0.4,0.7)$ & $(0,0.1)$ & $(0.4,0.7)$ & $(0.1,0.3)$ & $(0.4,0.7)$ & $(0.2,0.5)$ & $(0.6,0.9)$ \\
\hline CSF11 & $(0,0.1)$ & $(0.1,0.3)$ & $(0.1,0.3)$ & $(0.2,0.5)$ & $(0.2,0.5)$ & $(0.1,0.3)$ & $(0.2,0.5)$ & $(0.2,0.5)$ & $(0.1,0.3)$ & $(0.2,0.5)$ & $(0,0.1)$ & $(0.2,0.5)$ & $(0.4,0.7)$ & $(0.4,0.7)$ & $(0.4,0.7)$ \\
\hline CSF12 & $(0.2,0.5)$ & $(0.2,0.5)$ & $(0.1,0.3)$ & $(0.1,0.3)$ & $(0,0.1)$ & $(0.2,0.5)$ & $(0,0.1)$ & $(0.2,0.5)$ & $(0.1,0.3)$ & $(0.2,0.5)$ & $(0.6,0.9)$ & $(0,0.1)$ & $(0.6,0.9)$ & $(0.6,0.9)$ & $(0.4,0.7)$ \\
\hline CSF13 & $(0,0.1)$ & $(0.1,0.3)$ & $(0.1,0.3)$ & $(0.1,0.3)$ & $(0.2,0.5)$ & $(0.1,0.3)$ & $(0.2,0.5)$ & $(0.1,0.3)$ & $(0.2,0.5)$ & $(0.1,0.3)$ & $(0.2,0.5)$ & $(0.1,0.3)$ & $(0,0.1)$ & $(0.6,0.9)$ & $(0.6,0.9)$ \\
\hline CSF14 & $(0.1,0.3)$ & $(0.2,0.5)$ & $(0.1,0.3)$ & $(0.1,0.3)$ & $(0.1,0.3)$ & $(0.1,0.3)$ & $(0.1,0.3)$ & $(0.2,0.5)$ & $(0.1,0.3)$ & $(0.1,0.3)$ & $(0.2,0.5)$ & $(0.1,0.3)$ & $(0.4,0.7)$ & $(0,0.1)$ & $(0.4,0.7)$ \\
\hline CSF15 & $(0.1,0.3)$ & $(0.1,0.3)$ & $(0.1,0.3)$ & $(0.2,0.5)$ & $(0.1,0.3)$ & $(0.1,0.3)$ & $(0.2,0.5)$ & $(0,0.1)$ & $(0.2,0.5)$ & $(0.1,0.3)$ & $(0.2,0.5)$ & $(0.1,0.3)$ & $(0.4,0.7)$ & $(0.6,0.9)$ & $(0,0.1)$ \\
\hline
\end{tabular}

The level of influence of factor $\mathrm{x}$ the over the factor $\mathrm{y}$ is represented as Grey value $\left(\underline{\otimes} A_{x y}^{l}, \bar{\otimes} A_{x y}^{l}\right)$

Table 5: Average Grey relationship matrix for CSFs for effective adoption of sustainability initiatives in the automotive industry supply chain

\begin{tabular}{|c|c|c|c|c|c|c|c|c|c|c|c|c|c|c|c|}
\hline CSFs & CSF1 & $\begin{array}{l}\text { CSF2 } \\
\text {. }\end{array}$ & $\begin{array}{l}\text { CSF3 } \\
\end{array}$ & CSF4 & $\begin{array}{l}\text { CSF5 } \\
\end{array}$ & CSF6 & CSF7 & CSF8 & CSF9 & CSF10 & CSF11 & CSF12 & CSF13 & CSF14 & CSF15 \\
\hline CSF1 & $(0,0.1)$ & $(0.188,0.475)$ & $(0.35,0.65)$ & $(0.188,0.475)$ & $(0.2,0.5)$ & $(0.2,0.5)$ & $(0.375,0.675)$ & $(0.2,0.5)$ & $(0.2,0.5)$ & $(0.2,0.5)$ & $(0.35,0.65)$ & $(0.35,0.65)$ & $(0.275,0.575)$ & $(0.275,0.575)$ & $(0.225,0.525)$ \\
\hline $\begin{array}{l}\text { CSF2 } \\
\end{array}$ & $(0.188,0.475)$ & $(0,0.1)$ & $(0.35,0.65)$ & $(0.2,0.5)$ & $(0.375,0.675)$ & $(0.2,0.5)$ & $(0.2,0.5)$ & $(0.2,0.5)$ & $(0.1,0.3)$ & $(0.2,0.5)$ & $(0.3,0.6)$ & $(0.35,0.65)$ & $(0.375,0.675)$ & $(0.2,0.5)$ & $(0.375,0.675)$ \\
\hline CSF3 & $(0.188,0.475)$ & $(0.188,0.475)$ & $(0,0.1)$ & $(0.2,0.5)$ & $(0.2,0.5)$ & $(0.2,0.5)$ & $(0.2,0.5)$ & $(0.2,0.5)$ & $(0.1,0.3)$ & $(0.1,0.3)$ & $(0.4,0.7)$ & $(0.2,0.5)$ & $(0.4,0.7)$ & $(0.2,0.5)$ & $(0.4,0.7)$ \\
\hline CSF4 & $(0.1,0.3)$ & $(0.1,0.3)$ & $(0.2,0.5)$ & $(0,0.1)$ & $(0.2,0.5)$ & $(0.2,0.5)$ & $(0.4,0.7)$ & $(0.1,0.3)$ & $(0.4,0.7)$ & $(0.1,0.3)$ & $(0.4,0.7)$ & $(0.1,0.3)$ & $(0.2,0.5)$ & $(0.4,0.7)$ & $(0.2,0.5)$ \\
\hline CSF5 & $(0.1,0.3)$ & $(0.2,0.5)$ & $(0.2,0.5)$ & $(0.1,0.3)$ & $(0,0.1)$ & $(0.2,0.5)$ & $(0.2,0.5)$ & $(0.1,0.3)$ & $(0.2,0.5)$ & $(0.1,0.3)$ & $(0.4,0.7)$ & $(0.1,0.3)$ & $(0.4,0.7)$ & $(0.2,0.5)$ & $(0.4,0.7)$ \\
\hline CSF6 & $(0.2,0.5)$ & $(0.1,0.3)$ & $(0.1,0.3)$ & $(0.2,0.5)$ & $(0.2,0.5)$ & $(0,0.1)$ & $(0.2,0.5)$ & $(0.2,0.5)$ & $(0.2,0.5)$ & $(0.2,0.5)$ & $(0.525,0.825)$ & $(0.2,0.5)$ & $(0.575,0.875)$ & $(0.4,0.7)$ & $(0.575,0.875)$ \\
\hline CSF7 & $(0.2,0.5)$ & $(0.1,0.3)$ & $(0.2,0.5)$ & $(0.2,0.5)$ & $(0.2,0.5)$ & $(0.1,0.3)$ & $(0,0.1)$ & $(0.2,0.5)$ & $(0.2,0.5)$ & $(0.1,0.3)$ & $(0.55,0.85)$ & $(0.1,0.3)$ & $(0.4,0.7)$ & $(0.4,0.7)$ & $(0.4,0.7)$ \\
\hline CSF8 & $(0.1,0.3)$ & $(0.1,0.3)$ & $(0.1,0.3)$ & $(0.1,0.3)$ & $(0.2,0.5)$ & $(0.2,0.5)$ & $(0.4,0.7)$ & $(0,0.1)$ & $(0.125,0.35)$ & $(0.4,0.7)$ & $(0.575,0.875)$ & $(0.2,0.5)$ & $(0.575,0.875)$ & $(0.4,0.7)$ & $(0.4,0.7)$ \\
\hline CSF9 & $(0.125,0.35)$ & $(0.125,0.35)$ & $(0.2,0.5)$ & $(0.1,0.3)$ & $(0.1,0.3)$ & $(0.2,0.5)$ & $(0.1,0.3)$ & $(0.125,0.35)$ & $(0,0.1)$ & $(0.525,0.825)$ & $(0.4,0.7)$ & $(0.1,0.3)$ & $(0.575,0.875)$ & $(0.575,0.875)$ & $(0.4,0.7)$ \\
\hline CSF10 & $(0.113,0.325)$ & $(0.138,0.375)$ & $(0.2,0.5)$ & $(0.2,0.5)$ & $(0.2,0.5)$ & $(0.1,0.3)$ & $(0.2,0.5)$ & $(0.2,0.5)$ & $(0.5,0.8)$ & $(0,0.1)$ & $(0.4,0.7)$ & $(0.1,0.3)$ & $(0.4,0.7)$ & $(0.2,0.5)$ & $(0.6,0.9)$ \\
\hline CSF11 & $(0,0.1)$ & $(0.1,0.3)$ & $(0.1,0.3)$ & $(0.2,0.5)$ & $(0.2,0.5)$ & $(0.1,0.3)$ & $(0.2,0.5)$ & $(0.2,0.5)$ & $(0.1,0.3)$ & $(0.2,0.5)$ & $\begin{array}{l}(0,0.1) \\
\end{array}$ & $(0.2,0.5)$ & $\begin{array}{l}(0.5,0.85) \\
\end{array}$ & $(0.4,0.7)$ & $(0.4,0.7)$ \\
\hline CSF12 & $(0.2,0.5)$ & $(0.2,0.5)$ & $(0.1,0.3)$ & $(0.1,0.3)$ & $(0,0.1)$ & $(0.2,0.5)$ & $(0.1,0.3)$ & $(0.2,0.5)$ & $(0.1,0.3)$ & $(0.2,0.5)$ & $(0.575,0.875)$ & $(0,0.1)$ & $(0.6,0.9)$ & $(0.575,0.875)$ & $(0.5,0.8)$ \\
\hline CSF13 & $(0,0.1)$ & $(0.1,0.3)$ & $(0.1,0.3)$ & $(0.1,0.3)$ & $(0.2,0.5)$ & $\begin{array}{l}(0.1,0.3) \\
\end{array}$ & $(0.2,0.5)$ & $(0.1,0.3)$ & $(0.2,0.5)$ & $(0.1,0.3)$ & $(0.25,0.55)$ & $(0.1,0.3)$ & $(0,0.1)$ & $(0.45,0.75)$ & $\begin{array}{l}(0.375,675) \\
\end{array}$ \\
\hline CSF14 & $(0.1,0.3)$ & $(0.188,0.45)$ & $(0.1,0.3)$ & $(0.1,0.3)$ & $(0.1,0.3)$ & $(0.1,0.3)$ & $(0.1,0.3)$ & $(0.2,0.5)$ & $(0.1,0.3)$ & $(0.1,0.3)$ & $(0.25,0.55)$ & $(0.1,0.3)$ & $(0.4,0.7)$ & $(0,0.1)$ & $(0.35,0.65)$ \\
\hline$\overline{C S F 15}$ & $(0.088,0.25)$ & $(0.1,0.3)$ & $(0.1,0.3)$ & $(0.2,0.5)$ & $(0.1,0.3)$ & $(0.1,0.3)$ & $(0.2,0.5)$ & $(0,0.1)$ & $(0.2,0.5)$ & $(0.1,0.3)$ & $(0.275,0.575)$ & $(0.1,0.3)$ & $(0.325,0.625)$ & $(0.4,0.7)$ & $(0,0.1)$ \\
\hline
\end{tabular}

The level of influence of driver $\mathrm{x}$ the over the driver $\mathrm{y}$ is represented as Grey value $\left(\underline{\otimes} A_{x y}^{l}, \bar{\otimes} A_{x y}^{l}\right)$

\section{0}

URL: http://mc.manuscriptcentral.com/tppc E-mail: ppc@plymouth.ac.uk 
Table 6: Normalised crisp relationship matrix for CSFs for effective adoption of sustainability initiatives in the automotive industry supply chain

\begin{tabular}{|c|c|c|c|c|c|c|c|c|c|c|c|c|c|c|c|}
\hline CSFs & CSF1 & CSF2 & CSF3 & CSF4 & CSF5 & CSF6 & CSF7 & CSF8 & CSF9 & CSF10 & CSF11 & CSF12 & CSF13 & CSF14 & CSF15 \\
\hline CSF1 & 0.0000 & 0.5795 & 0.7376 & 0.5795 & 0.4317 & 0.6286 & 0.7183 & 0.6286 & 0.3500 & 0.3370 & 0.5169 & 0.7376 & 0.4015 & 0.4153 & 0.3359 \\
\hline CSF2 & 0.5795 & 0.0000 & 0.7376 & 0.6286 & 0.7503 & 0.6286 & 0.4127 & 0.6286 & 0.1528 & 0.3370 & 0.4492 & 0.7376 & 0.5328 & 0.3136 & 0.5328 \\
\hline CSF3 & 0.5795 & 0.5795 & 0.0000 & 0.6286 & 0.4317 & 0.6286 & 0.4127 & 0.6286 & 0.1528 & 0.1474 & 0.5847 & 0.4525 & 0.5657 & 0.3136 & 0.5657 \\
\hline CSF4 & 0.2667 & 0.2667 & 0.4525 & 0.0000 & 0.4317 & 0.6286 & 0.7619 & 0.2667 & 0.6500 & 0.1474 & 0.5847 & 0.1949 & 0.3030 & 0.5847 & 0.3030 \\
\hline CSF5 & 0.2667 & 0.6286 & 0.4525 & 0.2667 & 0.0000 & 0.6286 & 0.4127 & 0.2667 & 0.3500 & 0.1474 & 0.5847 & 0.1949 & 0.5657 & 0.3136 & 0.5657 \\
\hline CSF6 & 0.6286 & 0.2667 & 0.1949 & 0.6286 & 0.4317 & 0.0000 & 0.4127 & 0.6286 & 0.3500 & 0.3370 & 0.7542 & 0.4525 & 0.7955 & 0.5847 & 0.7955 \\
\hline CSF7 & 0.6286 & 0.2667 & 0.4525 & 0.6286 & 0.4317 & 0.2667 & 0.0000 & 0.2667 & 0.3500 & 0.1474 & 0.7880 & 0.1949 & 0.5657 & 0.5847 & 0.5657 \\
\hline CSF8 & 0.2667 & 0.2667 & 0.1949 & 0.2667 & 0.4317 & 0.6286 & 0.7619 & 0.0000 & 0.1985 & 0.6268 & 0.8219 & 0.4525 & 0.7955 & 0.5847 & 0.5657 \\
\hline CSF9 & 0.3500 & 0.3500 & 0.4525 & 0.2667 & 0.1864 & 0.6286 & 0.1786 & 0.3500 & 0.0000 & 0.8078 & 0.5847 & 0.1949 & 0.7955 & 0.8219 & 0.5657 \\
\hline CSF10 & 0.3077 & 0.3936 & 0.4525 & 0.6286 & 0.4317 & 0.2667 & 0.4127 & 0.6286 & 0.8000 & 0.0000 & 0.5847 & 0.1949 & 0.5657 & 0.3136 & 0.8283 \\
\hline CSF11 & 0.0000 & 0.2667 & 0.1949 & 0.6286 & 0.4317 & 0.2667 & 0.4127 & 0.6286 & 0.1528 & 0.3370 & 0.0000 & 0.4525 & 0.7367 & 0.5847 & 0.5657 \\
\hline CSF12 & 0.6286 & 0.6286 & 0.1949 & 0.2667 & 0.0000 & 0.6286 & 0.0000 & 0.6286 & 0.1528 & 0.3370 & 0.8219 & 0.0000 & 0.8283 & 0.8219 & 0.6970 \\
\hline CSF13 & 0.0000 & 0.2667 & 0.1949 & 0.2667 & 0.4317 & 0.2667 & 0.4127 & 0.2667 & 0.3500 & 0.1474 & 0.3814 & 0.1949 & 0.0000 & 0.6525 & 0.5328 \\
\hline CSF14 & 0.2667 & 0.5467 & 0.1949 & 0.2667 & 0.1864 & 0.2667 & 0.1786 & 0.6286 & 0.1528 & 0.1474 & 0.3814 & 0.1949 & 0.5657 & 0.0000 & 0.5000 \\
\hline CSF15 & 0.2083 & 0.2667 & 0.1949 & 0.6286 & 0.1864 & 0.2667 & 0.4127 & 0.0000 & 0.3500 & 0.1474 & 0.4153 & 0.1949 & 0.4672 & 0.5847 & 0.0000 \\
\hline
\end{tabular}

Table 7: Final crisp relationship matrix for CSFs for effective adoption of sustainability initiatives in the automotive industry supply chain

\begin{tabular}{|c|c|c|c|c|c|c|c|c|c|c|c|c|c|c|c|}
\hline CSFs & CSF1 & CSF2 & CSF3 & CSF4 & CSF5 & CSF6 & CSF7 & CSF8 & CSF9 & CSF10 & CSF11 & CSF12 & CSF13 & CSF14 & CSF15 \\
\hline CSF1 & 0.0000 & 0.2898 & 0.4794 & 0.2898 & 0.2914 & 0.3143 & 0.5028 & 0.3143 & 0.2800 & 0.2780 & 0.4523 & 0.4794 & 0.3614 & 0.3634 & 0.3023 \\
\hline CSF2 & 0.2898 & 0.0000 & 0.4794 & 0.3143 & 0.5064 & 0.3143 & 0.2889 & 0.3143 & 0.1222 & 0.2780 & 0.3930 & 0.4794 & 0.4795 & 0.2744 & 0.4795 \\
\hline CSF3 & 0.2898 & 0.2898 & 0.0000 & 0.3143 & 0.2914 & 0.3143 & 0.2889 & 0.3143 & 0.1222 & 0.1216 & 0.5116 & 0.2941 & 0.5091 & 0.2744 & 0.5091 \\
\hline CSF4 & 0.1333 & 0.1333 & 0.2941 & 0.0000 & 0.2914 & 0.3143 & 0.5333 & 0.1333 & 0.5200 & 0.1216 & 0.5116 & 0.1267 & 0.2727 & 0.5116 & 0.2727 \\
\hline CSF5 & 0.1333 & 0.3143 & 0.2941 & 0.1333 & 0.0000 & 0.3143 & 0.2889 & 0.1333 & 0.2800 & 0.1216 & 0.5116 & 0.1267 & 0.5091 & 0.2744 & 0.5091 \\
\hline CSF6 & 0.3143 & 0.1333 & 0.1267 & 0.3143 & 0.2914 & 0.0000 & 0.2889 & 0.3143 & 0.2800 & 0.2780 & 0.6599 & 0.2941 & 0.7159 & 0.5116 & 0.7159 \\
\hline CSF7 & 0.3143 & 0.1333 & 0.2941 & 0.3143 & 0.2914 & 0.1333 & 0.0000 & 0.1333 & 0.2800 & 0.1216 & 0.6895 & 0.1267 & 0.5091 & 0.5116 & 0.5091 \\
\hline CSF8 & 0.1333 & 0.1333 & 0.1267 & 0.1333 & 0.2914 & 0.3143 & 0.5333 & 0.0000 & 0.1588 & 0.5171 & 0.7192 & 0.2941 & 0.7159 & 0.5116 & 0.5091 \\
\hline CSF9 & 0.1750 & 0.1750 & 0.2941 & 0.1333 & 0.1258 & 0.3143 & 0.1250 & 0.1750 & 0.0000 & 0.6665 & 0.5116 & 0.1267 & 0.7159 & 0.7192 & 0.5091 \\
\hline CSF10 & 0.1538 & 0.1968 & 0.2941 & 0.3143 & 0.2914 & 0.1333 & 0.2889 & 0.3143 & 0.6400 & 0.0000 & 0.5116 & 0.1267 & 0.5091 & 0.2744 & 0.7455 \\
\hline CSF11 & 0.0000 & 0.1333 & 0.1267 & 0.3143 & 0.2914 & 0.1333 & 0.2889 & 0.3143 & 0.1222 & 0.2780 & 0.0000 & 0.2941 & 0.6630 & 0.5116 & 0.5091 \\
\hline CSF12 & 0.3143 & 0.3143 & 0.1267 & 0.1333 & 0.0000 & 0.3143 & 0.0000 & 0.3143 & 0.1222 & 0.2780 & 0.7192 & 0.0000 & 0.7455 & 0.7192 & 0.6273 \\
\hline CSF13 & 0.0000 & 0.1333 & 0.1267 & 0.1333 & 0.2914 & 0.1333 & 0.2889 & 0.1333 & 0.2800 & 0.1216 & 0.3337 & 0.1267 & 0.0000 & 0.5709 & 0.4795 \\
\hline CSF14 & 0.1333 & 0.2733 & 0.1267 & 0.1333 & 0.1258 & 0.1333 & 0.1250 & 0.3143 & 0.1222 & 0.1216 & 0.3337 & 0.1267 & 0.5091 & 0.0000 & 0.4500 \\
\hline CSF15 & 0.1042 & 0.1333 & 0.1267 & 0.3143 & 0.1258 & 0.1333 & 0.2889 & 0.0000 & 0.2800 & 0.1216 & 0.3634 & 0.1267 & 0.4205 & 0.5116 & 0.0000 \\
\hline
\end{tabular}


Table 8: Normalised direct relationship matrix for CSFs for effective adoption of sustainability initiatives in the automotive industry supply chain

\begin{tabular}{|c|c|c|c|c|c|c|c|c|c|c|c|c|c|c|c|}
\hline CSFs & CSF1 & CSF2 & CSF3 & CSF4 & CSF5 & CSF6 & CSF7 & CSF8 & CSF9 & CSF10 & CSF11 & CSF12 & CSF13 & CSF14 & CSF15 \\
\hline CSF1 & 0.0000 & 0.0379 & 0.0628 & 0.0379 & 0.0382 & 0.0412 & 0.0658 & 0.0412 & 0.0367 & 0.0364 & 0.0592 & 0.0628 & 0.0473 & 0.0476 & 0.0396 \\
\hline CSF 2 & 0379 & 0.0000 & 0.0628 & 0.0412 & 0.0663 & 0.0412 & 0.0378 & 0.0412 & 0.0160 & 0.0364 & 0.0515 & 0.0628 & 0.0628 & 0.0359 & 0.0628 \\
\hline CSF 3 & $\overline{0379}$ & 0.0379 & 0.0000 & 0.0412 & 0.0382 & 0.0412 & 0.0378 & 0.0412 & 0.0160 & 0.0159 & 0.0670 & 0.0385 & 0.0667 & 0.0359 & 0.0667 \\
\hline CSF4 & 0.0175 & 0.0175 & 0.0385 & 0.0000 & 0.0382 & 0.0412 & 0.0698 & 0.0175 & 0.0681 & 0.0159 & 0.0670 & 0.0166 & 0.0357 & 0.0670 & 0.0357 \\
\hline CSF5 & 0.0175 & 0.0412 & 0.0385 & 0.0175 & 0.0000 & 0.0412 & 0.0378 & 0.0175 & 0.0367 & 0.0159 & 0.0670 & 0.0166 & 0.0667 & 0.0359 & 0.0667 \\
\hline CSF6 & 0.0412 & 0.0175 & 0.0166 & 0.0412 & 0.0382 & 0.0000 & $\begin{array}{l}0.0378 \\
\end{array}$ & 0.0412 & 0.0367 & 0.0364 & $\begin{array}{l}0.0864 \\
\end{array}$ & 0.0385 & 0.0938 & 0.0670 & 0.0938 \\
\hline CSF7 & 0.0412 & 0.0175 & 0.0385 & 0.0412 & 0.0382 & 0.0175 & 0.0000 & 0.0175 & 0.0367 & 0.0159 & 0.0903 & 0.0166 & 0.0667 & 0.0670 & 0.0667 \\
\hline CSF8 & 0.0175 & 0.0175 & 0.0166 & 0.0175 & 0.0382 & 0.0412 & 0.0698 & 0.0000 & 0.0208 & 0.0677 & 0.0942 & 0.0385 & 0.0938 & 0.0670 & 0.0667 \\
\hline CSF9 & 0.0229 & 0.0229 & 0.0385 & 0.0175 & 0.0165 & 0.0412 & 0.0164 & 0.0229 & 0.0000 & 0.0873 & 0.0670 & 0.0166 & 0.0938 & 0.0942 & 0.0667 \\
\hline CSF10 & 0.0201 & 0.0258 & 0.0385 & 0.0412 & 0.0382 & 0.0175 & 0.0378 & 0.0412 & 0.0838 & 0.0000 & 0.0670 & 0.0166 & 0.0667 & 0.0359 & 0.0976 \\
\hline CSF11 & 0.0000 & 0.0175 & 0.0166 & 0.0412 & 0.0382 & 0.0175 & 0.0378 & 0.0412 & 0.0160 & 0.0364 & 0.0000 & 0.0385 & 0.0868 & 0.0670 & 0.0667 \\
\hline CSF12 & 0.0412 & 0.0412 & 0.0166 & 0.0175 & 0.0000 & 0.0412 & 0.0000 & 0.0412 & 0.0160 & 0.0364 & 0.0942 & 0.0000 & 0.0976 & 0.0942 & 0.0821 \\
\hline CSF13 & 0.0000 & 0.0175 & 0.0166 & 0.0175 & 0.0382 & 0.0175 & 0.0378 & 0.0175 & 0.0367 & 0.0159 & 0.0437 & 0.0166 & 0.0000 & 0.0748 & 0.0628 \\
\hline CSF14 & 0.0175 & 0.0358 & 0.0166 & 0.0175 & 0.0165 & 0.0175 & 0.0164 & 0.0412 & 0.0160 & 0.0159 & 0.0437 & 0.0166 & 0.0667 & 0.0000 & 0.0589 \\
\hline CSF15 & 0.0136 & 0.0175 & 0.0166 & 0.0412 & 0.0165 & 0.0175 & 0.0378 & 0.0000 & 0.0367 & 0.0159 & 0.0476 & 0.0166 & 0.0551 & 0.0670 & 0.0000 \\
\hline
\end{tabular}

Table 9: Total relationship matrix for CSFs for effective adoption of sustainability initiatives in the automotive industry supply chain

\begin{tabular}{|c|c|c|c|c|c|c|c|c|c|c|c|c|c|c|c|}
\hline CSFs & CSF1 & CSF2 & CSF3 & CSF4 & CSF5 & CSF6 & CSF7 & CSF8 & CSF9 & CSF10 & CSF11 & CSF12 & CSF13 & CSF14 & CSF15 \\
\hline CSF1 & 0.0301 & 0.0720 & 0.0990 & 0.0791 & 0.0807 & 0.0795 & 0.1134 & 0.0803 & 0.0798 & 0.0765 & 0.1444 & 0.0985 & 0.1410 & 0.1300 & 0.1275 \\
\hline CSF2 & 0.0656 & 0.0356 & 0.0985 & 0.0816 & 0.1067 & 0.0794 & 0.0878 & 0.0791 & 0.0608 & 0.0748 & 0.1359 & 0.0980 & 0.1535 & 0.1178 & 0.1478 \\
\hline CSF3 & 0.0612 & 0.0669 & 0.0337 & 0.0769 & 0.0758 & 0.0740 & 0.0826 & 0.0740 & 0.0551 & 0.0513 & 0.1390 & 0.0711 & 0.1458 & $\begin{array}{l}0.1091 \\
\end{array}$ & 0.1401 \\
\hline CSF4 & 0.0410 & 0.0458 & 0.0690 & 0.0348 & 0.0725 & 0.0715 & 0.1075 & 0.0499 & 0.1015 & 0.0507 & 0.1347 & 0.0465 & 0.1137 & 0.1339 & 0.1076 \\
\hline CSF5 & 0.0388 & 0.0661 & 0.0668 & 0.0506 & 0.0346 & 0.0693 & 0.0758 & 0.0474 & 0.0695 & 0.0474 & 0.1290 & 0.0459 & 0.1365 & 0.1003 & 0.1318 \\
\hline CSF6 & 0.0661 & 0.0518 & 0.0541 & 0.0818 & 0.0801 & 0.0381 & 0.0884 & 0.0785 & 0.0814 & 0.0762 & 0.1666 & 0.0741 & 0.1830 & 0.1501 & 0.1766 \\
\hline CSF7 & 0.0617 & 0.0466 & 0.0693 & 0.0752 & 0.0735 & 0.0495 & 0.0435 & 0.0502 & 0.0728 & 0.0496 & 0.1552 & 0.0479 & 0.1417 & 0.1346 & 0.1359 \\
\hline CSF8 & 0.0436 & 0.0507 & 0.0528 & 0.0591 & 0.0798 & 0.0755 & 0.1159 & 0.0387 & 0.0660 & 0.1038 & 0.1727 & 0.0725 & 0.1818 & 0.1472 & 0.1520 \\
\hline CSF9 & 0.0466 & 0.0542 & 0.0713 & 0.0563 & 0.0566 & 0.0733 & 0.0626 & 0.0597 & 0.0434 & 0.1200 & 0.1400 & 0.0499 & 0.1747 & 0.1652 & 0.1462 \\
\hline CSF10 & 0.0449 & 0.0567 & 0.0731 & 0.0789 & 0.0771 & 0.0536 & 0.0845 & 0.0746 & 0.1226 & 0.0404 & 0.1428 & 0.0502 & 0.1516 & 0.1153 & 0.1735 \\
\hline CSF11 & 0.0211 & 0.0435 & 0.0443 & 0.0707 & 0.0694 & 0.0461 & 0.0753 & 0.0684 & 0.0514 & 0.0650 & 0.0654 & 0.0636 & 0.1533 & 0.1289 & 0.1308 \\
\hline CSF12 & 0.0629 & 0.0708 & 0.0496 & 0.0556 & 0.0407 & 0.0733 & 0.0475 & 0.0766 & 0.0557 & 0.0723 & 0.1636 & 0.0352 & 0.1777 & 0.1659 & 0.1577 \\
\hline CSF13 & 0.0173 & 0.0385 & 0.0390 & 0.0425 & 0.0623 & 0.0401 & 0.0662 & 0.0405 & 0.0622 & 0.0404 & 0.0933 & 0.0377 & 0.0582 & 0.1224 & 0.1133 \\
\hline CSF14 & 0.0330 & 0.0547 & 0.0389 & 0.0424 & 0.0432 & 0.0402 & 0.0477 & 0.0626 & 0.0424 & 0.0405 & 0.0924 & 0.0393 & 0.1194 & 0.0509 & 0.1084 \\
\hline CSF15 & 0.0297 & 0.0377 & 0.0392 & 0.0643 & 0.0420 & 0.0396 & 0.0660 & 0.0241 & 0.0624 & 0.0395 & 0.0952 & 0.0376 & 0.1078 & 0.1145 & 0.0519 \\
\hline
\end{tabular}

\section{2}

URL: http://mc.manuscriptcentral.com/tppc E-mail: ppc@plymouth.ac.uk 
Table 10: Cause/effect parameters for CSFs for effective adoption of sustainability initiatives in the automotive industry supply chain

\begin{tabular}{|c|c|c|c|c|c|}
\hline CSFs for effective adoption of sustainability initiatives in supply chains & $\mathbf{R}$ & $\mathbf{D}$ & $\mathbf{R}+\mathbf{D}$ & R-D & Cause/Effect \\
\hline CSF1 & 1.4318 & 0.6635 & 2.0953 & 0.7682 & Cause \\
\hline CSF 2 & 1.4228 & 0.7916 & 2.2145 & 0.6312 & Cause \\
\hline CSF 3 & 1.2568 & 0.8986 & 2.1554 & 0.3583 & Cause \\
\hline CSF4 & 1.1807 & 0.9498 & 2.1304 & 0.2309 & Cause \\
\hline CSF5 & 1.1097 & 0.9951 & 2.1048 & 0.1146 & Cause \\
\hline CSF6 & 1.4469 & 0.9028 & 2.3496 & 0.5441 & Cause \\
\hline CSF7 & 1.2072 & 1.1647 & 2.3719 & 0.0425 & Cause \\
\hline CSF8 & 1.4122 & 0.9048 & 2.3170 & 0.5074 & Cause \\
\hline CSF9 & 1.3201 & 1.0269 & 2.3470 & 0.2932 & Cause \\
\hline CSF10 & 1.3398 & 0.9485 & 2.2883 & 0.3913 & Cause \\
\hline CSF11 & 1.0972 & 1.9701 & 3.0673 & -0.8729 & Effect \\
\hline CSF12 & 1.3051 & 0.8682 & 2.1732 & 0.4369 & Cause \\
\hline$\sqrt{20}$ & 0.8740 & 2.1397 & 3.0137 & -1.2657 & Effect \\
\hline 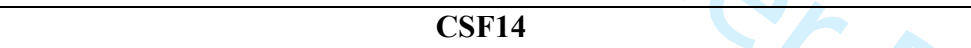 & 0.8560 & 1.8861 & 2.7420 & -1.0301 & Effect \\
\hline CSF15 & 0.8516 & 2.0015 & 2.8530 & -1.1499 & Effect \\
\hline
\end{tabular}




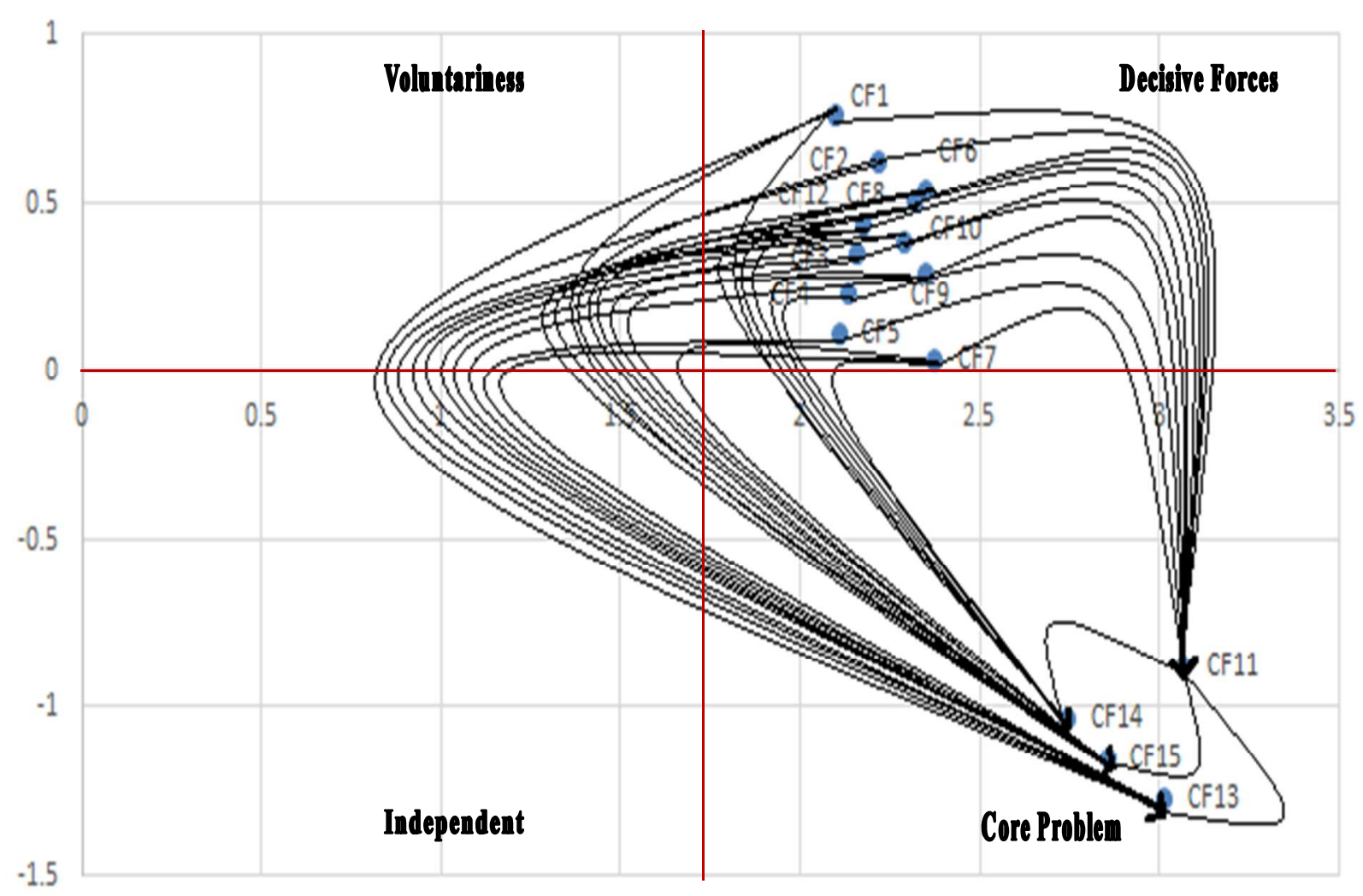

\section{$\mathrm{X}$ axis- ( $\mathrm{R}+\mathrm{D})$; $\mathrm{Y}$ axis- (R-D)}

Figure 2: Diagraph represents causal relationship among CSFs for effective adoption of sustainability initiatives in supply chain

\section{Sensitivity Analysis}

Sensitivity analysis assesses the variation in cause-effect relationship by giving different weights to industrial experts. The sensitivity analysis can also check the effect of human bias on the outcome of the study. As a further step, sensitivity analysis also provides methodological generalizability perspectives to the results. Sensitivity analysis has been performed by giving major weights for randomly selected 8 experts independently, keeping identical weights for the others as illustrated in Table 11.

Table 11: Weights assigned for eight experts during sensitivity analysis

\begin{tabular}{l|c|c|c|c|c|c|c|c}
\hline Run & Expert 1 & Expert 2 & Expert 3 & Expert 4 & Expert 5 & Expert 6 & Expert 7 & Expert 8 \\
\hline Sensitivity Run 1 & 0.3 & 0.1 & 0.1 & 0.1 & 0.1 & 0.1 & 0.1 & 0.1 \\
\hline Sensitivity Run 2 & 0.1 & 0.3 & 0.1 & 0.1 & 0.1 & 0.1 & 0.1 & 0.1 \\
\hline Sensitivity Run 3 & 0.1 & 0.1 & 0.3 & 0.1 & 0.1 & 0.1 & 0.1 & 0.1 \\
\hline Sensitivity Run 4 & 0.1 & 0.1 & 0.1 & 0.3 & 0.1 & 0.1 & 0.1 & 0.1 \\
\hline Sensitivity Run 5 & 0.1 & 0.1 & 0.1 & 0.1 & 0.3 & 0.1 & 0.1 & 0.1 \\
\hline
\end{tabular}




\begin{tabular}{l|l|l|l|l|l|l|l|l}
\hline Sensitivity Run 6 & 0.1 & 0.1 & 0.1 & 0.1 & 0.1 & 0.3 & 0.1 & 0.1 \\
\hline Sensitivity Run 7 & 0.1 & 0.1 & 0.1 & 0.1 & 0.1 & 0.1 & 0.3 & 0.1 \\
\hline Sensitivity Run 8 & 0.1 & 0.1 & 0.1 & 0.1 & 0.1 & 0.1 & 0.1 & 0.3 \\
\hline
\end{tabular}

In the sensitivity analysis run 1; Expert 1 has highest weightage (0.3) and other experts have equal weightage (0.1). The results of sensitivity analysis for all the runs are shown in Table 12 .

Table 12: Sensitivity analysis of CSFs for effective adoption of sustainability initiatives in the supply chains

\begin{tabular}{|c|c|c|c|c|c|c|c|c|c|c|c|c|}
\hline \multirow{2}{*}{ CSFs } & \multicolumn{3}{|c|}{ Sensitivity Run 1} & \multicolumn{3}{|c|}{ Sensitivity Run 2} & \multicolumn{3}{|c|}{ Sensitivity Run 3} & \multicolumn{3}{|c|}{ Sensitivity Run 4} \\
\hline & $\mathbf{R}+\mathbf{D}$ & R-D & Rank & $\mathbf{R}+\mathbf{D}$ & R-D & Rank & $\mathbf{R}+\mathbf{D}$ & R-D & Rank & $\mathbf{R}+\mathbf{D}$ & R-D & Rank \\
\hline CSF1 & 2.1084 & 0.7837 & 1 & 2.1243 & 0.7600 & 1 & 2.0794 & 0.7616 & 1 & 2.1378 & 0.7853 & 1 \\
\hline CSF 2 & 2.2459 & 0.6601 & 2 & 2.2164 & 0.6347 & 2 & 2.2052 & 0.6015 & 2 & 2.2658 & 0.6193 & 2 \\
\hline CSF 3 & 2.1783 & 0.3562 & 7 & 2.1473 & 0.3651 & 7 & 2.1424 & 0.3547 & 7 & 2.1981 & 0.3646 & 7 \\
\hline CSF4 & 2.1750 & 0.2455 & 9 & 2.1277 & 0.2289 & 9 & 3.0000 & 0.2278 & 9 & 2.1679 & 0.2331 & 9 \\
\hline CSF5 & 2.1557 & 0.1222 & 10 & 2.1010 & 0.1138 & 10 & 2.0881 & 0.1100 & 10 & 2.1410 & 0.1165 & 10 \\
\hline CSF6 & 2.3798 & 0.5329 & 3 & 2.3279 & 0.5234 & 3 & 2.3371 & 0.5465 & 3 & 2.3975 & 0.5612 & 3 \\
\hline CSF7 & 2.4343 & 0.0498 & 11 & 2.3485 & 0.0186 & 11 & 2.3460 & 0.0540 & 11 & 2.4160 & 0.0449 & 11 \\
\hline CSF8 & 2.3493 & 0.5023 & 4 & 2.3101 & 0.5069 & 4 & 2.2957 & 0.5064 & 4 & 2.3559 & 0.5186 & 4 \\
\hline CSF9 & 2.3661 & 0.2688 & 8 & 2.3557 & 0.3045 & 8 & 2.3376 & 0.3033 & 8 & 2.3798 & 0.2980 & 8 \\
\hline CSF10 & 2.3293 & 0.3933 & 6 & 2.2992 & 0.4014 & 6 & 2.2829 & 0.3991 & 6 & 2.3230 & 0.3987 & 6 \\
\hline CSF11 & 3.1033 & -0.8645 & 12 & 3.0266 & -0.8490 & 12 & 3.0472 & -0.8853 & 12 & 3.1304 & $\begin{array}{c}-0.8910 \\
\end{array}$ & 12 \\
\hline CSF12 & 2.2262 & 0.4469 & 5 & 2.1708 & 0.4304 & 5 & 2.1425 & 0.4141 & 5 & 2.2224 & 0.4496 & 5 \\
\hline CSF13 & 3.0777 & -1.2472 & 15 & 2.9930 & -1.2796 & 15 & 3.0016 & -1.2539 & 15 & 3.0574 & -1.2676 & 15 \\
\hline CSF14 & 2.8396 & -1.0780 & 13 & 2.7551 & -1.0173 & 13 & 2.7149 & -0.9891 & 13 & 2.7906 & -1.0677 & 13 \\
\hline CSF15 & 2.9460 & -1.1722 & 14 & 2.8436 & -1.1418 & 14 & 2.8444 & -1.1507 & 14 & 2.9002 & -1.1633 & 14 \\
\hline \multirow{2}{*}{ CSFs } & \multicolumn{3}{|c|}{ Sensitivity Run 5} & \multicolumn{3}{|c|}{ Sensitivity Run 6} & \multicolumn{3}{|c|}{ Sensitivity Run 7} & \multicolumn{3}{|c|}{ Sensitivity Run 8} \\
\hline & $\mathbf{R}+\mathbf{D}$ & R-D & Rank & $\mathbf{R}+\mathbf{D}$ & R-D & Rank & $\mathbf{R}+\mathbf{D}$ & R-D & Rank & $\mathbf{R}+\mathbf{D}$ & R-D & Rank \\
\hline CSF1 & 2.0907 & 0.7547 & 1 & 2.0532 & 0.7427 & 1 & 2.0612 & 0.7756 & 1 & 2.0600 & 0.7489 & 1 \\
\hline CSF 2 & 2.2106 & 0.6237 & 2 & 2.1851 & 0.6255 & 2 & 2.1528 & 0.6228 & 2 & 2.1887 & 0.6351 & 2 \\
\hline CSF 3 & 2.1425 & 0.3540 & 7 & 2.1279 & 0.3526 & 7 & 2.1250 & 0.3496 & 7 & 2.1437 & 0.3510 & 7 \\
\hline CSF4 & 2.1126 & 0.2264 & 9 & 2.0935 & 0.2202 & 9 & 2.0982 & 0.2235 & 9 & 2.1139 & 0.2214 & 9 \\
\hline CSF5 & 2.0870 & 0.1123 & 10 & 2.0714 & 0.1097 & 10 & 2.0722 & 0.1097 & 10 & 2.0894 & 0.1075 & 10 \\
\hline CSF6 & 2.3364 & 0.5454 & 3 & 2.3180 & 0.5380 & 3 & 2.3170 & 0.5396 & 3 & 2.3347 & 0.5403 & 3 \\
\hline CSF7 & 2.3557 & 0.0423 & 11 & 2.3388 & 0.0389 & 11 & 2.3405 & 0.0398 & 11 & 2.3598 & 0.0358 & 11 \\
\hline CSF8 & 2.2959 & 0.5046 & 4 & 2.2923 & 0.4964 & 4 & 2.2764 & 0.4995 & 4 & 2.3119 & 0.5007 & 4 \\
\hline CSF9 & 2.3371 & 0.3022 & 8 & 2.2709 & 0.3086 & 8 & 2.3104 & 0.2865 & 8 & 2.3110 & 0.3258 & 8 \\
\hline CSF10 & 2.2824 & 0.3978 & 6 & 2.2030 & 0.3996 & 6 & 2.2543 & 0.3830 & 6 & 2.2540 & 0.3678 & 6 \\
\hline CSF11 & 3.0639 & -0.8819 & 12 & 3.0217 & -0.8580 & 12 & 3.0389 & -0.8779 & 12 & 3.0379 & -0.8609 & 12 \\
\hline CSF12 & 2.1559 & 0.4266 & 5 & 2.1505 & 0.4323 & 5 & 2.0927 & 0.4710 & 5 & 2.1210 & 0.4832 & 5 \\
\hline CSF13 & 3.0105 & -1.2632 & 15 & 2.9761 & -1.2692 & 15 & 2.9805 & -1.2484 & 15 & 2.9611 & -1.2851 & 15 \\
\hline CSF14 & 2.7136 & -1.0127 & 13 & 2.6846 & -1.0180 & 13 & 2.6822 & -1.0181 & 13 & 2.6992 & -1.0152 & 13 \\
\hline CSF15 & 2.8220 & -1.1321 & 14 & 2.8169 & -1.1194 & 14 & 2.7926 & -1.1560 & 14 & 2.8125 & -1.1564 & 14 \\
\hline
\end{tabular}

Next, we examined the causal relationship among the CSFs, and showed the three most important causal factors (CSF1, CSF2 and CSF6) in sustainability practice implementation in the supply chain (see Figure 3). 


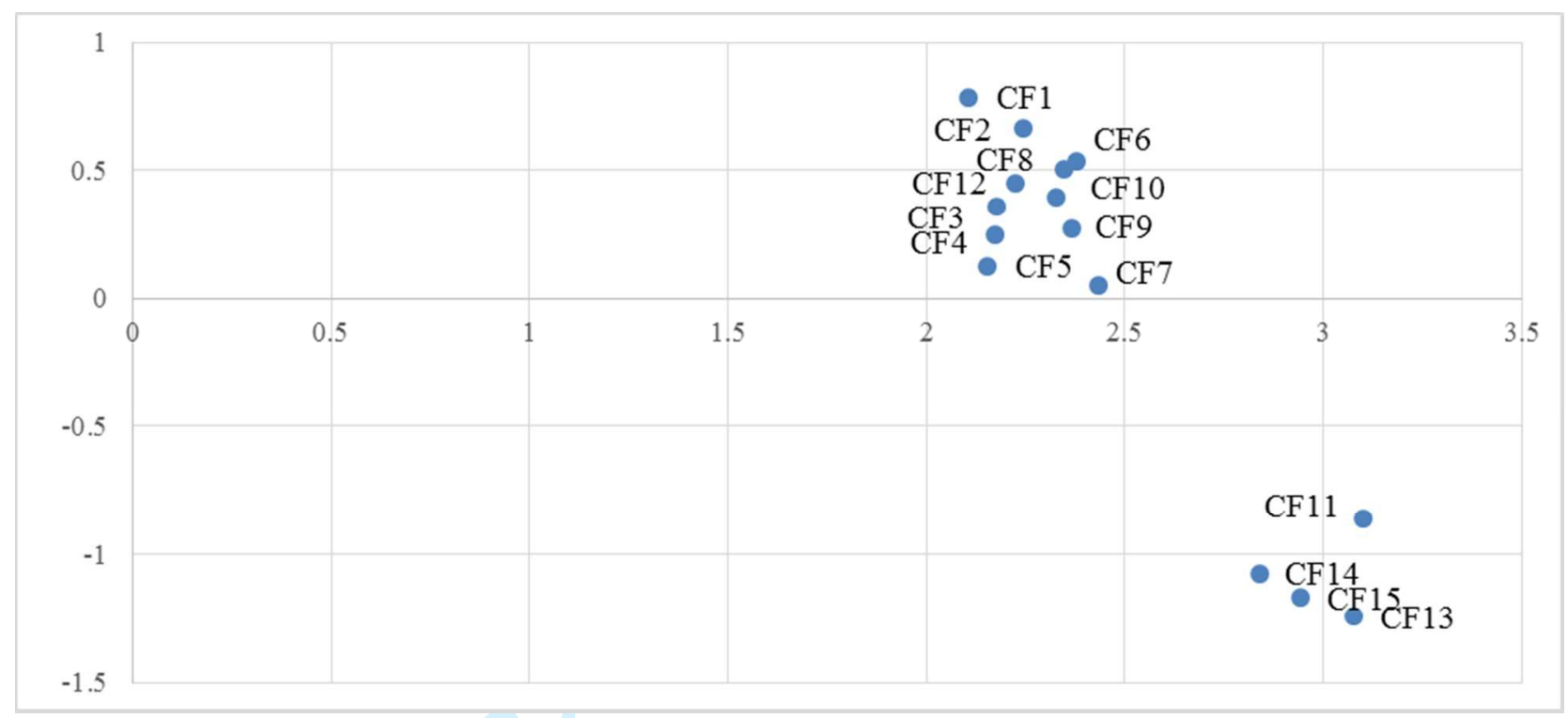

Figure 3: Causal relationship illustration among CSFs for effective adoption of sustainability initiatives in supply chain obtained from sensitivity analysis run 1

Similarly, all sensitivity analysis runs have been performed and causal relationships among the CSFs for run 2 to run 8 are evaluated as shown in Appendix-C.

The sensitivity analysis shows that CSF1, CSF2 and CSF6 are the three most important causal factors in all runs and CSF11, CSF14, CSF15 and CSF13 are effect factors in the entire runs. The performed sensitivity analysis determined whether the decision making process has been affected by different weightage values assigned to decision makers. According to the sensitivity analysis results, there is almost same ranking order for the cause/effect factors in each case, accepting slight order discrepancies.

To this support, cause and effect diagrams also showed slight variations in the causal relationship on the diagrams mapped in Appendix-C (Figures B1-B7). Hence, it can be inferred that proposed framework is robust enough to deal with human bias and vagueness in data.

\section{Discussions of Findings}

According to the dataset (R-D) values, eleven CSFs for successful adoption of sustainability initiatives in the supply chain namely Government Legalisation (CSF1) > Top Management Support (CSF2) > Technology development and process innovation (CSF6) > Trainings (CSF8) $>$ Customer Involvement and Encouragement $(\mathrm{CSF} 12)>$ Reverse Logistics and Waste 
Minimisation $($ CSF10) $>$ Ecological Considerations in Organisations' Policies and Missions $(\mathrm{CSF} 3)>$ Green Design and Purchasing $(\mathrm{CSF} 9)>$ Societal Considerations $(\mathrm{CSF} 4)>$ Supply Chain Members' Collaborations (CSF5) > Communication and Information Technology (CSF7) have been classified into the cause group CSFs. In addition, four CSFs namely Ethical and Safe Practices $(\mathrm{CSF} 11)>$ Community Welfare and Development (CSF13) $>$ Economic Considerations (CSF14) > Competitiveness and Brand Image Considerations (CSF15) have been classified into the effect group. The correlation between the CSFs are given in the Figure 2, which shows that CSF1 exhibits the highest correlation with other CSFs; because Government Legalisation towards sustainable initiatives in the supply chain is necessary to implement sustainable practices and their concern also influence other stakeholder's of supply chain.

In addition, the identified factors for successful sustainability initiatives in supply chains have been mapped into four quadrants (decisive, voluntariness, independent and core problems) and present a visual structure to decision maker.

Quadrant I drivers have the highest relation and prominence, referring to the maximum interaction influence on other CSFs. With respect to this, eleven drivers (CSF1, CSF2, CSF3, CSF4, CSF5, CSF6, CSF7, CSF8, CSF9, CSF10 and CSF12) fall into the decisive region. It means that these eleven factors play decisive roles in incorporating sustainability initiatives in automotive industry supply chains. These eleven CSFs also belong to the cause group factors. Among the entire cause group CSFs, 'Government Legalisation (CSF1)' has the maximum (RD) score of 0.7682 , which signifies that CSF1 has highest influence on the whole system. However, its $(\mathrm{R}+\mathrm{D})$ score (2.0953) is relatively small, which could be justified by the fact that government regulations can affect other factors but receive moderately small influence in return. To this support, many researchers have shown the importance of government legalisation and regulatory norms in implementing sustainability in supply chains (Ageron, et al., 2012; Giunipero et al., 2012; Walker and Jones, 2012; Al Zaabi et al., 2013). Government authorities may play a critical role (command and control) in effective adoption of sustainability initiatives in supply chains e.g. carbon tax and subsidising etc. (Gupta and Palsule-Desai, 2011). The automotive companies agreed that government legalisations and regulatory norms enforcement in this direction may be threshold point for implementing sustainable initiatives to Indian supply chains. The second highest critical success factor in the (R-D) column is the 'Top Management Support (CSF2)', with a score of 0.6312 , which also has reasonable power to affect other factors 
as given by influential impact index $(\mathrm{R})$ value equal to 1.4228 . Top management supports play a very influential role in inspiring business organisations and are responsible for the business organisations' sustainable initiatives (Giunipero et al., 2012). The sustainability practices adoption is still considered as a costly affair in Indian automotive manufacturing organisations. Moreover, the understanding of its significant is usually neglected. Therefore, the top management must be sensitised so that they should inject a strong culture that eventually assist organisation in facilitates maximum freedom and leads employee to make establishing an efficient system and method to present environmental improvements without any intervention. 'Technology development and process innovation (CSF6)', with (R-D) score of 0.5441 has third ranking signifying its importance, but at the same time having the highest influential impact driver (R) equal to 1.4469 on the overall system in enhancing the supply chain sustainability. The selection of appropriate pollution prevention and cleaner technologies will help business organisations to achieve sustainability goals in their supply chain (Almeida et al., 2013, 2015). Further, for sustainable business gains, managers and practitioners should seek to achieve supply chain sustainability from system perspective, which requires process innovation in terms of development of lean, green, circular, JIT, Poka-yoke based concepts on operational, tactical and strategic levels (Piercy and Rich, 2015). According to a World Bank report, India is among the world's leading innovation players in the automobile parts and assembly sectors of the manufacturing industry. Mahindra \& Mahindra, a private sector automobile company in India, adopted innovation in their manufacturing process at various levels, thus enabling the company to lower production costs though saving the material and energy (sustainability) while developing its multi-utility vehicle "Scorpio". Likewise, next CSF 'Training (CSF8)', with an (R-D) score of 0.5074 , and helps in educating supply chain members in the use of innovative technologies, processes and effective use of resources, fostering sustainable practices in supply chains (Hsu et al., 2016). This finding also echo the results obtained in Spanish automotive industry by Sarkis et al. (2010). 'Customer Involvement and Encouragement (CSF12)' (with (R-D) score of 0.4369) plays a significant role in understanding and responding to customers' purchasing behaviour. Therefore, information on customers' needs should be regularly collected and evaluated (Bask et al., 2013) and value creation for customers, which will help business organisations to achieve sustainable competitive advantage (Ageron et al., 2012). Next, 'Reverse Logistics and Waste Minimisation (CSF10)' (with (R-D) score of 0.3913) is 
important with an objective of minimising waste and increasing the amount of product materials recovered from the waste (Gunasekaran and Spalanzani, 2012). Maruti Suzuki India Limited is an automobile manufacturer in India has started to buying back old used cars. The factor 'Ecological Considerations in Organisations' Policies and Missions (CSF3)' has an (R-D) score of 0.3583. Ecological considerations in organisations' policies and mission will provide a proactive stance towards the environment and in improving ecological efficiency (Gold et al., 2013). Next to this, the factor 'Green Design and Purchasing (CSF9)' has an (R-D) score of 0.3583, showing its importance. In addition, its $(\mathrm{R}+\mathrm{D})$ score (equals to 2.3470 ) is comparatively high, meaning that green design and purchasing policies are not only influencing other factors but receive influence in return from other factors in sustainability adoption in supply chains (Tseng et al., 2013). Tata Motors, the world's fifth largest commercial vehicle manufacturer, is extending its basket of designing environmentally friendly vehicles. Tata Motors also has a joint venture with Marcopolo S.A. of Brazil, one of the largest bus body manufactures in the world, for safety, \& fuel efficiency. In October 2016, Ashok Leyland Ltd. unveiled its Circuit series electric bus - the country's first such indigenously made vehicle. According to the Government of India's Faster Adoption and Manufacturing of (Hybrid) and Electric Vehicles (FAME) scheme, this gives subsidies to such initiatives to put seven million electric and hybrid vehicles on road by 2020. This is in line with our finding that government role is given highest priority in critical CSFs. Following this, the factor Societal Considerations (CSF4) is also a decisive factor in an effective adoption and implementation of sustainability in business. This factor has an (RD) score of 0.2309 , higher than the Supply Chain Members' Collaborations (CSF5) > Communication and Information Technology (CSF7) (R-D) score. The literature suggests that social aspects are generally missing or understood in an unusual way (Seuring, 2013), and socially responsible practices can positively influence sustainability initiatives in supply chains (Walker and Jones, 2012). Lastly, in the cause group, the factors 'Supply Chain Members' Collaborations (CSF5) and Communication and Information Technology (CSF7) come with (RD) scores of 0.1146 and 0.0425 respectively. Supply Chain Members' Collaborations aims to collaboratively develop new technologies, processes and sustainable products (Beske et al., 2014). Communication and information technology support information complexity, proliferation, diffusion, and velocity (Subramanian and Gunasekaran, 2015) may play critical role in developing capabilities on sustainability issues; and achieve sustained competitive 
advantage (Dao et al., 2011). The working on these factors will assist managers to formulate policies for implementation of the successful implementation of sustainability in a supply chain context.

Quadrant II has lower prominence but high relation, and is known as voluntariness. After focusing decisive group CSFs, the voluntariness area's CSFs must be attempted. In the present research, no factor is located in this quadrant; hence, none of the CSFs is treated as a follow-up factors needs to be considered to be incorporated in sustainability initiatives in supply chains.

Quadrant III (independent) indicates low prominence and relation; and less interaction within the system. None of the factors fall into the independent area.

Quadrant IV represents the core problems (high prominence and low relation) that are required to be solved. Factors in this quadrant have a tendency to be effortlessly influenced by other factors. It means that these factors are actually core problems, and may not directly improve the system, but should be improved by other factors e.g. decisive group factors. There are four factors in this group, which are the effect group factor as well. In all the drivers, 'Ethical and Safe Practices (CSF11)' obtain the highest (R-D) score of -0.8729 , which suggests that this factor receives the least impact. The factor 'CSF11' is among the top factors according to an (R+D) a score of 3.0673 means the significance of this factor. As the public is becoming aware of environmental and societal issues, automotive companies in India have been facing pressure from their customers to produce high-quality, safe and environmentally friendly products (Zailani et al., 2012; Wilhelm et al., 2016). The other factors follow the sequence of increasing order of priority list in the effect group, include 'Economic Considerations (CSF14)' with an (R-D) a score of 1.0301, 'Competitiveness and Brand Image Considerations (CSF15)'with an (R-D) a score of 1.1499, 'Community Welfare and Development (CSF13)' with an (R-D) a score of -1.2657. One main reason behind sustainable initiatives in under study supply chains is management's desire for achieving a high brand image in the market (Ageron et al., 2012). Sustainability initiatives in supply chains can certainly influence a business organisation's profitability, performance competitive advantage and enhanced brand image (Carter and Rogers, 2008; Golicic and Smith, 2013). That means social issues (human health \& safety and community welfare \& development) are major concerns for sustainability initiatives in supply chains of Indian automotive companies (Fabbe-Costes et al., 2014). 
Notably, the experts showed an agreement with the findings. However, it is difficult to state that above findings will be strictly applicable to other industry sector in the developing economy like India. Thus, the managers are recommended to adopt the proposed framework with marginal modifications to establish the causal relationship among the identified CSFs of developing sustainability in supply chains.

\subsection{Policy recommendation and implication for managers in implementing sustainability initiatives in supply chains in India}

In this section, several policy recommendation and implications for implementing sustainability initiatives in automotive sector supply chain from Indian context is provided. Sustainability initiatives have received great attention in achieving ecological, social and economic benefits for supply chain practitioners and researchers (Gopalakrishnan et al., 2012). This leads to incredible savings in terms of resources, money and has a potential to generate plenty of employment opportunities. However, in developing economy, such as India, in order to implement sustainability initiatives, the most critical success factor is Government Legalisation. The current level of implementation of sustainability initiative is India is at nascent stage as compared to developed countries, such as European countries and USA. India follows the traditional command-and-control mechanism whereas, European Union and United States follows marketbased regulatory mechanism. Unlike market-based approaches, the traditional command-andcontrol regulatory mechanism provides no incentive for a business organisation if it keeps its level of pollution and or carbon emissions below the amount authorised by regulation (Kayden, 1991). Therefore, government legalisation becomes a binding constraint for Indian automotive sector and plays a crucial role for the success implementation of sustainability practices. Moreover, an appropriate explanation of the currently low level of sustainability adoption can be explained by the fact that the regulatory pressures can easily be overcome using symbolic or reluctant efforts as the reduction targets are not very high.

The developing economy, such as India is also more sensitive to additional overheads due to ecofriendly activities as compared to the advanced economy. The anticipated payback period is crucial in sustainability adoption. Creating new resources via public funds and organisational financial budgets might be troublesome in India. Government and management support could ease the investment provisions in the domain of sustainability and encourage research for 
sustainability implementation by providing subsidies and tax credit initiatives (Gupta and Palsule-Desai, 2011). In Indian context, top management support is essential for any business organisation in strategy and vision development, and to assign sufficient human resources and technological support for effective adoption of sustainability (Wittstruck and Teuteberg, 2012).

Top management should support technological advancement and process innovation for business sustainability in Indian scenario. The advancement and innovations in technology and processes not only leads to lower environmental pollution but also higher economic performance. For example, process improvement using lean reduces waste and polluting which leads to win-win situation. This will allow in reducing the related problematic issues in developing sustainable supply chains, understand trade-offs in sustainable operations during design and implementation in practice (Lii and Kuo, 2016). Organisations in automotive sector in India should develop a national strategy for developing the expertise of people in the sustainable manufacturing background. Managers can arrange training sessions, apprenticeship programs with in depth knowledge of sustainability oriented practices (Mangla et al., 2013).

The involvement of customer in value chains is significant for business sustainability. In India, customer awareness and active participation can push industries in automotive sector to adopt sustainable practices. Management should collaborate with their customers in effective SSCM adoption. The degree to which top management are willing to implement sustainability focused initiatives in Indian automotive industry context is usually depend on cost effectiveness. Less understanding on the advantages of the business sustainability hampers its adoption in India (Luthra et al., 2015). In India, management generally considers resource efficient operations as an additional financial burden on their businesses. The government should take responsibility and provide guidelines to automotive sector organisations in exploring enormous opportunities, such as waste management, community development, resources conservations, pollution prevention and control, economic growth and development, in Indian context. Low technical competence may inhibit Indian automotive sector from capitalising on business sustainable. In this case, higher infrastructure and resources facilities can assist Indian managers in promoting economic, ecological and societal considerations in value chains. In addition, green design and purchasing decisions would help Indian automotive managers in achieving an environmentally efficient system and endorse green marketing (Brindley and Oxborrow, 2014). This will further 
improve the competitiveness of Indian automotive sector and emerges them as the global players in the market.

In a developing country like India, efficient access to information and visibility of the entire value chain is crucial in business sustainability (Prakash and Barua, 2015). Sustainable consumption and production and other advanced technology driven sustainable business models needs to be developed. Robust and flexible strategies need to be modelled to track the resource flows to assist automotive companies to minimise their process waste. There is a need to change the behaviour of customer to manage the substantial amount of waste generated at consumer level in India. Suitable end of life treatment must be provided for the used products in automotive sector. Reverse logistics, is very useful in such situations, so as it allows automotive company managers to capture the value of products and material through an infinite loop of reuse. In case of developing nation like India, managers need to strengthen their organisational capabilities in initiating reverse logistics initiatives, such as reuse, recycle and remanufacturing (Mangla et al., 2016). Thus, automotive companies should follow an innovative approach in terms of collecting and exchanging information, investing in Research \& Development, disseminating good practices, promoting supplier-organisation-customer collaboration.

\section{Conclusions, Limitations and Future Works}

Sustainability has been attaining significant attention from practitioners and researchers in formulation of business strategies from a supply chain context. At the same time, it has also been seen that the adoption of sustainability is difficult for the organisations, especially in developing nations, such as India due to the existence of various significant factors related to finance, management, government regulations etc. In this work, an effort is made to incorporate effective sustainability initiatives by uncovering the relevant CSFs in supply chains in Indian context from the system perspectives.

In this work, we employed a grey based DEMATEL technique to examine the influential and influenced interactions among the sustainability oriented CSFs. The proposed research framework is applied to a multiple case study of three automotive companies in India. Total 15 CSFs related to effective sustainability initiatives based on the literature and expert's inputs were listed. Based on Grey-DEMATEL application that uncovers the causal relation among the identified factors, cause and effect group are revealed. The factors CSF1, CSF2, CSF6, CSF8, 
CSF12, CSF10, CSF3, CSF9, CSF4, CSF5 and CSF7 are classified into the cause group, which needs a greater managerial attention to have the desired sustainable initiatives in supply chains. In addition, the factors CSF11, CSF13, CSF14 and CSF15 classify into the effect group, and have to be worked upon to enhance the sustainable initiative decisions success rate. However, continuous supervision is suggested on the recognised CSFs and the relevant activities to attain success in the implementation of sustainability aspects from the industry supply chain context in India.

This study has few limitations as well. The detection of the sustainability focused CSFs could be challenging for future studies for two reasons. Firstly, as the developing country like India is more on the track of growth, some factors which have highest influential power may become insignificant in future, and some factor which has eliminated (Investment Recovery) may become significant once organisations matured in sustainability. Secondly, due to higher rate of technological innovations, the industry may witness some breakthrough innovations which may change the entire competitive, economic, environmental and social landscape. Next, this work uses expert's opinion. To deal with this, the procedure needs to be carried out very carefully. This work uses multiple case study approach. Thus, the sample size may be increased and empirical study with higher sample size may be conducted to examine how the CSFs influence the definite objective of sustainability initiatives in a supply chain scenario. The developed framework is applied to Indian context. Thus, we may apply the framework with marginal modifications in other developing countries and results may be compared in future studies.

\section{References}

Ageron, B., A. Gunasekaran, and A. Spalanzani. 2012. "Sustainable Supply Management: An Empirical Study." International Journal of Production Economics 140 (1): 168-182.

Ahi, P., and C. Searcy. 2015. "An Analysis of Metrics Used to Measure Performance in Green and Sustainable Supply Chains." Journal of Cleaner Production 86: 360-377.

Al Zaabi, S., N. Al Dhaheri, and A. Diabat. 2013. "Analysis of Interaction between the Barriers for the Implementation of Sustainable Supply Chain Management." The International Journal of Advanced Manufacturing Technology 68 (1-4): 895-905.

Almeida, C. M. V. B., F. Agostinho, B. F. Giannetti, and D. Huisingh. 2015. "Integrating Cleaner Production into Sustainability Strategies: An Introduction to This Special Volume." Journal of Cleaner Production 96: 1-9.

Almeida, C. M. V. B., S. H. Bonilla, B. F. Giannetti, and D. Huisingh. 2013. "Cleaner Production Initiatives and Challenges for a Sustainable World: An Introduction to This Special Volume." Journal of Cleaner Production 47: 
$1-10$.

Amindoust, A., S. Ahmed, A. Saghafinia, and A. Bahreininejad. 2012. "Sustainable Supplier Selection: A Ranking Model Based on Fuzzy Inference System." Applied Soft Computing 12 (6): 1668-1677.

Andiç, E., Ö Yurt, and T. Baltacioğlu. 2012. "Green Supply Chains: Efforts and Potential Applications for the Turkish Market." Resources, Conservation and Recycling 58: 50-68.

Arora, R (2016). Government to issue notification for implementation of Bharat Stage (BS) VI emission norms.

Available at: http://economictimes.indiatimes.com/articleshow/54382376.cms? utm source $=$ contentofinterest \&utm_medium $=$ text\&utm_campaign $=$ cppst $($ Accessed on Sep 17, 2016).

Ashby, A., M. Leat, and M. Hudson-Smith. 2012. "Making Connections: A Review of Supply Chain Management and Sustainability Literature." Supply Chain Management: An International Journal 17 (5): 497-516.

Azadi, M., M. Jafarian, R. F. Saen, and S. M. Mirhedayatian. 2015. "A New Fuzzy DEA Model for Evaluation of Efficiency and Effectiveness of Suppliers in Sustainable Supply Chain Management Context." Computers \& Operations Research 54: 274-285.

Bai, C., and J. Sarkis. 2010. "Integrating Sustainability into Supplier Selection with Grey System and Rough Set Methodologies." International Journal of Production Economics 124 (1): 252-264.

Bai, C., and J. Sarkis. 2013. "A Grey-Based DEMATEL Model for Evaluating Business Process Management Critical Success Factors." International Journal of Production Economics 146 (1): 281-292.

Bai, C., and J. Sarkis. 2014. "Determining and Applying Sustainable Supplier Key Performance Indicators." Supply Chain Management: An International Journal 19 (3): 275-291.

Bask, A., M. Halme, M. Kallio, and M. Kuula. 2013. "Consumer Preferences for Sustainability and Their Impact on Supply Chain Management: The Case of Mobile Phones." International Journal of Physical Distribution \& Logistics Management 43 (5/6): 380-406.

Baumann, C. E., and B. V. Genoulaz. 2014. "A Framework for Sustainable Performance Assessment of Supply Chain Management Practices." Computers \& Industrial Engineering 76: 138-147.

Beske, P., A. Land, and S. Seuring. 2014. "Sustainable Supply Chain Management Practices and Dynamic Capabilities in the Food Industry: A Critical Analysis of the Literature." International Journal of Production Economics 152: 131-143.

Boer*, H., F. Gertsen, R. Kaltoft and J .S. Nielsen 2005. Factors affecting the development of collaborative improvement with strategic suppliers. Production Planning \& Control, 16(4): 356-367.

Bose, I., and R. Pal. 2012. "Do Green Supply Chain Management Initiatives Impact Stock Prices of Firms?." Decision Support Systems 52 (3): 624-634.

Brandenburg, M., K. Govindan, J. Sarkis, and S. Seuring. 2014. "Quantitative Models for Sustainable Supply Chain Management: Developments and Directions." European Journal of Operational Research 233 (2): 299-312.

Brindley, C., and L. Oxborrow. 2014. "Aligning the Sustainable Supply Chain to Green Marketing Needs: A Case Study." Industrial Marketing Management 43 (1): 45-55.

Büyüközkan, G., and G. Çif̧ci. 2011. "A Novel Fuzzy Multi-Criteria Decision Framework for Sustainable Supplier Selection with Incomplete Information." Computers in Industry 62 (2): 164-174. 
Büyüközkan, G., and G. Çifçi. 2012. "A Novel Hybrid MCDM Approach Based on Fuzzy DEMATEL, Fuzzy ANP and Fuzzy TOPSIS to Evaluate Green Suppliers." Expert Systems with Applications 39 (3): 3000-3011.

Carter, C. R., and D. S. Rogers. 2008. "A Framework of Sustainable Supply Chain Management: Moving toward New Theory." International Journal of Physical Distribution \& Logistics Management 38 (5): 360-387.

Chaabane, A., A. Ramudhin, and M. Paquet. 2011. "Designing Supply Chains with Sustainability Considerations." Production Planning \& Control 22 (8): 727-741.

Chaabane, A., A. Ramudhin, and M. Paquet. 2012. "Design of Sustainable Supply Chains under the Emission Trading Scheme." International Journal of Production Economics 135 (1): 37-49.

Chen, T. B., and L. T. Chai. 2010. "Attitude towards the Environment and Green Products: Consumers' Perspective." Management Science and Engineering 4 (2): 27-39.

Chuang, S. P. and C. L. Yang 2014. Key success factors when implementing a green-manufacturing system. Production Planning \& Control, 25(11): 923-937.

Dao, V., I. Langella and J. Carbo. 2011. "From Green to Sustainability: Information Technology and an Integrated Sustainability Framework." The Journal of Strategic Information Systems 20 (1): 63-79.

De Vasconcellos, E. S., J. A. Sousa, and D. C. Hambrick. 1989. "Key Success Factors: Test of a General Theory in the Mature Industrial-Product Sector." Strategic Management Journal 10 (4): 367-382.

Deephouse, D. L., and P. P. Heugens. 2009. "Linking Social Issues to Organizational Impact: The Role of Infomediaries and the Infomediary Process." Journal of Business Ethics 86 (4): 541-553.

Deng, J. L. 1982. Grey System Fundamental Method. Huazhong University of Science and Technology, Wuhan, China.

Diabat, A., D. Kannan, and K. Mathiyazhagan. 2014. "Analysis of Enablers for Implementation of Sustainable Supply Chain Management-A Textile Case.” Journal of Cleaner Production 83: 391-403.

Dinter, B. 2013. "Success Factors for Information Logistics Strategy—an Empirical Investigation." Decision Support Systems 54 (3): 1207-1218.

Dües, C. M., K. H. Tan, and M. Lim. 2013. "Green as the New Lean: How to Use Lean Practices as a Catalyst to Greening Your Supply Chain." Journal of Cleaner Production 40: 93-100.

Eisenhardt, K. M. 1989. "Building Theories from Case Study Research.” Academy of Management Review 14 (4): $532-550$.

Eltayeb, T. K., S. Zailani, and T. Ramayah. 2011. "Green Supply Chain Initiatives among Certified Companies in Malaysia and Environmental Sustainability: Investigating the Outcomes." Resources, Conservation and Recycling 55 (5): 495-506.

Fabbe-Costes, N., C. Roussat, M. Taylor and A. Taylor. 2014. "Sustainable Supply Chains: A Framework for Environmental Scanning Practices.” International Journal of Operations \& Production Management 34 (5): 664 694.

Fahimnia, B., J. Sarkis, and H. Davarzani. 2015. "Green Supply Chain Management: A Review and Bibliometrics Analysis." International Journal of Production Economics 162: 101-114.

Faisal, M. N. 2010. "Sustainable Supply Chains: A Study of Interaction among the Enablers." Business Process 
Management Journal 16 (3): 508-529.

Garetti, M. and Taisch, M. 2012. Sustainable manufacturing: trends and research challenges. Production Planning \& Control, 23(2-3): 83-104.

Gandhi, S., S. K. Mangla, P. Kumar, and Kumar, D. 2015. "Evaluating Factors in Implementation of Successful Green Supply Chain Management using DEMATEL: a Case Study. " International Strategic Management Review, 3 (12): 96-109.

Gandhi, S., S. K. Mangla, P. Kumar, and Kumar, D. 2016. "A combined Approach using AHP and DEMATEL for Evaluating Success Factors in Implementation of Green Supply Chain Management in Indian Manufacturing Industries. " International Journal of Logistics Research and Applications, 19 (6): 537-561.

Giunipero, L. C., R. E. Hooker, and D. Denslow. 2012. "Purchasing and Supply Management Sustainability: Drivers and Barriers." Journal of Purchasing and Supply Management 18 (4): 258-269.

Gölcük, İ., and A. Baykasoğlu. 2016. “An Analysis of DEMATEL Approaches for Criteria Interaction Handling within ANP." Expert Systems with Applications 46: 346-366.

Gold, S., R. Hahn, and S. Seuring. 2013. "Sustainable Supply Chain Management in "Base of the Pyramid" Food Projects- A Path to Triple Bottom Line Approaches for Multinationals?." International Business Review 22 (5): 784-799.

Golicic, S. L., and C. D. Smith. 2013. “A Meta-Analysis of Environmentally Sustainable Supply Chain Management Practices and Firm Performance.” Journal of Supply Chain Management 49 (2): 78-95.

Gopal, P. R. C., and J. Thakkar. 2016a. “Analysing Critical Success Factors to Implement Sustainable Supply Chain Practices in Indian Automobile Industry: A Case Study.” Production Planning \& Control 27 (12): $1005-1018$.

Gopal, P. R. C., and J. Thakkar. 2016b. "Sustainable Supply Chain Practices: An Empirical Investigation on Indian Automobile Industry." Production Planning \& Control 27 (1): 49-64.

Gopalakrishnan, K., Y. Y. Yusuf, A. Musa, T. Abubakar, and H. M. Ambursa. 2012. "Sustainable Supply Chain Management: A Case Study of British Aerospace (BAe) Systems." International Journal of Production Economics 140 (1): 193-203.

Govindan, K., R. Khodaverdi, and A. Jafarian. 2013. “A Fuzzy Multi Criteria Approach for Measuring Sustainability Performance of a Supplier Based on Triple Bottom Line Approach." Journal of Cleaner Production 47: $345-354$.

Govindan, K., R. Khodaverdi, and A. Vafadarnikjoo. 2015. "Intuitionistic Fuzzy based DEMATEL Method for Developing Green Practices and Performances in a Green Supply Chain.” Expert Systems with Applications 42 (20): 7207-7220.

Grimm, J. H., J. S. Hofstetter, and J. Sarkis. 2014. “Critical Factors for Sub-Supplier Management: A Sustainable Food Supply Chains Perspective.” International Journal of Production Economics 152: 159-173.

Gualandris, J., and M. Kalchschmidt. 2014. "Customer Pressure and Innovativeness: Their Role in Sustainable Supply Chain Management." Journal of Purchasing and Supply Management 20 (2): 92-103.

Gunasekaran, A., and A. Spalanzani. 2012. "Sustainability of Manufacturing and Services: Investigations for Research and Applications." International Journal of Production Economics 140 (1): 35-47.

Gupta, S., and O. D. Palsule-Desai. 2011. "Sustainable Supply Chain Management: Review and Research 
Opportunities." IIMB Management Review 23 (4): 234-245.

Haleem, A., Sushil, M. A. Qadri, and S. Kumar. 2012. "Analysis of Critical Success Factors of World-Class Manufacturing Practices: An Application of Interpretative Structural Modeling and Interpretative Ranking Process." Production Planning \& Control 23 (10-11): 722-734.

Hassini, E., C. Surti, and C. Searcy. 2012. "A Literature Review and A Case Study of Sustainable Supply Chains with a Focus on Metrics." International Journal of Production Economics 140 (1): 69-82.

Hsu, C. C., K. C. Tan, and S. H. Mohamad Zailani. 2016. "Strategic Orientations, Sustainable Supply Chain Initiatives, and Reverse Logistics: Empirical Evidence from an Emerging Market." International Journal of Operations \& Production Management 36 (1): 86-110.

Hsu, C. W., T. C. Kuo, S. H. Chen, and A. H. Hu. 2013. "Using DEMATEL to Develop A Carbon Management Model of Supplier Selection in Green Supply Chain Management." Journal of Cleaner Production 56: 164-172. India in Business (2016). A report on auto industry-July, 2016. Economic Diplomacy Division, Ministry of External Affairs, Government of India. Online available at: http://indiainbusiness.nic.in/newdesign/index.php? param=industry services landing $/ 329 / 1$

Irani, Z., M. M. Kamal, A. Sharif, and P. E. Love. 2017. "Enabling Sustainable Energy Futures: Factors Influencing Green Supply Chain Collaboration.” Production Planning \& Control 28 (6-8): 684-705.

Jabbour, A. B. L., and C. J. Jabbour. 2009. “Are Supplier Selection Criteria Going Green? Case Studies of Companies in Brazil." Industrial Management \& Data Systems 109 (4): 477-495.

Jabbour, C. J. C., and F. C. A. Santos. 2008. "Relationships between Human Resource Dimensions and Environmental Management in Companies: Proposal of a Model." Journal of Cleaner Production 16 (1): 51-58.

Kayden, J. S. 1991. "Market-Based Regulatory Approaches: A Comparative Discussion of Environmental and Land Use Techniques in the United States." Boston College Environmental Law Review 19 (3): 565-580.

Khavul, S., and G. D. Bruton. 2013. "Harnessing Innovation for Change: Sustainability and Poverty in Developing Countries." Journal of Management Studies 50 (2): 285-306.

Khompatraporn, C., and T. Somboonwiwat. 2017. "Causal Factor Relations of Supply chain Competitiveness Via Fuzzy DEMATEL Method for Thai Automotive Industry.” Production Planning \& Control 28 (6-8): 538-551.

Klassen, R. D. 2001. "Plant-Level Environmental Management Orientation: The Influence of Management Views and Plant Characteristics." Production and Operations Management 10 (3): 257-275.

Klassen, R. D., and A. Vereecke. 2012. "Social Issues in Supply Chains: Capabilities Link Responsibility, Risk (Opportunity), and Performance.” International Journal of Production Economics 140 (1): 103-115.

Kumar, D., and Z. Rahman. 2016. "Buyer Supplier Relationship and Supply Chain Sustainability: Empirical Study of Indian Automobile Industry." Journal of Cleaner Production 131: 836-848.

Kumar, S., S. Luthra, and A. Haleem. 2014. "Critical Success Factors of Customer Involvement in Greening the Supply Chain: An Empirical Study.” International Journal of Logistics Systems and Management 19 (3): $283-$ 310.

Kumar, S., S. Luthra, K. Govindan, N. Kumar and A. Haleem. 2016. "Barriers in Green Lean Six Sigma Product Development Process: An ISM Approach.” Production Planning \& Control 27 (7-8): 604-620. 
Lii, P. and F. I. Kuo. 2016. "Innovation-Oriented Supply Chain Integration for Combined Competitiveness and Firm Performance." International Journal of Production Economics 174: 142-155.

Lin, C., C. N. Madu, C. H. Kuei, H. L. Tsai, and K. N. Wang. 2015. "Developing an Assessment Framework for Managing Sustainability Programs: A Analytic Network Process Approach." Expert Systems with Applications 42 (5): 2488-2501.

Liu, S., Y. Lin, and J. Y. L. Forrest. 2010. Grey Systems: Theory and Applications. Springer Science \& Business Media, 68.

Luthra, S., D. Garg, and A. Haleem. 2015. "Critical Success Factors of Green Supply Chain Management for Achieving Sustainability in Indian Automobile Industry." Production Planning \& Control 26 (5): 339-362.

Mangla, S. K., J. Madaan, and F. T. Chan. 2013. "Analysis of Flexible Decision Strategies for Sustainability-Focused Green Product Recovery System.” International Journal of Production Research 51 (11): 3428-3442.

Mangla, S. K., P. Kumar, and M. K. Barua, 2015. "Risk Analysis in Green Supply Chain using Fuzzy AHP Approach: a Case Study. "Resources, Conservation and Recycling, 104: 375-390.

Mangla, S. K., K. Govindan, and S. Luthra. 2016. "Critical Success Factors for Reverse Logistics in Indian Industries: A Structural Model." Journal of Cleaner Production 129: 608-621.

Mani, V., A. Gunasekaran, T. Papadopoulos, B. Hazen, and R. Dubey. 2016. "Supply Chain Social Sustainability for Developing Nations: Evidence from India." Resources, Conservation and Recycling 111: 42-52.

Marshall, D., L. McCarthy, C. Heavey and P. McGrath. 2015. "Environmental and Social Supply Chain Management Sustainability Practices: Construct Development and Measurement." Production Planning \& Control 26 (8): 673690.

Miller, G., Pawloski, J. and Standridge, C. (2010) A case study of lean, sustainable manufacturing. Journal of Industrial Engineering and Management, 3(1), 11-32.

Min, H., and W. P. Galle. 2001. "Green Purchasing Practices of US Firms." International Journal of Operations \& Production Management 21 (9): 1222-1238.

Mitra, S., and P. P. Datta. 2014. "Adoption of Green Supply Chain Management Practices and Their Impact on Performance: An Exploratory Study of Indian Manufacturing Firms." International Journal of Production Research 52 (7): 2085-2107.

Muduli, K., K. Govindan, A. Barve, and Y. Geng. 2013. "Barriers to Green Supply Chain Management in Indian Mining Industries: A Graph Theoretic Approach." Journal of Cleaner Production 47: 335-344.

Mzembe, A. N., A. Lindgreen, F. Maon, and J. Vanhamme. 2016. "Investigating the Drivers of Corporate Social Responsibility in the Global Tea Supply Chain: A Case Study of Eastern Produce Limited in Malawi." Corporate Social Responsibility and Environmental Management (In Press), DOI: 10.1002/csr.1370 (accessed on: January 10, 2016).

Ogunbiyi, O., A. Oladapo, and J. Goulding. 2014. "An Empirical Study of the Impact of Lean Construction Techniques on Sustainable Construction in the UK." Construction Innovation 14 (1): 88-107.

Piercy, N. and N. Rich. 2015. "The Relationship Between Lean Operations and Sustainable Operations.” International Journal of Operations \& Production Management 35 (2): 282-315. 
Prakash, C., and M. K. Barua. 2015. "Integration of AHP-TOPSIS Method for Prioritizing the Solutions of Reverse Logistics Adoption to Overcome its Barriers under Fuzzy Environment." Journal of Manufacturing Systems 37 (3): 599-615.

Rahman, S., and N. Subramanian. 2012. "Factors for Implementing End-Of-Life Computer Recycling Operations in Reverse Supply Chains." International Journal of Production Economics 140 (1): 239-248.

Rajesh, R., V. Ravi and R. Venkata Rao. 2015. "Selection of Risk Mitigation Strategy in Electronic Supply Chains using Grey Theory and Digraph-Matrix Approaches." International Journal of Production Research 53 (1): 238257.

Ramanathan, U., Y. Bentley, and G. Pang. 2014. "The Role of Collaboration in the UK Green Supply Chains: An Exploratory Study of the Perspectives of Suppliers, Logistics and Retailers." Journal of Cleaner Production 70: 231-241.

Sajjad, A., G. Eweje, and D. Tappin. 2015. "Sustainable Supply Chain Management: Motivators and Barriers." Business Strategy and the Environment 24 (7): 643-655.

Sarkis, J., P. Gonzalez-Torre, and B. Adenso-Diaz. 2010. "Stakeholder Pressure and the Adoption of Environmental Practices: The Mediating Effect of Training." Journal of Operations Management 28(2): 163-176.

Sarkis, J. 2012. "Models for Compassionate Operations." International Journal of Production Economics 139(2): 359365 .

Seghezzo, L. 2009. “The Five Dimensions of Sustainability.” Environmental Politics 18 (4): 539-556.

Seker, S., F. Recal, and H. Basligil. 2017. "A Combined DEMATEL and Grey System Theory Approach for Analysing Occupational Risks: A Case Study in Turkish Shipbuilding Industry." Human and Ecological Risk Assessment: An International Journal , DOI:10.1080/10807039.2017.1308815

Seleem, S. N., E. A. Attia, and A. El-Assal. 2016. "Managing Performance Improvement Initiatives Using DEMATEL Method with Application Case Study.” Production Planning \& Control 27 (7-8): 637-649.

Seuring, S. 2013. "A Review of Modelling Approaches for Sustainable Supply Chain Management." Decision Support Systems 54 (4): 1513-1520.

Seuring, S., and M. Müller. 2008. "From a Literature Review to a Conceptual Framework for Sustainable Supply Chain Management." Journal of Cleaner Production 16 (15): 1699-1710.

Silvestre, B. 2015. "Sustainable Supply Chain Management in Emerging Economies: Environmental Turbulence, Institutional Voids and Sustainability Trajectories." International Journal of Production Economics 167: 156169.

Soosay, C. A., P. W. Hyland, and M. Ferrer. 2008. "Supply Chain Collaboration: Capabilities for Continuous Innovation." Supply Chain Management: An International Journal 13 (2): 160-169.

Su, C. M., D. J. Horng, M. L. Tseng, A. S. Chiu, K. J. Wu, and H. P. Chen. 2016. "Improving Sustainable Supply Chain Management Using A Novel Hierarchical Grey-DEMATEL Approach." Journal of Cleaner Production 134: 469-481.

Subramanian, N., and A. Gunasekaran. 2015. "Cleaner Supply-Chain Management Practices for Twenty-First-Century Organizational Competitiveness: Practice-Performance Framework and Research Propositions." International 
Journal of Production Economics 164: 216-233.

Subramanian, N., A. Gunasekaran, M. Abdulrahman, and C. Liu. 2014. "Factors for Implementing End-of-Life Product Reverse Logistics in the Chinese Manufacturing Sector." International Journal of Sustainable Development \& World Ecology 21 (3) : 235-245.

Taticchi, P., P. Garengo, S. S. Nudurupati, F. Tonelli, and R. Pasqualino. 2015. "A Review of Decision-Support Tools and Performance Measurement and Sustainable Supply Chain Management." International Journal of Production Research 53 (21): 6473-6494.

Tseng, M. L., R. R. Tan, and A. B. Siriban-Manalang. 2013. "Sustainable Consumption and Production for Asia: Sustainability through Green design and Practice." Journal of Cleaner Production 40: 1-5.

Tseng, M., M. Lim, and W. P. Wong. 2015. "Sustainable Supply Chain Management: A Closed-Loop Network Hierarchical Approach." Industrial Management \& Data Systems 115 (3): 436-461.

Tseng, S. C., and S. W. Hung. 2014. "A Strategic Decision-Making Model Considering the Social Costs of Carbon Dioxide Emissions for Sustainable Supply Chain Management." Journal of Environmental Management 133: $315-322$.

Turker, D., and C. Altuntas. 2014.” Sustainable Supply Chain Management in the Fast Fashion Industry: An Analysis of Corporate Reports." European Management Journal 32 (5): 837-849.

Vachon, S., and R. D. Klassen. 2008. "Environmental Management and Manufacturing Performance: The Role of Collaboration in the Supply Chain.” International Journal of Production Economics 111 (2): 299-315.

Walker, H., and N. Jones. 2012. "Sustainable Supply Chain Management across the UK Private Sector." Supply Chain Management: An International Journal 17 (1): 15-28.

Walker, H., L. Di Sisto, and D. Mc Bain. 2008. "Drivers and Barriers to Environmental Supply Chain Management Practices: Lessons from the Public and Private Sectors." Journal of purchasing and supply management 14 (1): 69-85.

Wilhelm, M. M., C. Blome, V. Bhakoo, and A. Paulraj. 2016. "Sustainability in Multi-Tier Supply Chains: Understanding the Double Agency Role of the First-Tier Supplier." Journal of Operations Management 41: 4260.

Wittstruck, D., and F. Teuteberg. 2012. "Understanding the Success Factors of Sustainable Supply Chain Management: Empirical Evidence from the Electrics and Electronics Industry." Corporate Social Responsibility and Environmental Management 19 (3): 141-158.

Xia, X., K. Govindan, and Q. Zhu. 2015. "Analysing Internal Barriers for Automotive Parts Remanufacturers in China using Grey-DEMATEL Approach.” Journal of Cleaner Production 87: 811-825.

Yin, R. K. 2013. Case Study Research: Design and Methods. Sage Publications, Oaks.

Zailani, S., K. Jeyaraman, G. Vengadasan, and R. Premkumar. 2012. "Sustainable Supply Chain Management (SSCM) in Malaysia: A Survey." International Journal of Production Economics 140 (1): 330-340.

Zhang, M., K. S. Pawar, and S. Bhardwaj. 2017. "Improving Supply Chain Social Responsibility through Supplier Development." Production Planning \& Control: 1-12.

Zhu, Q., J. Sarkis, and K. H. Lai. 2007. “Green Supply Chain Management: Pressures, Practices and Performance 
Within the Chinese Automobile Industry." Journal of Cleaner Production 15 (11): 1041-1052.

Zhu, Q., J. Sarkis, and K. H. Lai. 2013. "Institutional-Based Antecedents and Performance Outcomes of Internal and External Green Supply Chain Management Practices." Journal of Purchasing and Supply Management 19 (2): $106-117$.

\section{$\underline{\text { Appendices }}$}

\section{Appendix -A}

The crisp relationship matrix (B) was computed through average grey matrix. The grey numbers are converted to crisp numbers by the modified-CFCS (Converting Fuzzy data into Crisp Scores) method (Xia et al. 2015) involving a three-step procedure described as follows.

(i) Lower and upper normalised values.

$$
\underline{\otimes} \dot{A}_{x y}=\left(\underline{\otimes} \check{A}_{x y}-{ }^{\min } \underline{\otimes} \check{A}_{x y}\right) / \Delta_{\min }^{\max }
$$

Where $\underline{\otimes} \dot{A}_{x y}$ represents the normalised lower limit value of the grey number $\underline{\otimes} \check{A}_{x y}$

$$
\bar{\otimes} \dot{A}_{x y}=\left(\bar{\otimes} \check{A}_{x y}-{ }_{y}^{\min } \bar{\otimes} \check{A}_{x y}\right) / \Delta_{\min }^{\max }
$$

Where $\bar{\otimes} \dot{A}_{x y}$ represents the normalised upper limit value of the grey number $\bar{\otimes} \check{A}_{x y}$

$$
\begin{aligned}
& \Delta_{\min }^{\max }={ }_{y}^{\max } \bar{\otimes} \check{A}_{x y}-{ }_{y}^{\min } \underline{\otimes} \check{A}_{x y} \\
& \text { (ii) } \quad \text { Calculate total normalised crisp value } \\
& B_{x y}=\left(\frac{\left(\underline{\otimes} \dot{A}_{x y}\left(1-\underline{\otimes} \dot{A}_{x y}\right)+\left(\bar{\otimes} \dot{A}_{x y} \times \bar{\otimes} \dot{A}_{x y}\right)\right.}{\left(1-\underline{\otimes} \dot{A}_{x y}+\bar{\otimes} \dot{A}_{x y}\right)}\right) \\
& \text { (iii) } \quad \text { Compute final crisp values } \\
& B_{x y}^{*}=\left(\min \underline{\otimes} \dot{A}_{x y}+\left(B_{x y} \times \Delta_{\text {min }}^{\text {max }}\right)\right)
\end{aligned}
$$

Where,

$$
B=\left[B_{x y}^{*}\right]
$$




\section{Appendix-B}

Name of the organisation......................................, India

\section{Sustainability Initiatives in Supply Chains in Indian Context}

Dear Ir./Professor/Assoc. Prof./Dr./Mr./Mrs./Ms.,

Thank you for participating in this study.

This study provides an opportunity for you to participate and share your opinions in the development of a framework on 'Sustainability Initiatives in Supply Chains in Indian Context'. The present paper uncovers the Critical Success Factors (CSFs) for effective adoption of sustainability initiatives in the supply chain in Indian context. The outcome of this survey is aimed at i) Understanding and uncovering the most common CSFs to the effective implementation of supply chain sustainability; ii) Analysing the identified sustainability oriented CSFs by dividing them into cause and effect groups to understand their causal relations.

We are keen to receive feedback and learn from your experiences.

Please note that all responses are confidential. No individuals will be named as a result of the survey. You will not be contacted as a result of your responses to this survey. Your invaluable response will be used for academic research purposes only.

Thank you for your kind response.

Regards, 


\section{SURVEY QUESTIONNAIRE}

This questionnaire consists of three sections. Section A deals with the general information of the respondents and their respective background where they work. Section B helps in selecting the most suitable CSFs and exploring their significance to achieving supply chain sustainability. Section $\mathrm{C}$ assists in examining the causal relations of the selected CSFs.

\section{SECTION A: General information}

Please hightight only one choice in each question as follows:

1. What is your professional qualification level?

(a) Graduate

(b) Post Graduate

(c) Doctorate

(d) If any other, please specify.

2. What is your work experience?

(a) Less than 5 Years

(b) 5 to 10 Years

(c) 11 to 15 Years

(d) 16 to 20 Years

(e) Greater than 20 Years

\section{What is size of your organisation?}

(a) Less than 50 Employees

(b) 51 to 250 Employees

(c) 251 - 500 Employees

(d) $501-1000$ employees

(e) $1001-5000$ employees

(f) Greater than 5001 employees

4. How will you classify your sector and work profile?

(a) Private Sector - please specify nature of your work

(b) Public Sector - please specify nature of your work

(c) Multinational Corporation - please specify nature of your work

(d) Regulatory Bodies - please specify nature of your work

(e) Mixed public and private ownership - please specify nature of your work

(f) If any other, please specify........... 
SECTION B: Selecting the most common CSFs in effective adoption of sustainability initiatives in a supply chain context

We selected sixteen CSFs in effective adoption of sustainability initiatives in supply chains as provided in the response sheet based on related literature. However, there may be several other types of CSFs in accomplishing supply chain sustainability efficiently. Thus, we aim to list the most common CSFs through your (experts) response. Please rate the following barriers on 7 point Likert scale (where, 1-least relevant and 7-most relevant). Further, you are also free to add/delete any other factor which you think is significant to the point of supply chain sustainability in Indian context and should be included into the list. Please note that numbering mentioned with the factors (CSF1, 2, $3 . . ., 16)$ does not their indicate level of importance.

CSFs in effective adoption of sustainability initiatives in a supply chain context as reported in the literature

\begin{tabular}{|l|l|}
\hline $\begin{array}{l}\text { CSFs in effective adoption of sustainability initiatives in a } \\
\text { supply chain context }\end{array}$ & $\begin{array}{l}\text { Please rate your response (using 7 point } \\
\text { Likert scale (where, 1-least relevant and 7-most } \\
\text { relevant) }\end{array}$ \\
\hline Government legalisation (CSF1) & \\
\hline Top management support (CSF2) & \\
\hline $\begin{array}{l}\text { Ecological considerations in organisations' policies and } \\
\text { missions (CSF3) }\end{array}$ & \\
\hline Societal considerations (CSF4) & \\
\hline Supply chain members' collaborations (CSF5) & \\
\hline Technology development and process innovation (CSF6) & \\
\hline Communication and information technology (CSF7) & \\
\hline Training (CSF8) & \\
\hline Green design and purchasing (CSF9) & \\
\hline Reverse logistics and waste minimisation (CSF10) & \\
\hline Ethical and safe practices (CSF11) & \\
\hline Customer involvement and encouragement (CSF12) & \\
\hline Community welfare and development (CSF13) & \\
\hline Economic considerations (CSF14) & \\
\hline Competitiveness and brand image considerations (CSF15) & \\
\hline Investment recovery (CSF16) & \\
\hline Please add/modify for the relevant CSF (in your opinion) & \\
\hline Please add/modify for the relevant CSF (in your opinion) & \\
\hline
\end{tabular}




\section{SECTION C: Analysing the identified sustainability oriented CSFs to understand their causal relations}

After finalising the most common CSFs to supply chain sustainability, it is needed to analyse them to understand their causal relations. Therefore, it needs to construct the direct relation matrix for the identified factors. In view of that, please put your response in the direct relation matrix for the selected CSFs. Please use the given linguistic assessment and associated Grey scales for entering your responses.

\section{Linguistics assessment and associated Grey scales}

\begin{tabular}{l|c|c}
\hline Linguistics assessment & Assigned Grey numbers & Crisp values \\
\hline No influence (N) & $(0,0.1)$ & 0 \\
\hline Very low influence (VL) & $(0.1,0.3)$ & 1 \\
\hline Low influence (L) & $(0.2,0.5)$ & 2 \\
\hline Medium influence (M) & $(0.4,0.7)$ & 3 \\
\hline High influence (H) & $(0.6,0.9)$ & 5 \\
\hline Very high influence (VH) & $(0.9,1.0)$ & \\
\hline
\end{tabular}

Name of Respondent:

Designation:

Organisation:

Email:

Date:

Place:

Thank you very much for completing this questionnaire

If you have any comments about this questionnaire or issues involved please write them in the box given below 


\section{$\underline{\text { Appendix-C }}$}

\section{Sensitivity Analysis Runs}

\section{Sensitivity analysis run 2}

Similarly, in sensitivity analysis run 2; when expert 2 has highest weightage (0.3) and other experts have equal weightage $(0.1)$ then the cause-effect diagram, Figure B1 indicates that CSF $1>$ CSF2 $>$ CSF6 are three most important causal factors and CSF $11>$ CSF14 $>$ CSF $15>$ CSF13 are the effect factors.

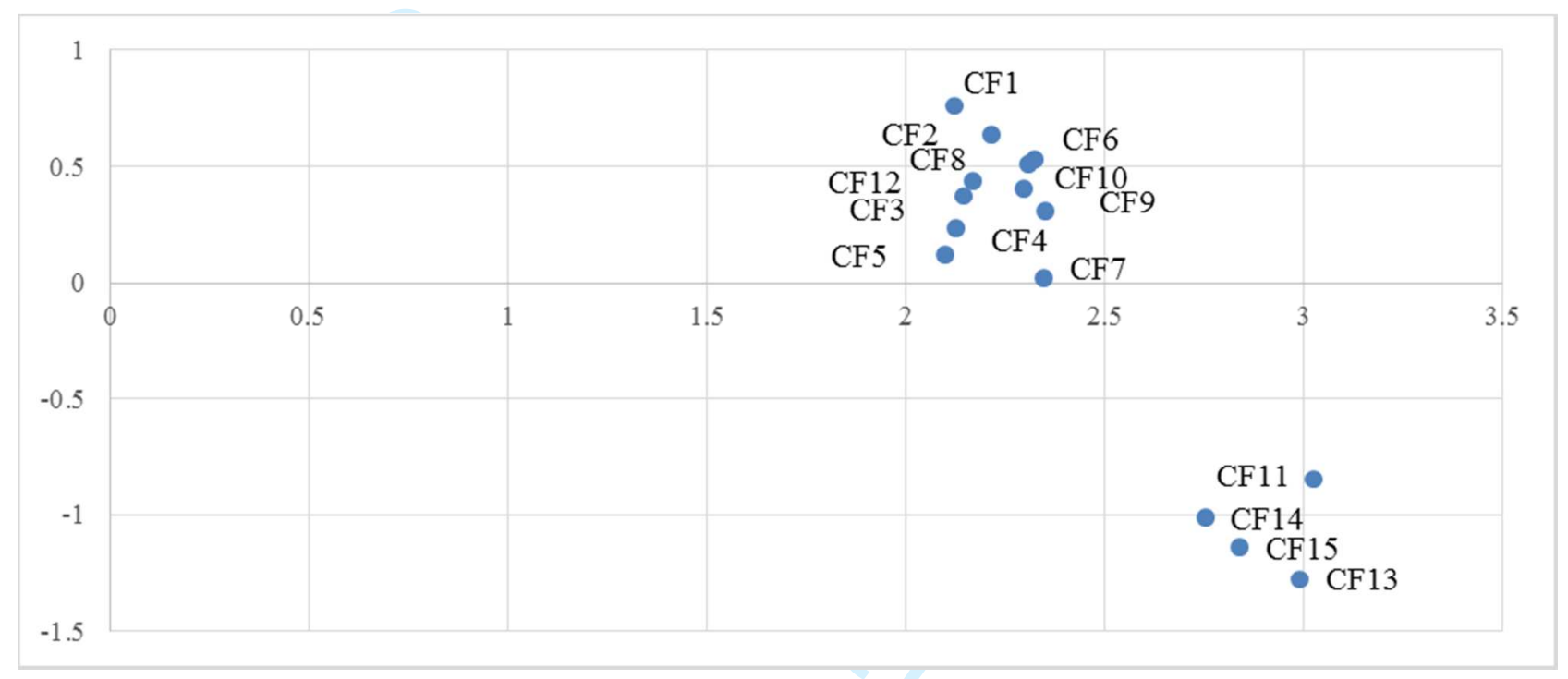

Figure B1: Causal relationship illustration among CSFs for effective adoption of sustainability initiatives in supply chain obtained from sensitivity analysis run 2

\section{Sensitivity analysis run 3}

In sensitivity analysis run 3, where expert 3 has assigned weightage (0.3) and other experts have identical weightage $(0.1)$, is found that CSF1>CSF2 $>$ CSF6 are three most important causal factors and CSF11 $>$ CSF14 $>$ CSF15 $>$ CSF13 are the effect factors (see Figure B2). 


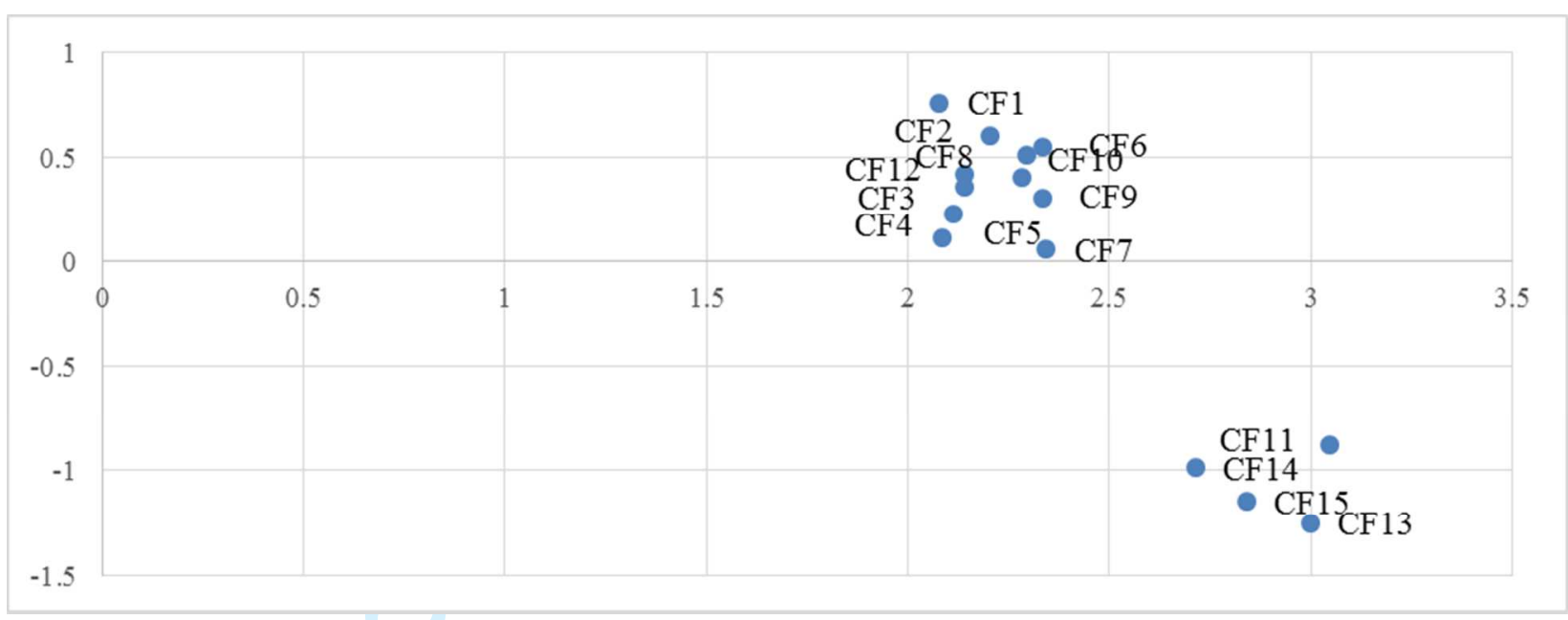

Figure B2: Causal relationship illustration among CSFs for effective adoption of sustainability initiatives in supply chain obtained from sensitivity analysis run 3

\section{Sensitivity analysis run 4}

In sensitivity run $4, \mathrm{CSF} 1>\mathrm{CSF} 2>\mathrm{CSF} 6$ are three most important causal factors and CSF11 $>$ CSF14 $>$ CSF15 $>$ CSF13 are the effect factors (see Figure B3).

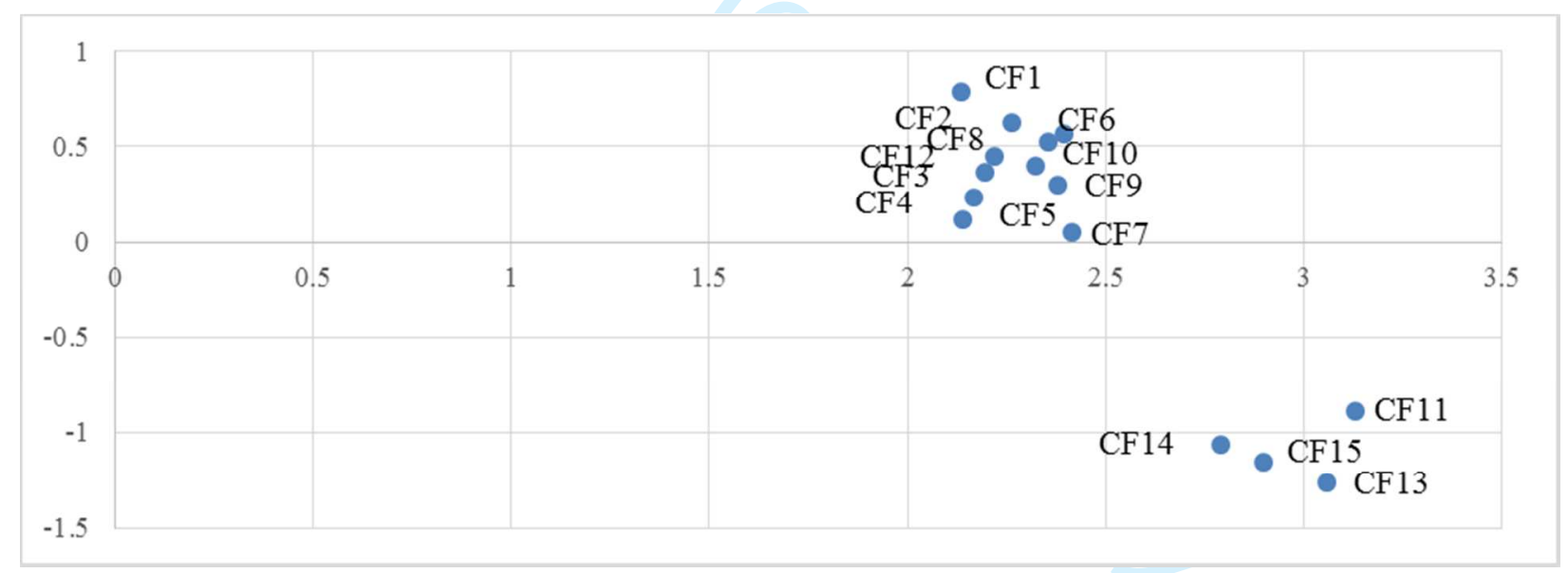

Figure B3: Causal relationship illustration among CSFs for effective adoption of sustainability initiatives in supply chain obtained from sensitivity analysis run 4

\section{Sensitivity analysis run 5}

In sensitivity run $5, \mathrm{CSF} 1>\mathrm{CSF} 2>\mathrm{CSF} 6$ are three most important causal factors and CSF11 $>$ CSF14 $>$ CSF15 $>$ CSF13 are the effect factors (see Figure B4). 


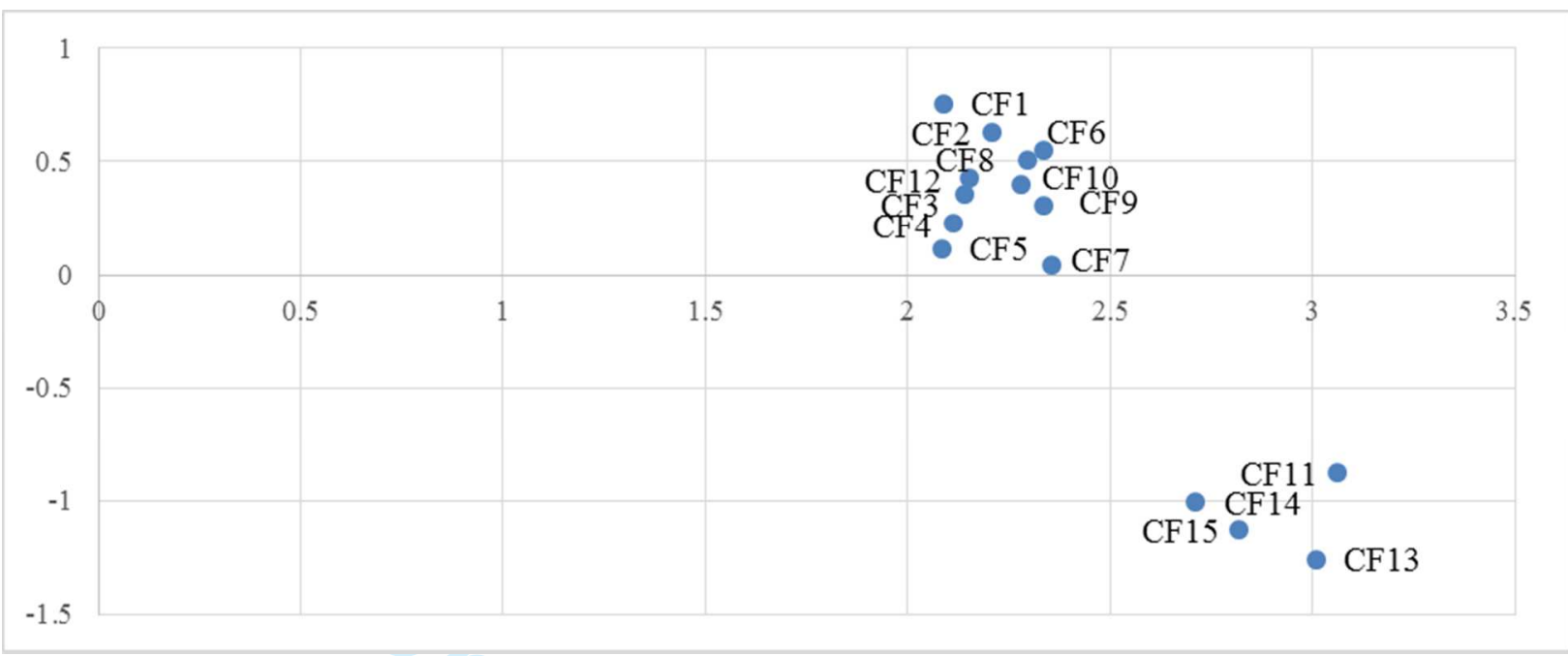

Figure B4: Causal relationship illustration among CSFs for effective adoption of sustainability initiatives in supply chain obtained from sensitivity analysis run 5

\section{Sensitivity analysis run 6}

In sensitivity run $6, \quad \mathrm{CSF} 1>\mathrm{CSF} 2>\mathrm{CSF} 6$ are three causal factors and CSF11 $>$ CSF14 $>$ CSF15 $>$ CSF13 are the effect factors (see Figure B5).

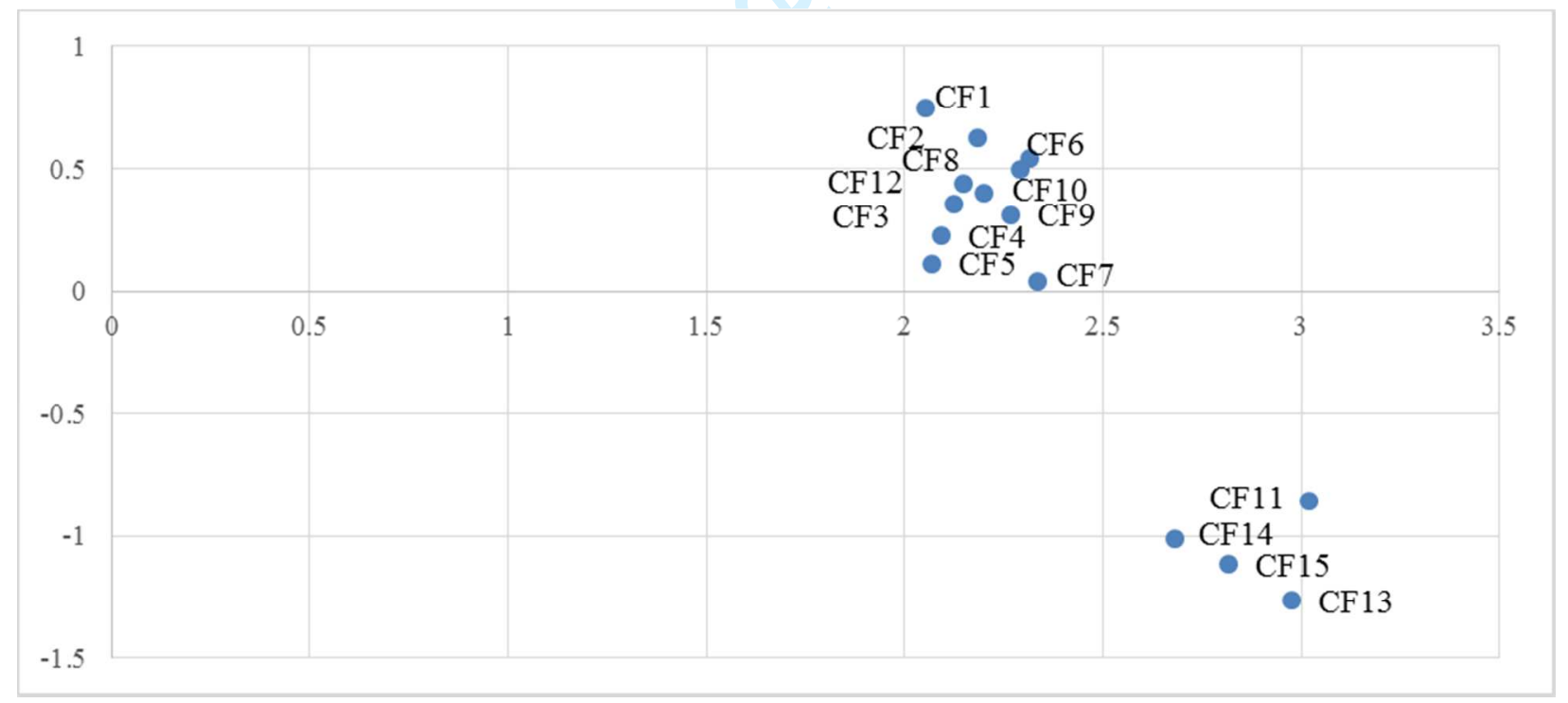

Figure B5: Causal relationship illustration CSFs for effective adoption of sustainability initiatives in supply chain obtained from sensitivity analysis run 6 


\section{Sensitivity analysis run 7}

In sensitivity run $7, \mathrm{CSF} 1>\mathrm{CSF} 5>\mathrm{CSF} 12>\mathrm{CSF} 4>\mathrm{CSF} 3$ are the five important factors, $\mathrm{CSF} 1>\mathrm{CSF} 2>\mathrm{CSF} 6$ are three causal factors and $\mathrm{CSF} 11>\mathrm{CSF} 14>\mathrm{CSF} 15>\mathrm{CSF} 13$ are the effect factors (see Figure B6).

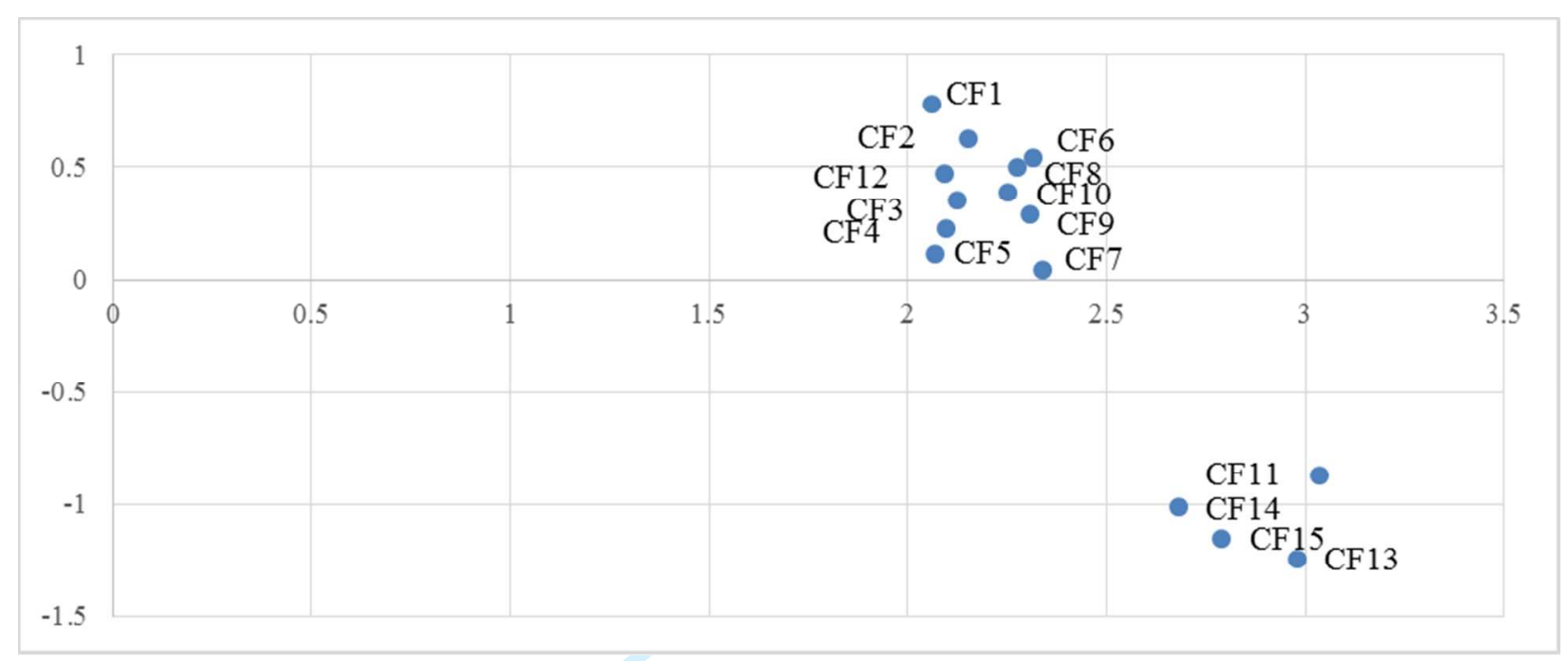

Figure B6: Causal relationship illustration among CSFs for effective adoption of sustainability initiatives in supply chain obtained from sensitivity analysis run 7

\section{Sensitivity analysis run 8}

Sensitivity run 8 shows that $\mathrm{CSF} 1>\mathrm{CSF} 2>\mathrm{CSF} 6$ are three most important causal factors and CSF11 $>$ CSF14 $>$ CSF15 $>$ CSF13 are the effect factors (see Figure B7).

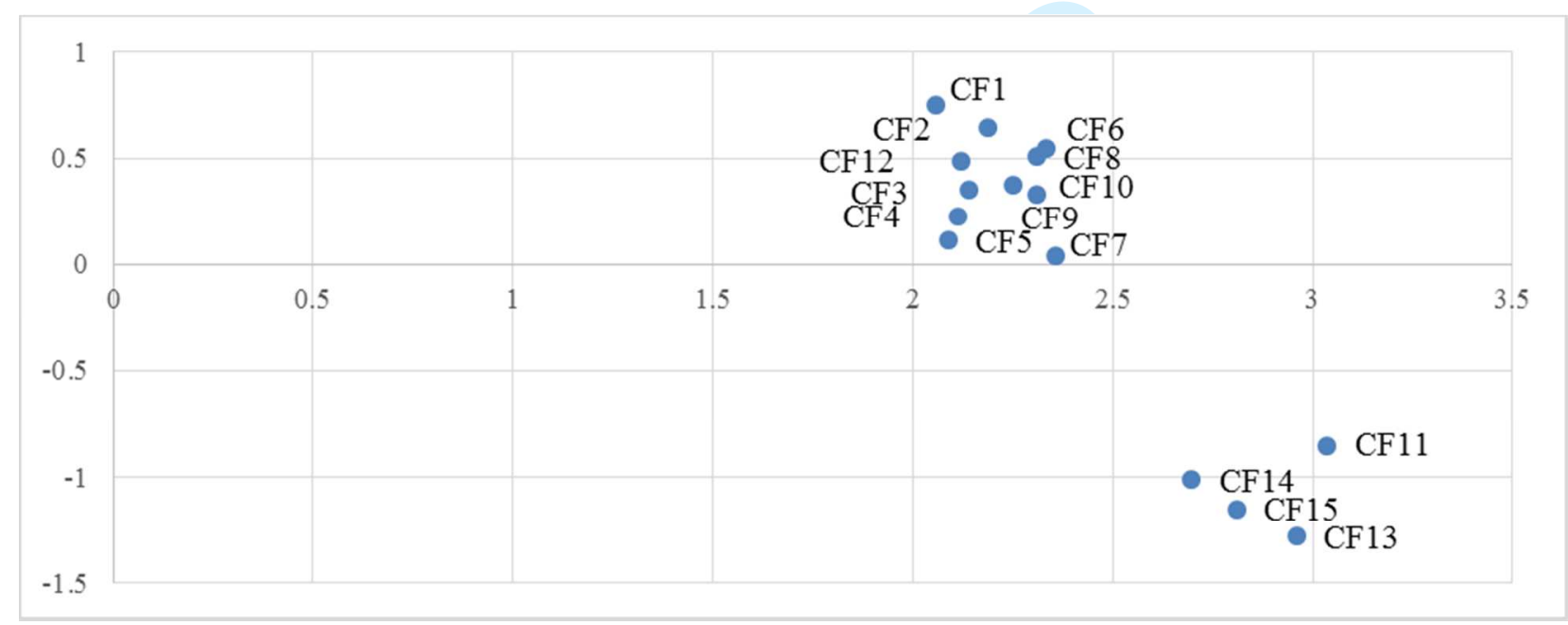

Figure B7: Causal relationship illustration among CSFs for effective adoption of sustainability initiatives in supply chain obtained from sensitivity analysis run 8 


\section{$\underline{\text { List of Figures }}$}

Figure 1: Proposed research framework

Figure 2: Diagraph represents causal relationship among critical success factors for effective adoption of sustainability initiatives in supply chain

Figure 3: Causal relationship illustration among critical success factors for effective adoption of sustainability initiatives in supply chain obtained from sensitivity analysis run 1

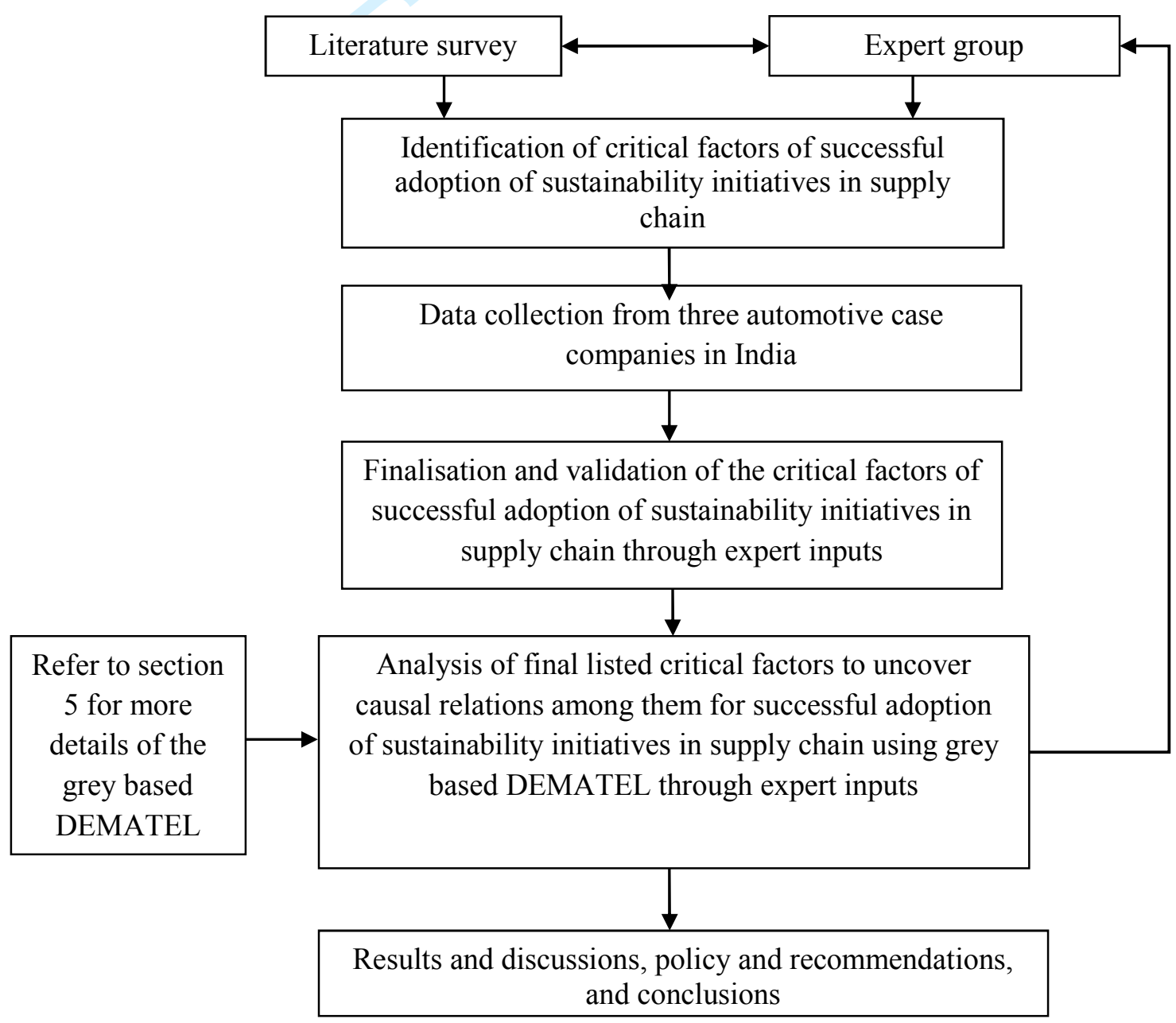

Figure 1: Proposed research framework 


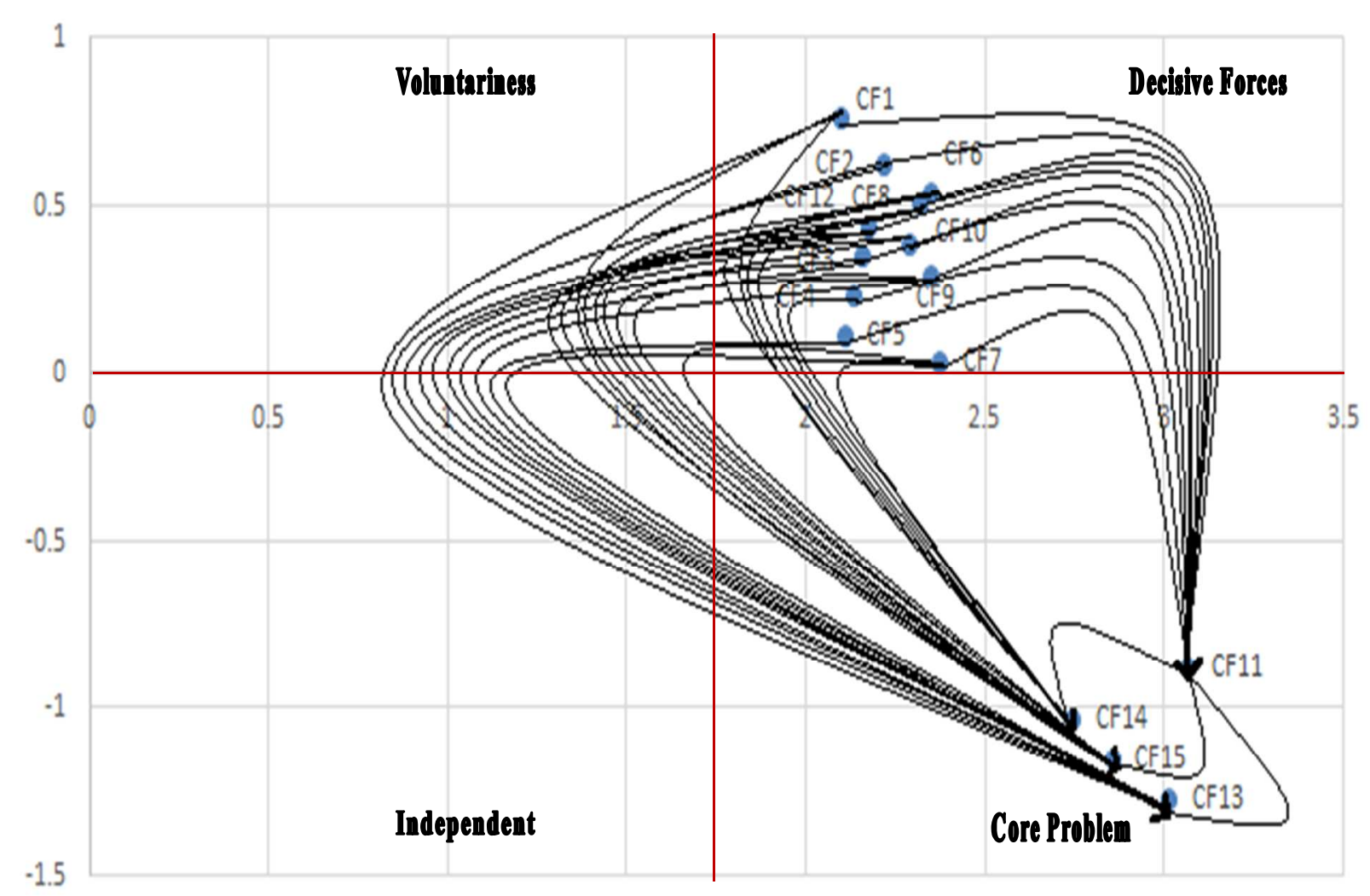

$\mathrm{X}$ axis- ( $\mathrm{R}+\mathrm{D})$; $\mathrm{Y}$ axis- $(\mathrm{R}-\mathrm{D})$

Figure 2: Diagraph represents causal relationship among critical success factors for effective adoption of sustainability initiatives in supply chain

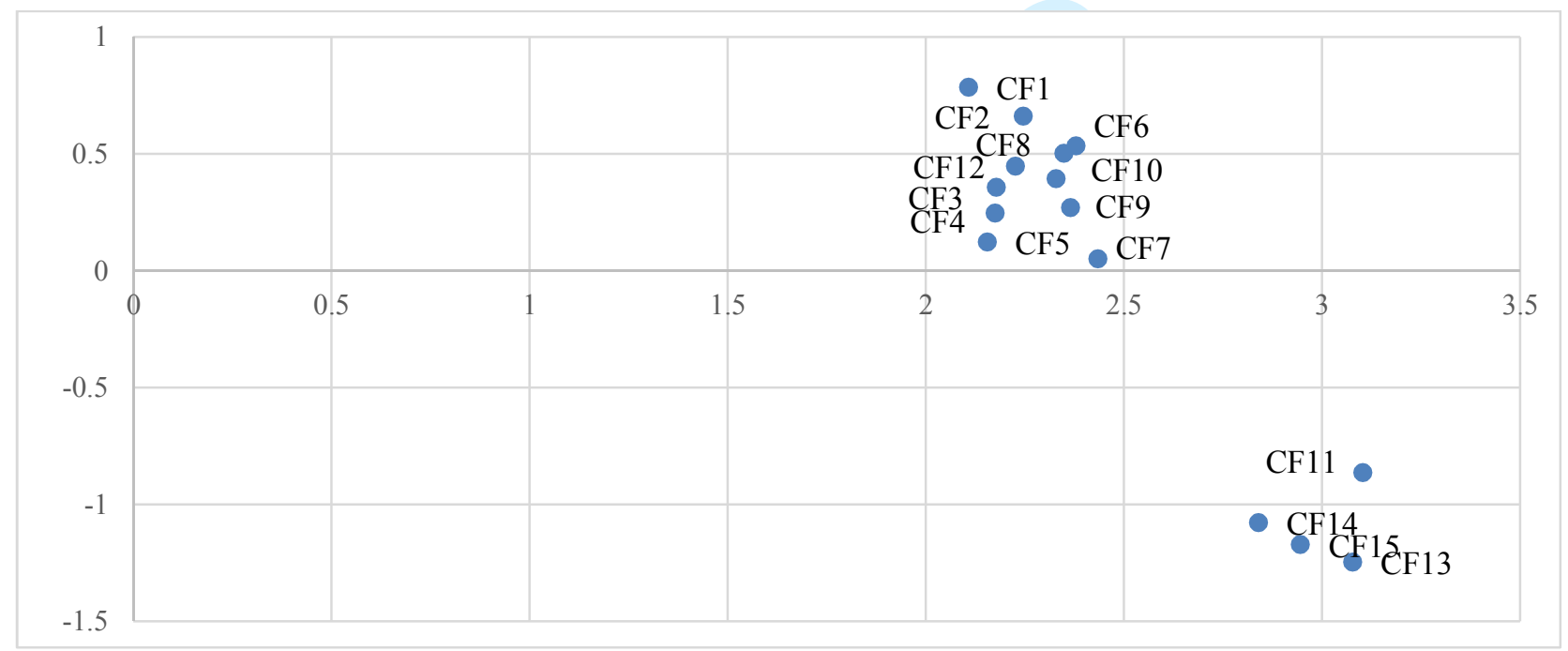

Figure 3: Causal relationship illustration among critical success factors for effective adoption of sustainability initiatives in supply chain obtained from sensitivity analysis run 1 


\section{List of Tables}

Table 1: Modelling techniques incorporating sustainability in supply chain

Table 2: Linguistics assessment and associated Grey scales

Table 3: Brief description of case companies

Table 4: Grey relationship matrix for critical success factors for effective adoption of sustainable initiatives in the automotive industry supply chain by Expert 1

Table 5: Average Grey relationship matrix for critical success factors for effective adoption of sustainability initiatives in the automotive industry supply chain

Table 6: Normalised crisp relationship matrix for critical success factors for effective adoption of sustainability initiatives in the automotive industry supply chain

Table 7: Final crisp relationship matrix for critical success factors for effective adoption of sustainability initiatives in the automotive industry supply chain

Table 8: Normalised direct relationship matrix for critical success factors for effective adoption of sustainability initiatives in the automotive industry supply chain

Table 9: Total relationship matrix for critical success factors for effective adoption of sustainability initiatives in the automotive industry supply chain

Table 10: Cause/effect parameters for critical success factors for effective adoption of sustainability initiatives in the automotive industry supply chain

Table 11: Weights assigned for eight experts during sensitivity analysis

Table 12: Sensitivity analysis of critical success factors for effective adoption of sustainability initiatives in the supply chains 
Table 1: Modelling techniques incorporating sustainability in supply chain

\begin{tabular}{|c|c|c|c|}
\hline $\begin{array}{l}\text { S. } \\
\text { No. }\end{array}$ & Researcher (Year) & Modelling techniques used & Issues addressed \\
\hline 1 & $\begin{array}{l}\text { Bai and Sarkis } \\
(2010)\end{array}$ & Grey theory and Rough set & $\begin{array}{l}\text { Sustainability focused supplier } \\
\text { selection }\end{array}$ \\
\hline 2 & Faisal (2010) & Interpretive Structural Modelling (ISM) & Enablers of SSCM \\
\hline 3 & $\begin{array}{l}\text { Büyüközkan and } \\
\text { Çifçi (2011) }\end{array}$ & Fuzzy Analytical Hierarchical Process (FAHP) & $\begin{array}{l}\text { Sustainability focused supplier } \\
\text { selection }\end{array}$ \\
\hline 4 & $\begin{array}{l}\text { Amindoust et al. } \\
(2012)\end{array}$ & Fuzzy inference system & $\begin{array}{l}\text { Sustainability focused supplier } \\
\text { selection }\end{array}$ \\
\hline 5 & $\begin{array}{l}\text { Al Zaabi et al. } \\
(2013)\end{array}$ & ISM & Barriers to implement SSCM \\
\hline 6 & $\begin{array}{l}\text { Govindan et } \\
\text { al.(2013) }\end{array}$ & $\begin{array}{l}\text { Fuzzy TOPSIS (The Technique for Order of } \\
\text { Preference by Similarity to Ideal Solution) }\end{array}$ & $\begin{array}{l}\text { Sustainable } \\
\text { performance measurement }\end{array}$ \\
\hline 7 & Mangla et al. (2013) & ISM & $\begin{array}{l}\text { Sustainability focused product } \\
\text { recovery systems }\end{array}$ \\
\hline 8 & $\begin{array}{l}\text { Bai and Sarkis } \\
(2014)\end{array}$ & Rough set theory and Data envelopment analysis & $\begin{array}{l}\text { Sustainable supplier } \\
\text { performance measurement }\end{array}$ \\
\hline 9 & Diabat et al. (2014) & 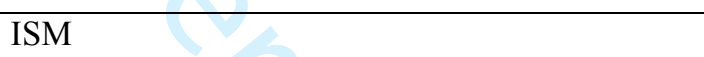 & Enablers of SSCM \\
\hline 10 & $\begin{array}{l}\text { Tseng and Hung } \\
\text { (2014) }\end{array}$ & Mixed integer programming & $\begin{array}{ll}\text { SSCM } & \text { performance } \\
\text { management } & \end{array}$ \\
\hline 11 & Azadi et al. (2015) & Fuzzy DEA (Data Envelopment Analysis) & $\begin{array}{l}\text { Sustainable supplier } \\
\text { performance evaluation }\end{array}$ \\
\hline 12 & Lin et al. (2015) & Analytical Network Process (ANP) & $\begin{array}{l}\text { Sustainability focused Supplier } \\
\text { selection }\end{array}$ \\
\hline 13 & Tseng et al. (2015) & Fuzzy Delphi Method (FDM) and ANP & $\begin{array}{l}\text { Sustainable supplier } \\
\text { performance measurement }\end{array}$ \\
\hline 14 & $\begin{array}{l}\text { Gopal and Thakkar } \\
\text { (2016a) }\end{array}$ & ISM & SSCM practices \\
\hline 15 & $\begin{array}{l}\text { Gopal and Thakkar } \\
\text { (2016b) }\end{array}$ & Structural Equation Modelling (SEM) & SSCM practices \\
\hline 16 & Su et al. (2016) & Grey based DEMATEL & Sustainability focused Supplier \\
\hline
\end{tabular}

Table 2: Linguistics assessment and associated Grey scales

\begin{tabular}{l|c|c}
\hline Linguistics assessment & Assigned Grey numbers & Crisp values \\
\hline No influence (N) & $(0,0.1)$ & 0 \\
\hline Very low influence (VL) & $(0.1,0.3)$ & 2 \\
\hline Low influence (L) & $(0.2,0.5)$ & 3 \\
\hline Medium influence (M) & $(0.4,0.7)$ & 4 \\
\hline High influence (H) & $(0.6,0.9)$ & 5 \\
\hline Very high influence (VH) & $(0.9,1.0)$ & \\
\hline
\end{tabular}


Table 3: Brief description of case companies

\begin{tabular}{|c|c|c|c|}
\hline $\begin{array}{l}\text { Business } \\
\text { Characteristics }\end{array}$ & Case Company 1 & Case Company 2 & Case Company 3 \\
\hline Turnover (in INR) & 150-160 Million & 140-150 Million & 120-130 Million \\
\hline Employees & More than 2000 & More than 2000 & $1500-2000$ \\
\hline $\begin{array}{l}\text { Year of } \\
\text { establishment }\end{array}$ & 1983 & 1984 & 1987 \\
\hline Certifications & OHSAS 18001 and ISO 14001 & $\begin{array}{l}\text { ISO 14001, ISO TS } \\
16949, \text { OHSAS } 18001\end{array}$ & $\begin{array}{l}\text { ISO } 9001, \text { ISO } 14001, \text { TS } \\
16949 \text { and OHSAS } 18001\end{array}$ \\
\hline $\begin{array}{l}\text { Products } \\
\text { manufactured type/ } \\
\text { Specialization }\end{array}$ & $\begin{array}{l}\text { Various automotive }(2,3,4 \text { and } \\
\text { commercial wheelers }) \\
\text { components }\end{array}$ & 2 wheeler components & $\begin{array}{l}2 \text { and } 4 \text { wheelers automotive } \\
\text { components }\end{array}$ \\
\hline Type of business & Manufacturer, supplier & Manufacturer, supplier & Manufacturer, supplier \\
\hline
\end{tabular}


Table 4: Grey relationship matrix for CSFs for effective adoption of sustainability initiatives in the automotive industry supply chain by Expert 1

\begin{tabular}{|c|c|c|c|c|c|c|c|c|c|c|c|c|c|c|c|}
\hline CSFs & CSF1 & CSF2 & CSF3 & CSF4 & CSF5 & SF6 & CSF7 & CSF8 & SSF9 & CSF10 & CSF11 & CSF12 & CSF13 & CSF14 & CSF15 \\
\hline CSF1 & $(0,0.1)$ & $0.1,0.3)$ & $(0.2,0.5)$ & $(0.1,0.3)$ & $(0.2,0.5)$ & $(0.2,0.5)$ & $(0.4,0.7)$ & $(0.2,0.5)$ & $(0.2,0.5)$ & $(0.2,0.5)$ & $(0.2,0.5)$ & $(0.4,0.7)$ & $(0.4,0.7)$ & $(0.4,0.7)$ & $(0.2,0.5)$ \\
\hline CSF2 & $.1,0.3)$ & $(0,0.1)$ & $.2,0.5)$ & $.2,0.5)$ & $(0.4,0.7)$ & $(0.2,0.5)$ & $(0.2,0.5)$ & $(0.2,0.5)$ & $(0.1,0.3)$ & $(0.2,0.5)$ & $(0.4,0.7)$ & $(0.4,0.7)$ & $(0.4,0.7)$ & $(0.2,0.5)$ & $0.4,0.7)$ \\
\hline CSF3 & $.1,0.3)$ & $1,0.3)$ & $(0,0.1)$ & $0.2,0.5)$ & $(0.2,0.5)$ & $(0.2,0.5)$ & $(0.2,0.5)$ & $(0.2,0.5)$ & $(0.1,0.3)$ & $(0.1,0.3)$ & $(0.4,0.7)$ & $(0.1,0.3)$ & $(0.4,0.7)$ & $(0.2,0.5)$ & $(0.4,0.7)$ \\
\hline CSF4 & $(0.1,0.3)$ & $(0.1,0.3)$ & $(0.2,0.5)$ & $(0,0.1)$ & $(0.2,0.5)$ & $(0.2,0.5)$ & $(0.4,0.7)$ & $(0.1,0.3)$ & $(0.4,0.7)$ & $(0.1,0.3)$ & $(0.4,0.7)$ & $(0.1,0.3)$ & $(0.2,0.5)$ & $(0.4,0.7)$ & $(0.2,0.5)$ \\
\hline CSF5 & $(0.1,0.3)$ & $(0.2,0.5)$ & $(0.2,0.5)$ & $(0.1,0.3)$ & $(0,0.1)$ & $(0.2,0.5)$ & $(0.2,0.5)$ & $(0.1,0.3)$ & $(0.2,0.5)$ & $(0.1,0.3)$ & $(0.4,0.7)$ & $(0.1,0.3)$ & $(0.4,0.7)$ & $(0.2,0.5)$ & $(0.4,0.7)$ \\
\hline CSF6 & $(0.2,0.5)$ & $(0.1,0.3)$ & $(0.1,0.3)$ & $(0.2,0.5)$ & $(0.2,0.5)$ & $(0,0.1)$ & $(0.2,0.5)$ & $(0.2,0.5)$ & $(0.2,0.5)$ & $(0.2,0.5)$ & $(0.4,0.7)$ & $(0.2,0.5)$ & $(0.4,0.7)$ & $(0.4,0.7)$ & $(0.4,0.7)$ \\
\hline CSF7 & $(0.2,0.5)$ & $(0.1,0.3)$ & $(0.2,0.5)$ & $(0.2,0.5)$ & $(0.2,0.5)$ & $(0.1,0.3)$ & $(0,0.1)$ & $(0.1,0.3)$ & $(0.2,0.5)$ & $(0.1,0.3)$ & $(0.6,0.9)$ & $(0.1,0.3)$ & $(0.4,0.7)$ & $(0.4,0.7)$ & $(0.4,0.7)$ \\
\hline CSF8 & $(0.1,0.3)$ & $(0.1,0.3)$ & $(0.1,0.3)$ & $(0.1,0.3)$ & $(0.2,0.5)$ & $(0.2,0.5)$ & $(0.4,0.7)$ & $(0,0.1)$ & $(0.1,0.3)$ & $(0.4,0.7)$ & $(0.4,0.7)$ & $(0.2,0.5)$ & $(0.4,0.7)$ & $(0.4,0.7)$ & $(0.4,0.7)$ \\
\hline CSF9 & $(0.1,0.3)$ & $(0.1,0.3)$ & $(0.2,0.5)$ & $(0.1,0.3)$ & $(0.1,0.3)$ & $(0.2,0.5)$ & $(0.1,0.3)$ & $(0.1,0.3)$ & $(0,0.1)$ & $(0.4,0.7)$ & $(0.4,0.7)$ & $(0.1,0.3)$ & $(0.4,0.7)$ & $(0.4,0.7)$ & $(0.4,0.7)$ \\
\hline CSF10 & $(0.1,0.3)$ & $(0.1,0.3)$ & $(0.2,0.5)$ & $(0.2,0.5)$ & $(0.2,0.5)$ & $(0.1,0.3)$ & $(0.2,0.5)$ & $(0.2,0.5)$ & $(0.4,0.7)$ & $(0,0.1)$ & $(0.4,0.7)$ & $(0.1,0.3)$ & $(0.4,0.7)$ & $(0.2,0.5)$ & $(0.6,0.9)$ \\
\hline CSF11 & $(0,0.1)$ & $(0.1,0.3)$ & $(0.1,0.3)$ & $(0.2,0.5)$ & $(0.2,0.5)$ & $(0.1,0.3)$ & $(0.2,0.5)$ & $(0.2,0.5)$ & $(0.1,0.3)$ & $(0.2,0.5)$ & $(0,0.1)$ & $(0.2,0.5)$ & $(0.4,0.7)$ & $(0.4,0.7)$ & $(0.4,0.7)$ \\
\hline CSF12 & $(0.2,0.5)$ & $(0.2,0.5)$ & $(0.1,0.3)$ & $(0.1,0.3)$ & $(0,0.1)$ & $(0.2,0.5)$ & $(0,0.1)$ & $(0.2,0.5)$ & $(0.1,0.3)$ & $(0.2,0.5)$ & $(0.6,0.9)$ & $(0,0.1)$ & $(0.6,0.9)$ & $(0.6,0.9)$ & $(0.4,0.7)$ \\
\hline CSF13 & $(0,0.1)$ & $(0.1,0.3)$ & $(0.1,0.3)$ & $(0.1,0.3)$ & $(0.2,0.5)$ & $(0.1,0.3)$ & $(0.2,0.5)$ & $(0.1,0.3)$ & $(0.2,0.5)$ & $(0.1,0.3)$ & $(0.2,0.5)$ & $(0.1,0.3)$ & $(0,0.1)$ & $(0.6,0.9)$ & $(0.6,0.9)$ \\
\hline CSF14 & $(0.1,0.3)$ & $(0.2,0.5)$ & $(0.1,0.3)$ & $(0.1,0.3)$ & $(0.1,0.3)$ & $(0.1,0.3)$ & $(0.1,0.3)$ & $(0.2,0.5)$ & $(0.1,0.3)$ & $(0.1,0.3)$ & $(0.2,0.5)$ & $(0.1,0.3)$ & $(0.4,0.7)$ & $(0,0.1)$ & $(0.4,0.7)$ \\
\hline CSF15 & $(0.1,0.3)$ & $(0.1,0.3)$ & $(0.1,0.3)$ & $(0.2,0.5)$ & $(0.1,0.3)$ & $(0.1,0.3)$ & $(0.2,0.5)$ & $(0,0.1)$ & $(0.2,0.5)$ & $(0.1,0.3)$ & $(0.2,0.5)$ & $(0.1,0.3)$ & $(0.4,0.7)$ & $(0.6,0.9)$ & $(0,0.1)$ \\
\hline
\end{tabular}

The level of influence of factor $\mathrm{x}$ the over the factor $\mathrm{y}$ is represented as Grey value $\left(\underline{\otimes} A_{x y}^{l}, \bar{\otimes} A_{x y}^{l}\right)$

Table 5: Average Grey relationship matrix for CSFs for effective adoption of sustainability initiatives in the automotive industry supply chain

\begin{tabular}{|c|c|c|c|c|c|c|c|c|c|c|c|c|c|c|c|}
\hline CSFs & CSF1 & $\begin{array}{l}\text { CSF2 } \\
\text {. }\end{array}$ & $\begin{array}{l}\text { CSF3 } \\
\end{array}$ & CSF4 & $\begin{array}{l}\text { CSF5 } \\
\end{array}$ & CSF6 & CSF7 & CSF8 & CSF9 & CSF10 & CSF11 & CSF12 & CSF13 & CSF14 & CSF15 \\
\hline CSF1 & $(0,0.1)$ & $(0.188,0.475)$ & $(0.35,0.65)$ & $(0.188,0.475)$ & $(0.2,0.5)$ & $(0.2,0.5)$ & $(0.375,0.675)$ & $(0.2,0.5)$ & $(0.2,0.5)$ & $(0.2,0.5)$ & $(0.35,0.65)$ & $(0.35,0.65)$ & $(0.275,0.575)$ & $(0.275,0.575)$ & $(0.225,0.525)$ \\
\hline $\begin{array}{l}\text { CSF2 } \\
\end{array}$ & $(0.188,0.475)$ & $(0,0.1)$ & $(0.35,0.65)$ & $(0.2,0.5)$ & $(0.375,0.675)$ & $(0.2,0.5)$ & $(0.2,0.5)$ & $(0.2,0.5)$ & $(0.1,0.3)$ & $(0.2,0.5)$ & $(0.3,0.6)$ & $(0.35,0.65)$ & $(0.375,0.675)$ & $(0.2,0.5)$ & $(0.375,0.675)$ \\
\hline CSF3 & $(0.188,0.475)$ & $(0.188,0.475)$ & $(0,0.1)$ & $(0.2,0.5)$ & $(0.2,0.5)$ & $(0.2,0.5)$ & $(0.2,0.5)$ & $(0.2,0.5)$ & $(0.1,0.3)$ & $(0.1,0.3)$ & $(0.4,0.7)$ & $(0.2,0.5)$ & $(0.4,0.7)$ & $(0.2,0.5)$ & $(0.4,0.7)$ \\
\hline CSF4 & $(0.1,0.3)$ & $(0.1,0.3)$ & $(0.2,0.5)$ & $(0,0.1)$ & $(0.2,0.5)$ & $(0.2,0.5)$ & $(0.4,0.7)$ & $(0.1,0.3)$ & $(0.4,0.7)$ & $(0.1,0.3)$ & $(0.4,0.7)$ & $(0.1,0.3)$ & $(0.2,0.5)$ & $(0.4,0.7)$ & $(0.2,0.5)$ \\
\hline CSF5 & $(0.1,0.3)$ & $(0.2,0.5)$ & $(0.2,0.5)$ & $(0.1,0.3)$ & $(0,0.1)$ & $(0.2,0.5)$ & $(0.2,0.5)$ & $(0.1,0.3)$ & $(0.2,0.5)$ & $(0.1,0.3)$ & $(0.4,0.7)$ & $(0.1,0.3)$ & $(0.4,0.7)$ & $(0.2,0.5)$ & $(0.4,0.7)$ \\
\hline CSF6 & $(0.2,0.5)$ & $(0.1,0.3)$ & $(0.1,0.3)$ & $(0.2,0.5)$ & $(0.2,0.5)$ & $(0,0.1)$ & $(0.2,0.5)$ & $(0.2,0.5)$ & $(0.2,0.5)$ & $(0.2,0.5)$ & $(0.525,0.825)$ & $(0.2,0.5)$ & $(0.575,0.875)$ & $(0.4,0.7)$ & $(0.575,0.875)$ \\
\hline CSF7 & $(0.2,0.5)$ & $(0.1,0.3)$ & $(0.2,0.5)$ & $(0.2,0.5)$ & $(0.2,0.5)$ & $(0.1,0.3)$ & $(0,0.1)$ & $(0.2,0.5)$ & $(0.2,0.5)$ & $(0.1,0.3)$ & $(0.55,0.85)$ & $(0.1,0.3)$ & $(0.4,0.7)$ & $(0.4,0.7)$ & $(0.4,0.7)$ \\
\hline CSF8 & $(0.1,0.3)$ & $(0.1,0.3)$ & $(0.1,0.3)$ & $(0.1,0.3)$ & $(0.2,0.5)$ & $(0.2,0.5)$ & $(0.4,0.7)$ & $(0,0.1)$ & $(0.125,0.35)$ & $(0.4,0.7)$ & $(0.575,0.875)$ & $(0.2,0.5)$ & $(0.575,0.875)$ & $(0.4,0.7)$ & $(0.4,0.7)$ \\
\hline CSF9 & $(0.125,0.35)$ & $(0.125,0.35)$ & $(0.2,0.5)$ & $(0.1,0.3)$ & $(0.1,0.3)$ & $(0.2,0.5)$ & $(0.1,0.3)$ & $(0.125,0.35)$ & $(0,0.1)$ & $(0.525,0.825)$ & $(0.4,0.7)$ & $(0.1,0.3)$ & $(0.575,0.875)$ & $(0.575,0.875)$ & $(0.4,0.7)$ \\
\hline CSF10 & $(0.113,0.325)$ & $(0.138,0.375)$ & $(0.2,0.5)$ & $(0.2,0.5)$ & $(0.2,0.5)$ & $(0.1,0.3)$ & $(0.2,0.5)$ & $(0.2,0.5)$ & $(0.5,0.8)$ & $(0,0.1)$ & $(0.4,0.7)$ & $(0.1,0.3)$ & $(0.4,0.7)$ & $(0.2,0.5)$ & $(0.6,0.9)$ \\
\hline CSF11 & $(0,0.1)$ & $(0.1,0.3)$ & $(0.1,0.3)$ & $(0.2,0.5)$ & $(0.2,0.5)$ & $(0.1,0.3)$ & $(0.2,0.5)$ & $(0.2,0.5)$ & $(0.1,0.3)$ & $(0.2,0.5)$ & $\begin{array}{l}(0,0.1) \\
\end{array}$ & $(0.2,0.5)$ & $\begin{array}{l}(0.5,0.85) \\
\end{array}$ & $(0.4,0.7)$ & $(0.4,0.7)$ \\
\hline CSF12 & $(0.2,0.5)$ & $(0.2,0.5)$ & $(0.1,0.3)$ & $(0.1,0.3)$ & $(0,0.1)$ & $(0.2,0.5)$ & $(0.1,0.3)$ & $(0.2,0.5)$ & $(0.1,0.3)$ & $(0.2,0.5)$ & $(0.575,0.875)$ & $(0,0.1)$ & $(0.6,0.9)$ & $(0.575,0.875)$ & $(0.5,0.8)$ \\
\hline CSF13 & $(0,0.1)$ & $(0.1,0.3)$ & $(0.1,0.3)$ & $(0.1,0.3)$ & $(0.2,0.5)$ & $\begin{array}{l}(0.1,0.3) \\
\end{array}$ & $(0.2,0.5)$ & $(0.1,0.3)$ & $(0.2,0.5)$ & $(0.1,0.3)$ & $(0.25,0.55)$ & $(0.1,0.3)$ & $(0,0.1)$ & $(0.45,0.75)$ & $\begin{array}{l}(0.375,675) \\
\end{array}$ \\
\hline CSF14 & $(0.1,0.3)$ & $(0.188,0.45)$ & $(0.1,0.3)$ & $(0.1,0.3)$ & $(0.1,0.3)$ & $(0.1,0.3)$ & $(0.1,0.3)$ & $(0.2,0.5)$ & $(0.1,0.3)$ & $(0.1,0.3)$ & $(0.25,0.55)$ & $(0.1,0.3)$ & $(0.4,0.7)$ & $(0,0.1)$ & $(0.35,0.65)$ \\
\hline$\overline{C S F 15}$ & $(0.088,0.25)$ & $(0.1,0.3)$ & $(0.1,0.3)$ & $(0.2,0.5)$ & $(0.1,0.3)$ & $(0.1,0.3)$ & $(0.2,0.5)$ & $(0,0.1)$ & $(0.2,0.5)$ & $(0.1,0.3)$ & $(0.275,0.575)$ & $(0.1,0.3)$ & $(0.325,0.625)$ & $(0.4,0.7)$ & $(0,0.1)$ \\
\hline
\end{tabular}

The level of influence of driver $\mathrm{x}$ the over the driver $\mathrm{y}$ is represented as Grey value $\left(\underline{\otimes} A_{x y}^{l}, \bar{\otimes} A_{x y}^{l}\right)$ 
Table 6: Normalised crisp relationship matrix for CSFs for effective adoption of sustainability initiatives in the automotive industry supply chain

\begin{tabular}{|c|c|c|c|c|c|c|c|c|c|c|c|c|c|c|c|}
\hline CSFs & CSF1 & CSF2 & CSF3 & CSF4 & CSF5 & CSF6 & CSF7 & CSF8 & CSF9 & CSF10 & CSF11 & CSF12 & CSF13 & CSF14 & CSF15 \\
\hline CSF1 & 0.0000 & 0.5795 & .7376 & 0.5795 & 0.4317 & 0.6286 & 0.7183 & 0.6286 & 0.3500 & 0.3370 & 0.5169 & 0.7376 & 0.4015 & 0.4153 & 0.3359 \\
\hline CSF2 & 5795 & 0.0000 & 7376 & 0.6286 & 0.7503 & 0.6286 & 0.4127 & 0.6286 & 0.1528 & 0.3370 & 0.4492 & 0.7376 & 0.5328 & 0.3136 & 0.5328 \\
\hline CSF3 & 0.5795 & 0.5795 & 0.0000 & 0.6286 & 0.4317 & 0.6286 & 0.4127 & 0.6286 & 0.1528 & 0.1474 & 0.5847 & 0.4525 & 0.5657 & 0.3136 & 0.5657 \\
\hline CSF4 & 0.2667 & 0.2667 & 0.4525 & 0.0000 & 0.4317 & 0.6286 & 0.7619 & 0.2667 & 0.6500 & 0.1474 & 0.5847 & 0.1949 & 0.3030 & 0.5847 & 0.3030 \\
\hline CSF5 & 0.2667 & 0.6286 & 0.4525 & 0.2667 & 0.0000 & 0.6286 & 0.4127 & 0.2667 & 0.3500 & 0.1474 & 0.5847 & 0.1949 & 0.5657 & 0.3136 & 0.5657 \\
\hline CSF6 & 0.6286 & 0.2667 & 0.1949 & 0.6286 & 0.4317 & 0.0000 & 0.4127 & 0.6286 & 0.3500 & 0.3370 & 0.7542 & 0.4525 & 0.7955 & 0.5847 & 0.7955 \\
\hline CSF7 & 0.6286 & 0.2667 & 0.4525 & 0.6286 & 0.4317 & 0.2667 & 0.0000 & 0.2667 & 0.3500 & $\begin{array}{l}0.1474 \\
\end{array}$ & 0.7880 & 0.1949 & 0.5657 & 0.5847 & 0.5657 \\
\hline CSF8 & 0.2667 & 0.2667 & 0.1949 & 0.2667 & 0.4317 & 0.6286 & 0.7619 & 0.0000 & 0.1985 & 0.6268 & 0.8219 & 0.4525 & 0.7955 & 0.5847 & 0.5657 \\
\hline CSF9 & 0.3500 & 0.3500 & 0.4525 & 0.2667 & 0.1864 & 0.6286 & 0.1786 & 0.3500 & 0.0000 & 0.8078 & 0.5847 & 0.1949 & 0.7955 & 0.8219 & 0.5657 \\
\hline CSF10 & 0.3077 & 0.3936 & 0.4525 & 0.6286 & 0.4317 & 0.2667 & 0.4127 & 0.6286 & 0.8000 & 0.0000 & 0.5847 & 0.1949 & 0.5657 & 0.3136 & 0.8283 \\
\hline CSF11 & 0.0000 & 0.2667 & 0.1949 & 0.6286 & 0.4317 & 0.2667 & 0.4127 & 0.6286 & 0.1528 & 0.3370 & 0.0000 & 0.4525 & 0.7367 & 0.5847 & 0.5657 \\
\hline CSF12 & 0.6286 & 0.6286 & 0.1949 & 0.2667 & 0.0000 & 0.6286 & 0.0000 & 0.6286 & 0.1528 & 0.3370 & 0.8219 & 0.0000 & 0.8283 & 0.8219 & 0.6970 \\
\hline CSF13 & 0.0000 & 0.2667 & 0.1949 & 0.2667 & 0.4317 & 0.2667 & 0.4127 & 0.2667 & 0.3500 & 0.1474 & 0.3814 & 0.1949 & 0.0000 & 0.6525 & 0.5328 \\
\hline CSF14 & 0.2667 & 0.5467 & 0.1949 & 0.2667 & $\begin{array}{l}0.1864 \\
\end{array}$ & 0.2667 & 0.1786 & 0.6286 & 0.1528 & 0.1474 & 0.3814 & 0.1949 & 0.5657 & 0.0000 & 0.5000 \\
\hline CSF15 & 0.2083 & 0.2667 & 0.1949 & 0.6286 & 0.1864 & 0.2667 & 0.4127 & 0.0000 & 0.3500 & 0.1474 & 0.4153 & 0.1949 & 0.4672 & 0.5847 & 0.0000 \\
\hline
\end{tabular}

Table 7: Final crisp relationship matrix for CSFs for effective adoption of sustainability initiatives in the automotive industry supply chain

\begin{tabular}{|c|c|c|c|c|c|c|c|c|c|c|c|c|c|c|c|}
\hline CSFs & SF1 & CSF2 & CSF3 & CSF4 & CSF5 & CSF6 & CSF7 & CSF8 & CSF9 & CSF10 & CSF11 & CSF12 & CSF13 & CSF14 & CSF15 \\
\hline CSF1 & 0000 & 0.2898 & 4794 & 0.2898 & 0.2914 & 0.3143 & 0.5028 & 0.3143 & 0.2800 & 0.2780 & 0.4523 & 0.4794 & 0.3614 & 0.3634 & 0.3023 \\
\hline CSF2 & 2898 & 0000 & 0.4794 & 0.3143 & 0.5064 & 0.3143 & 0.2889 & 0.3143 & 0.1222 & 0.2780 & 0.3930 & 0.4794 & 0.4795 & 0.2744 & 0.4795 \\
\hline CSF3 & 2898 & 0.2898 & 0.0000 & 0.3143 & 0.2914 & 0.3143 & 0.2889 & 0.3143 & 0.122 & 0.1216 & 0.5116 & 0.2941 & 0.5091 & 0.2744 & 0.5091 \\
\hline CSF4 & 1333 & 0.1333 & 0.2941 & 0.0000 & 0.2914 & 0.3143 & 0.5333 & 0.1333 & 0.5200 & 0.1216 & 0.5116 & 0.1267 & 0.2727 & 0.5116 & 0.2727 \\
\hline CSF5 & 1333 & 0.3143 & 0.2941 & 0.1333 & 0.0000 & 0.3143 & 0.2889 & 0.1333 & 0.2800 & 0.1216 & 0.5116 & 0.1267 & 0.5091 & 0.2744 & 0.5091 \\
\hline CSF6 & 0.3143 & 0.1333 & 0.1267 & 0.3143 & 0.2914 & 0.0000 & 0.2889 & 0.3143 & 0.2800 & 0.2780 & 0.6599 & 0.2941 & 0.7159 & 0.5116 & 0.7159 \\
\hline CSF7 & 3143 & 0.1333 & 0.2941 & 0.3143 & 0.2914 & 1333 & 0.0000 & 0.1333 & 0.2800 & 0.1216 & 0.6895 & 0.1267 & 0.5091 & 0.5116 & 0.5091 \\
\hline CSF8 & 1333 & 0.1333 & 0.1267 & 0.1333 & 0.2914 & 0.3143 & 0.5333 & 0.0000 & 0.1588 & 0.5171 & 0.7192 & 0.2941 & 0.7159 & 0.5116 & 0.5091 \\
\hline CSF9 & 0.1750 & 0.1750 & 0.2941 & 0.1333 & 0.1258 & 0.3143 & 0.1250 & $\begin{array}{l}0.1750 \\
\end{array}$ & 0.0000 & 0.6665 & 0.5116 & 0.1267 & 0.7159 & 0.7192 & 0.5091 \\
\hline CSF10 & 0.1538 & 0.1968 & 0.2941 & 0.3143 & 0.2914 & 0.1333 & 0.2889 & 0.3143 & 0.6400 & 0.0000 & 0.5116 & 0.1267 & 0.5091 & 0.2744 & 0.7455 \\
\hline CSF11 & 0.0000 & 0.1333 & 0.1267 & 0.3143 & 0.2914 & 0.1333 & 0.2889 & 0.3143 & 0.1222 & 0.2780 & 0.0000 & 0.2941 & 0.6630 & 0.5116 & 0.5091 \\
\hline CSF12 & 0.3143 & 0.3143 & 0.1267 & 0.1333 & 0.0000 & 0.3143 & 0.0000 & 0.3143 & 0.1222 & 0.2780 & 0.7192 & 0.0000 & 0.7455 & 0.7192 & 0.6273 \\
\hline CSF13 & 0.0000 & 0.1333 & 0.1267 & 0.1333 & 0.2914 & 0.1333 & 0.2889 & 0.1333 & 0.2800 & 0.1216 & 0.3337 & 0.1267 & 0.0000 & 0.5709 & 0.4795 \\
\hline CSF14 & 0.1333 & 0.2733 & 0.1267 & 0.1333 & 0.1258 & 0.1333 & 0.1250 & 0.3143 & 0.1222 & 0.1216 & 0.3337 & 0.1267 & 0.5091 & 0.0000 & 0.4500 \\
\hline CSF15 & 0.1042 & 0.1333 & 0.1267 & 0.3143 & 0.1258 & 0.1333 & 0.2889 & 0.0000 & 0.2800 & 0.1216 & 0.3634 & 0.1267 & 0.4205 & 0.5116 & 0.0000 \\
\hline
\end{tabular}


Table 8: Normalised direct relationship matrix for CSFs for effective adoption of sustainability initiatives in the automotive industry supply chain

\begin{tabular}{|c|c|c|c|c|c|c|c|c|c|c|c|c|c|c|c|}
\hline CSFs & CSF1 & CSF2 & CSF3 & CSF4 & CSF5 & CSF6 & CSF7 & CSF8 & CSF9 & CSF10 & CSF11 & CSF12 & CSF13 & CSF14 & CSF15 \\
\hline CSF1 & 0.0000 & 0.0379 & 0.0628 & 0.0379 & 0.0382 & 0.0412 & 0.0658 & 0.0412 & 0.0367 & 0.0364 & 0.0592 & 0.0628 & 0.0473 & 0.0476 & 0.0396 \\
\hline CSF 2 & 0379 & 0.0000 & 0.0628 & 0.0412 & 0.0663 & 0.0412 & 0.0378 & 0.0412 & 0.0160 & 0.0364 & 0.0515 & 0.0628 & 0.0628 & 0.0359 & 0.0628 \\
\hline CSF 3 & $\overline{0379}$ & 0.0379 & 0.0000 & 0.0412 & 0.0382 & 0.0412 & 0.0378 & 0.0412 & 0.0160 & 0.0159 & 0.0670 & 0.0385 & 0.0667 & 0.0359 & 0.0667 \\
\hline CSF4 & 0.0175 & 0.0175 & 0.0385 & 0.0000 & 0.0382 & 0.0412 & 0.0698 & 0.0175 & 0.0681 & 0.0159 & 0.0670 & 0.0166 & 0.0357 & 0.0670 & 0.0357 \\
\hline CSF5 & 0.0175 & 0.0412 & 0.0385 & 0.0175 & 0.0000 & 0.0412 & 0.0378 & 0.0175 & 0.0367 & 0.0159 & 0.0670 & 0.0166 & 0.0667 & 0.0359 & 0.0667 \\
\hline CSF6 & 0.0412 & 0.0175 & 0.0166 & 0.0412 & 0.0382 & 0.0000 & $\begin{array}{l}0.0378 \\
\end{array}$ & 0.0412 & 0.0367 & 0.0364 & $\begin{array}{l}0.0864 \\
\end{array}$ & 0.0385 & 0.0938 & 0.0670 & 0.0938 \\
\hline CSF7 & 0.0412 & 0.0175 & 0.0385 & 0.0412 & 0.0382 & 0.0175 & 0.0000 & 0.0175 & 0.0367 & 0.0159 & 0.0903 & 0.0166 & 0.0667 & 0.0670 & 0.0667 \\
\hline CSF8 & 0.0175 & 0.0175 & 0.0166 & 0.0175 & 0.0382 & 0.0412 & 0.0698 & 0.0000 & 0.0208 & 0.0677 & 0.0942 & 0.0385 & 0.0938 & 0.0670 & 0.0667 \\
\hline CSF9 & 0.0229 & 0.0229 & 0.0385 & 0.0175 & 0.0165 & 0.0412 & 0.0164 & 0.0229 & 0.0000 & 0.0873 & 0.0670 & 0.0166 & 0.0938 & 0.0942 & 0.0667 \\
\hline CSF10 & 0.0201 & 0.0258 & 0.0385 & 0.0412 & 0.0382 & 0.0175 & 0.0378 & 0.0412 & 0.0838 & 0.0000 & 0.0670 & 0.0166 & 0.0667 & 0.0359 & 0.0976 \\
\hline CSF11 & 0.0000 & 0.0175 & 0.0166 & 0.0412 & 0.0382 & 0.0175 & 0.0378 & 0.0412 & 0.0160 & 0.0364 & 0.0000 & 0.0385 & 0.0868 & 0.0670 & 0.0667 \\
\hline CSF12 & 0.0412 & 0.0412 & 0.0166 & 0.0175 & 0.0000 & 0.0412 & 0.0000 & 0.0412 & 0.0160 & 0.0364 & 0.0942 & 0.0000 & 0.0976 & 0.0942 & 0.0821 \\
\hline CSF13 & 0.0000 & 0.0175 & 0.0166 & 0.0175 & 0.0382 & 0.0175 & 0.0378 & 0.0175 & 0.0367 & 0.0159 & 0.0437 & 0.0166 & 0.0000 & 0.0748 & 0.0628 \\
\hline CSF14 & 0.0175 & 0.0358 & 0.0166 & 0.0175 & 0.0165 & 0.0175 & 0.0164 & 0.0412 & 0.0160 & 0.0159 & 0.0437 & 0.0166 & 0.0667 & 0.0000 & 0.0589 \\
\hline CSF15 & 0.0136 & 0.0175 & 0.0166 & 0.0412 & 0.0165 & 0.0175 & 0.0378 & 0.0000 & 0.0367 & 0.0159 & 0.0476 & 0.0166 & 0.0551 & 0.0670 & 0.0000 \\
\hline
\end{tabular}

Table 9: Total relationship matrix for CSFs for effective adoption of sustainability initiatives in the automotive industry supply chain

\begin{tabular}{|c|c|c|c|c|c|c|c|c|c|c|c|c|c|c|c|}
\hline CSFs & CSF1 & CSF2 & CSF3 & CSF4 & CSF5 & CSF6 & CSF7 & CSF8 & CSF9 & CSF10 & CSF11 & CSF12 & CSF13 & CSF14 & CSF15 \\
\hline CSF1 & 0.0301 & 0.0720 & 0.0990 & 0.0791 & 0.0807 & 0.0795 & 0.1134 & 0.0803 & 0.0798 & 0.0765 & 0.1444 & 0.0985 & 0.1410 & 0.1300 & 0.1275 \\
\hline CSF2 & 0.0656 & 0.0356 & 0.0985 & 0.0816 & 0.1067 & 0.0794 & 0.0878 & 0.0791 & 0.0608 & 0.0748 & 0.1359 & 0.0980 & 0.1535 & 0.1178 & 0.1478 \\
\hline CSF3 & 0.0612 & 0.0669 & 0.0337 & 0.0769 & 0.0758 & 0.0740 & 0.0826 & 0.0740 & 0.0551 & 0.0513 & 0.1390 & 0.0711 & 0.1458 & $\begin{array}{l}0.1091 \\
\end{array}$ & 0.1401 \\
\hline CSF4 & 0.0410 & 0.0458 & 0.0690 & 0.0348 & 0.0725 & 0.0715 & 0.1075 & 0.0499 & 0.1015 & 0.0507 & 0.1347 & 0.0465 & 0.1137 & 0.1339 & 0.1076 \\
\hline CSF5 & 0.0388 & 0.0661 & 0.0668 & 0.0506 & 0.0346 & 0.0693 & 0.0758 & 0.0474 & 0.0695 & 0.0474 & 0.1290 & 0.0459 & 0.1365 & 0.1003 & 0.1318 \\
\hline CSF6 & 0.0661 & 0.0518 & 0.0541 & 0.0818 & 0.0801 & 0.0381 & 0.0884 & 0.0785 & 0.0814 & 0.0762 & 0.1666 & 0.0741 & 0.1830 & 0.1501 & 0.1766 \\
\hline CSF7 & 0.0617 & 0.0466 & 0.0693 & 0.0752 & 0.0735 & 0.0495 & 0.0435 & 0.0502 & 0.0728 & 0.0496 & 0.1552 & 0.0479 & 0.1417 & 0.1346 & 0.1359 \\
\hline CSF8 & 0.0436 & 0.0507 & 0.0528 & 0.0591 & 0.0798 & 0.0755 & 0.1159 & 0.0387 & 0.0660 & 0.1038 & 0.1727 & 0.0725 & 0.1818 & 0.1472 & 0.1520 \\
\hline CSF9 & 0.0466 & 0.0542 & 0.0713 & 0.0563 & 0.0566 & 0.0733 & 0.0626 & 0.0597 & 0.0434 & 0.1200 & 0.1400 & 0.0499 & 0.1747 & 0.1652 & 0.1462 \\
\hline CSF10 & 0.0449 & 0.0567 & 0.0731 & 0.0789 & 0.0771 & 0.0536 & 0.0845 & 0.0746 & 0.1226 & 0.0404 & 0.1428 & 0.0502 & 0.1516 & 0.1153 & 0.1735 \\
\hline CSF11 & 0.0211 & 0.0435 & 0.0443 & 0.0707 & 0.0694 & 0.0461 & 0.0753 & 0.0684 & 0.0514 & 0.0650 & 0.0654 & 0.0636 & 0.1533 & 0.1289 & 0.1308 \\
\hline CSF12 & 0.0629 & 0.0708 & 0.0496 & 0.0556 & 0.0407 & 0.0733 & 0.0475 & 0.0766 & 0.0557 & 0.0723 & 0.1636 & 0.0352 & 0.1777 & 0.1659 & 0.1577 \\
\hline CSF13 & 0.0173 & 0.0385 & 0.0390 & 0.0425 & 0.0623 & 0.0401 & 0.0662 & 0.0405 & 0.0622 & 0.0404 & 0.0933 & 0.0377 & 0.0582 & 0.1224 & 0.1133 \\
\hline CSF14 & 0.0330 & 0.0547 & 0.0389 & 0.0424 & 0.0432 & 0.0402 & 0.0477 & 0.0626 & 0.0424 & 0.0405 & 0.0924 & 0.0393 & 0.1194 & 0.0509 & 0.1084 \\
\hline CSF15 & 0.0297 & 0.0377 & 0.0392 & 0.0643 & 0.0420 & 0.0396 & 0.0660 & 0.0241 & 0.0624 & 0.0395 & 0.0952 & 0.0376 & 0.1078 & 0.1145 & 0.0519 \\
\hline
\end{tabular}


Table 10: Cause/effect parameters for CSFs for effective adoption of sustainability initiatives in the automotive industry supply chain

\begin{tabular}{|c|c|c|c|c|c|}
\hline CSFs for effective adoption of sustainability initiatives in supply chains & $\mathbf{R}$ & D & $\mathbf{R}+\mathbf{D}$ & R-D & Cause/Effect \\
\hline CSF1 & 1.4318 & 0.6635 & 2.0953 & 0.7682 & Cause \\
\hline CSF 2 & 1.4228 & 0.7916 & 2.2145 & 0.6312 & Cause \\
\hline CSF 3 & 1.2568 & 0.8986 & 2.1554 & 0.3583 & Cause \\
\hline CSF4 & 1.1807 & 0.9498 & 2.1304 & 0.2309 & Cause \\
\hline CSF5 & 1.1097 & 0.9951 & 2.1048 & 0.1146 & Cause \\
\hline CSF6 & 1.4469 & 0.9028 & 2.3496 & 0.5441 & Cause \\
\hline CSF7 & 1.2072 & 1.1647 & 2.3719 & 0.0425 & Cause \\
\hline CSF8 & 1.4122 & 0.9048 & 2.3170 & 0.5074 & Cause \\
\hline CSF9 & 1.3201 & 1.0269 & 2.3470 & 0.2932 & Cause \\
\hline CSF10 & 1.3398 & 0.9485 & 2.2883 & 0.3913 & Cause \\
\hline CSF11 & 1.0972 & 1.9701 & 3.0673 & -0.8729 & Effect \\
\hline CSF12 & 1.3051 & 0.8682 & 2.1732 & 0.4369 & Cause \\
\hline$\sqrt{20}$ & 0.8740 & 2.1397 & 3.0137 & -1.2657 & Effect \\
\hline 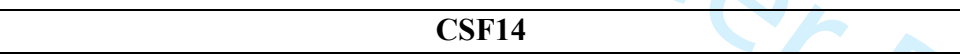 & 0.8560 & 1.8861 & 2.7420 & -1.0301 & Effect \\
\hline CSF15 & 0.8516 & 2.0015 & 2.8530 & -1.1499 & Effect \\
\hline
\end{tabular}


Table 11: Weights assigned for eight experts during sensitivity analysis

\begin{tabular}{l|c|c|c|c|c|c|c|c}
\hline Run & Expert 1 & Expert 2 & Expert 3 & Expert 4 & Expert 5 & Expert 6 & Expert 7 & Expert 8 \\
\hline Sensitivity Run 1 & 0.3 & 0.1 & 0.1 & 0.1 & 0.1 & 0.1 & 0.1 & 0.1 \\
\hline Sensitivity Run 2 & 0.1 & 0.3 & 0.1 & 0.1 & 0.1 & 0.1 & 0.1 & 0.1 \\
\hline Sensitivity Run 3 & 0.1 & 0.1 & 0.3 & 0.1 & 0.1 & 0.1 & 0.1 & 0.1 \\
\hline Sensitivity Run 4 & 0.1 & 0.1 & 0.1 & 0.3 & 0.1 & 0.1 & 0.1 & 0.1 \\
\hline Sensitivity Run 5 & 0.1 & 0.1 & 0.1 & 0.1 & 0.3 & 0.1 & 0.1 & 0.1 \\
\hline Sensitivity Run 6 & 0.1 & 0.1 & 0.1 & 0.1 & 0.1 & 0.3 & 0.1 & 0.1 \\
\hline Sensitivity Run 7 & 0.1 & 0.1 & 0.1 & 0.1 & 0.1 & 0.1 & 0.3 & 0.1 \\
\hline Sensitivity Run 8 & 0.1 & 0.1 & 0.1 & 0.1 & 0.1 & 0.1 & 0.1 & 0.3 \\
\hline
\end{tabular}

Table 12: Sensitivity analysis of CSFs for effective adoption of sustainability initiatives in the supply chains

\begin{tabular}{|c|c|c|c|c|c|c|c|c|c|c|c|c|}
\hline \multirow{2}{*}{ CSFs } & \multicolumn{3}{|c|}{ Sensitivity Run 1} & \multicolumn{3}{|c|}{ Sensitivity Run 2} & \multicolumn{3}{|c|}{ Sensitivity Run 3} & \multicolumn{3}{|c|}{ Sensitivity Run 4} \\
\hline & $\mathbf{R}+\mathbf{D}$ & R-D & Rank & $\mathbf{R}+\mathbf{D}$ & R-D & Rank & $\mathbf{R}+\mathbf{D}$ & R-D & Rank & $\mathbf{R}+\mathbf{D}$ & R-D & Rank \\
\hline CSF1 & 2.1084 & 0.7837 & 1 & 2.1243 & 0.7600 & 1 & 2.0794 & 0.7616 & 1 & 2.1378 & 0.7853 & 1 \\
\hline CSF 2 & 2.2459 & 6601 & 2 & 2.2164 & 0.6347 & 2 & 2.2052 & 0.6015 & 2 & 2.2658 & 0.6193 & 2 \\
\hline CSF 3 & 2.1783 & 0.3562 & 7 & 2.1473 & 0.3651 & 7 & 2.1424 & 0.3547 & 7 & 2.1981 & 0.3646 & 7 \\
\hline CSF4 & 2.1750 & 0.2455 & 9 & 2.1277 & 0.2289 & 9 & 3.0000 & 0.2278 & 9 & 2.1679 & 0.2331 & 9 \\
\hline CSF5 & 2.1557 & 0.1222 & 10 & 2.1010 & 0.1138 & 10 & 2.0881 & 0.1100 & 10 & 2.1410 & 0.1165 & 10 \\
\hline CSF6 & 2.3798 & 0.5329 & 3 & 2.3279 & 0.5234 & 3 & 2.3371 & 0.5465 & 3 & 2.3975 & 0.5612 & 3 \\
\hline CSF7 & 2.4343 & 0.0498 & 11 & 2.3485 & 0.0186 & 11 & 2.3460 & 0.0540 & 11 & 2.4160 & 0.0449 & 11 \\
\hline CSF8 & 2.3493 & 0.5023 & 4 & 2.3101 & 0.5069 & 4 & 2.2957 & 0.5064 & 4 & 2.3559 & 0.5186 & 4 \\
\hline CSF9 & 2.3661 & 0.2688 & 8 & 2.3557 & 0.3045 & 8 & 2.3376 & 0.3033 & 8 & 2.3798 & 0.2980 & 8 \\
\hline CSF 10 & 2.3293 & 0.3933 & 6 & 2.2992 & 0.4014 & 6 & 2.2829 & 0.3991 & 6 & 2.3230 & 0.3987 & 6 \\
\hline CSF11 & 3.1033 & -0.8645 & 12 & 3.0266 & -0.8490 & 12 & 3.0472 & -0.8853 & 12 & 3.1304 & -0.8910 & 12 \\
\hline CSF12 & 2.2262 & 0.4469 & 5 & 2.1708 & 0.4304 & 5 & 2.1425 & 0.4141 & 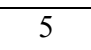 & 2.2224 & 0.4496 & 5 \\
\hline CSF13 & 3.0777 & -1.2472 & 15 & 2.9930 & -1.2796 & 15 & 3.0016 & -1.2539 & 15 & 3.0574 & -1.2676 & 15 \\
\hline CSF14 & 2.8396 & -1.0780 & 13 & 2.7551 & -1.0173 & 13 & 2.7149 & -0.9891 & 13 & 2.7906 & -1.0677 & 13 \\
\hline CSF15 & 2.9460 & -1.1722 & 14 & 2.8436 & -1.1418 & 14 & 2.8444 & -1.1507 & 14 & 2.9002 & -1.1633 & 14 \\
\hline \multirow{2}{*}{ CSFs } & \multicolumn{3}{|c|}{ Sensitivity Run 5} & \multicolumn{3}{|c|}{ Sensitivity Run 6} & \multicolumn{3}{|c|}{ Sensitivity Run 7} & \multicolumn{3}{|c|}{ Sensitivity Run 8} \\
\hline & $\mathbf{R}+\mathbf{D}$ & R-D & Rank & $\mathbf{R}+\mathbf{D}$ & R-D & Rank & $\mathbf{R}+\mathbf{D}$ & R-D & Rank & $\mathbf{R}+\mathbf{D}$ & R-D & Rank \\
\hline CSF1 & 2.0907 & 0.7547 & 1 & 2.0532 & 0.7427 & 4 & 2.0612 & 0.7756 & 1 & 2.0600 & 0.7489 & 1 \\
\hline CSF 2 & 2.2106 & 0.6237 & 2 & 2.1851 & 0.6255 & 2 & 2.1528 & 0.6228 & 2 & 2.1887 & 0.6351 & 2 \\
\hline CSF 3 & 2.1425 & 0.3540 & 1 & 2.1279 & 0.3526 & 7 & 2.1250 & 0.3496 & 7 & 2.1437 & 0.3510 & 7 \\
\hline CSF4 & 2.1126 & 0.2264 & 9 & 2.0935 & 0.2202 & S & 2.0982 & 0.2235 & 9 & 2.1139 & 0.2214 & 9 \\
\hline CSF5 & 2.0870 & 0.1123 & 10 & 2.0714 & 0.1097 & 10 & 2.0722 & 0.1097 & 10 & 2.0894 & 0.1075 & 10 \\
\hline CSF6 & 2.3364 & 0.5454 & 3 & 2.3180 & 0.5380 & 3 & 2.3170 & 0.5396 & 3 & 2.3347 & 0.5403 & 3 \\
\hline CSF7 & 2.3557 & 0.0423 & 11 & 2.3388 & 0.0389 & 11 & 2.3405 & 0.0398 & 11 & 2.3598 & 0.0358 & 11 \\
\hline CSF8 & 2.2959 & 0.5046 & 4 & 2.2923 & 0.4964 & 4 & 2.2764 & 0.4995 & 4 & 2.3119 & 0.5007 & 4 \\
\hline CSF9 & 2.3371 & 0.3022 & 8 & 2.2709 & 0.3086 & 8 & 2.3104 & 0.2865 & 8 & 2.3110 & 0.3258 & 8 \\
\hline CSF10 & 2.2824 & 0.3978 & 6 & 2.2030 & 0.3996 & 0 & 2.2543 & 0.3830 & 6 & 2.2540 & 0.3678 & 6 \\
\hline CSF11 & 3.0639 & -0.8819 & 12 & 3.0217 & -0.8580 & 12 & 3.0389 & -0.8779 & 12 & 3.0379 & -0.8609 & 12 \\
\hline CSF12 & 2.1559 & 0.4266 & 5 & 2.1505 & 0.4323 & 5 & 2.0927 & 0.4710 & 5 & 2.1210 & 0.4832 & 5 \\
\hline CSF13 & 3.0105 & -1.2632 & 15 & 2.9761 & -1.2692 & 15 & 2.9805 & -1.2484 & 15 & 2.9611 & -1.2851 & 15 \\
\hline CSF14 & 2.7136 & -1.0127 & 13 & 2.6846 & -1.0180 & 13 & 2.6822 & -1.0181 & 13 & 2.6992 & -1.0152 & 13 \\
\hline CSF15 & 2.8220 & -1.1321 & 14 & 2.8169 & -1.1194 & 14 & 2.7926 & -1.1560 & 14 & 2.8125 & -1.1564 & 14 \\
\hline
\end{tabular}

